\title{
42. CENOZOIC SILICOFLAGELLATES AND EBRIDIANS FROM ODP LEG 113: BIOSTRATIGRAPHY AND NOTES ON MORPHOLOGIC VARIABILITY ${ }^{1}$
}

\author{
Kevin McCartney ${ }^{2}$ and Sherwood W. Wise, $\mathrm{Jr}^{3}$
}

\begin{abstract}
Silicoflagellates are present at ODP 113 Sites $689,690,693,695,696$, and 697 . These are generally Neogene in age except for Oligocene and Eocene silicoflagellates and ebridians at Sites 689, 690, and 693, and Lower Cretaceous (Albian) silicoflagellates at Site 693 (the latter are described in McCartney, et al., this volume). The uppermost Miocenelowermost Pliocene of most sites contains an interesting assemblage of Distephanus speculum speculum that lack apical rings (the "pseudofibula plexus"). These co-occur with the youngest ice-rafted sediments deposited during the late Miocene-early Pliocene West Antarctic glaciations and are discussed in detail to show the variability within this group. We believe that these variations resulted from environmental stress, the precise nature of which is still undetermined. The "pseudofibulid" condition has developed at several different times during the Cenozoic within the Distephanus speculum lineage to produce such ecophenotypes. One new form from the "pseudofibula plexus," D. s. speculum f. pseudopentagonus, is described.
\end{abstract}

\section{INTRODUCTION}

Ocean Drilling Program Leg 113 was the first drilling leg to explore the Weddell Sea (Figs. 1, 2). This complemented the inaugural leg to the high latitudes of the Antarctic Ocean, Leg 28 to the Ross Sea, which recovered the first extensive sequences of silicoflagellates from this region. Most of the six other legs that followed to the Southern Ocean were to the subantarctic region (Table 1).

During Leg 113, silicoflagellates were recovered from the Neogene at eight sites, the Paleogene (Oligocene-Eocene) at three sites, and the Lower Cretaceous at one site (Table 2). They were commingled with carbonate pelagic sediments throughout most of Sites 689 and 690 on Maud Rise and with biosiliceous and siliciclastic sediments at the others. At all sites they were seldom abundant, except at certain intervals in the Pliocene and Pleistocene, and their diversities were also generally low. For the Maud Rise sites, low abundances and diversities can be attributed to low phytoplankton productivity, as indicated by the low sedimentation rates recorded at these sites (Barker, Kennett, et al., 1988). They were more abundant there, however, than at comparable water depths on the adjacent Antarctic margin (Site 693), where the section was considerably diluted by siliciclastics. The accumulation of biosiliceous material was high in the middle Miocene to Pliocene at most other nonabyssal sites, but drilling problems precluded recovery in some holes, particularly at Site 696. In general, abundances and diversities of silicoflagellates at the Leg 113 sites were far lower than at subantarctic sites to the north, which are located closer to zones of upwelling along boundaries between major water masses (present and past), such as the Polar Front Zone.

An interesting phenomenon noted in silicoflagellate assemblages from two intervals is exceptional intraspecific variability or morphologic plasticity. This was expressed consistently at all sites among the lower Pliocene representatives of Distephanus speculum speculum, an assemblage we refer to as the "pseudofi-

\footnotetext{
${ }^{1}$ Barker, P. F., Kennett, J. P., et al., 1990. Proc. ODP, Sci. Results, 113: College Station, TX (Ocean Drilling Program).

2 Department of Geology, Florida State University, Tallahassee, FL 32306; present address: University of Maine at Presque Isle, Presque Isle, ME 04769.

${ }^{3}$ Department of Geology, Florida State University, Tallahassee, FL 32306.
}

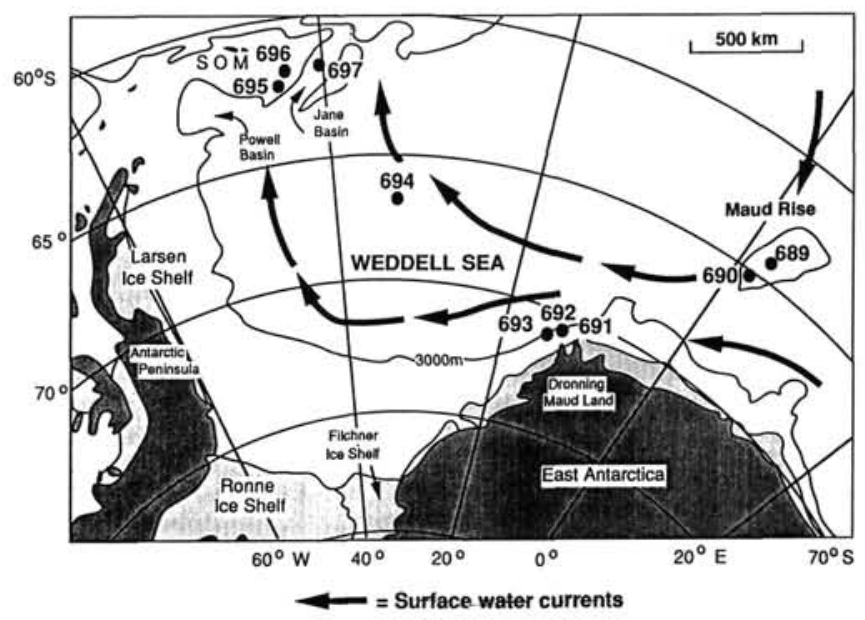

Figure 1. Location of sites drilled on ODP Leg 113; arrows indicate the surface current regimes prevalent during the late Neogene.

bula plexus." We speculate in this paper that the morphologic plasticity within this plexus might be attributable to environmental stress. Exceptional variability was also seen in a much older Lower Cretaceous (lower Albian) assemblage from Site 693 where the cause is more likely purely genetic. We discuss the Pliocene case in some detail in this paper and the Lower Cretaceous occurrence in a companion paper (McCartney et al., this volume).

In addition to silicoflagellates, we chronicle here occurrences of ebridians, which were locally abundant at Site 689 . Notable there is an assemblage near the Eocene/Oligocene boundary which contains exceptionally large and complicated forms previously known only from middle Eocene piston cores taken on the Falkland Plateau to the north.

\section{SAMPLE PREPARATION, METHODS OF STUDY, AND ZONATION}

For Holes 689B and 690B, in which there was an abundance of calcareous material, raw samples were placed in $100 \mathrm{~mL}$ beakers and a small amount of $30 \%$ hydrogen peroxide added. After 2-4 hr the beaker was placed in an ultrasonic cleaner and distilled water was added to approximately the $25 \mathrm{~mL}$ level. $\mathrm{HCl}$ 


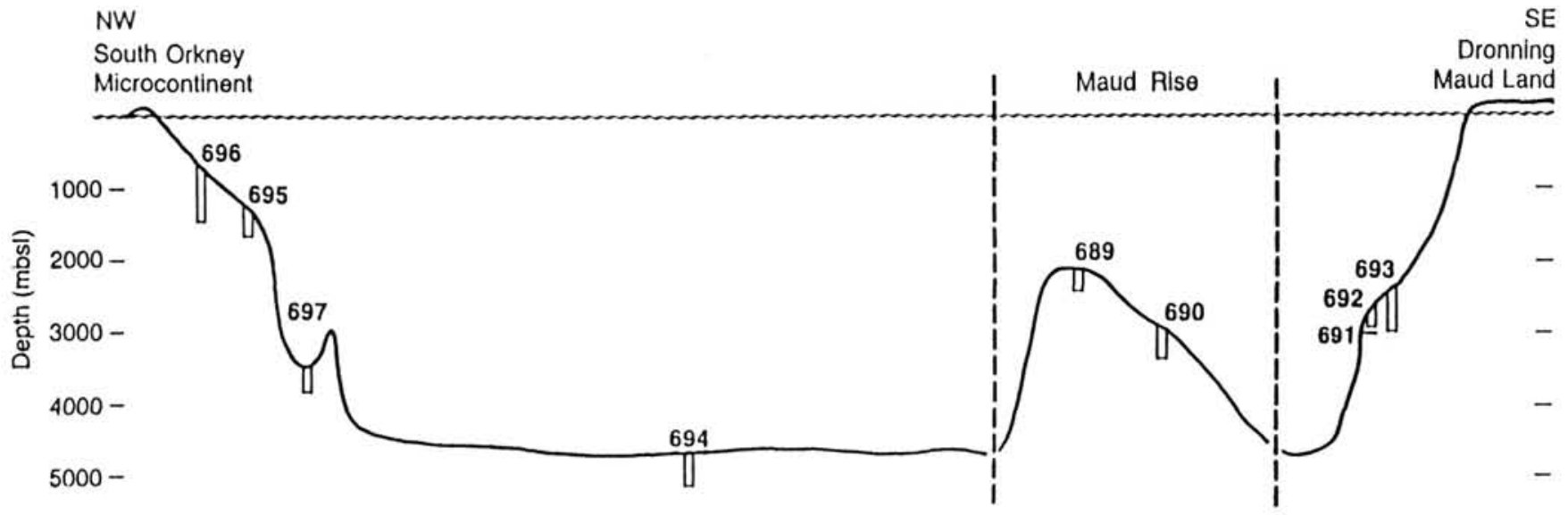

Figure 2. Cross section showing ocean bottom profile and water depth of holes drilled during Leg 113.

Table 1. Silicoflagellate and ebridian studies from southern ocean sites $\left(>45^{\circ} \mathrm{S}\right)$. All publications listed here, unless stated otherwise, include charts showing relative abundances. $X=$ present throughout the series; $U, M$, or $L=$ subseries.

\begin{tabular}{|c|c|c|c|c|c|c|c|c|}
\hline Leg (Sites) & Pleistocene & Pliocene & Miocene & Oligocene & Eocene & Paleocene & Pleistocene & Reference \\
\hline $28(265-271)$ & $x$ & $\mathrm{x}$ & $x$ & $\begin{array}{l}\mathrm{X} \\
\mathrm{x}\end{array}$ & & & & $\begin{array}{l}\text { Bukry (1975a) } \\
\text { Ciesielski (1975) }\end{array}$ \\
\hline $29(275,278,280)$ & $\mathrm{x}$ & $\mathrm{x}$ & $\mathrm{x}$ & $\mathrm{x}$ & $\mathrm{U}, \mathrm{M}$ & & $\begin{array}{l}\mathrm{U} \\
\mathrm{U} \\
\mathrm{U}\end{array}$ & $\begin{array}{l}\text { Bukry }(1975 \mathrm{c}) \\
\text { Hajos (1975) } \\
\text { Perch-Nielsen }(1975 \mathrm{a}, \mathrm{b})\end{array}$ \\
\hline $35(322-323)$ & (no abundar & $\begin{array}{c}\text { ce charts) } \\
X\end{array}$ & $\mathrm{x}$ & L & $\mathrm{U}$ & & & $\begin{array}{l}\text { Bukry (1976b) } \\
\text { Haq and Riley (1976) } \\
\text { Hajos (1976) }\end{array}$ \\
\hline $36(326-329)$ & $\begin{array}{l}x \\
x\end{array}$ & $\begin{array}{l}\mathrm{X} \\
\mathrm{U}\end{array}$ & $\begin{array}{l}\mathrm{U} \\
\mathrm{X}\end{array}$ & $\mathrm{x}$ & $U, L$ & $\stackrel{U}{U}$ & & $\begin{array}{l}\text { Bukry (1976a) } \\
\text { Busen and Wise (1977) }\end{array}$ \\
\hline $38(336-346)$ & & & $\mathrm{M}, \mathrm{L}$ & L & M?, U & & & Bukry (1976c) \\
\hline $71(511-514)$ & $\mathrm{x}$ & $\mathrm{x}$ & $\mathrm{x}$ & $\mathrm{x}$ & & & & Shaw and Ciesielski (1983) \\
\hline $90(594)$ & $\mathrm{x}$ & $\mathrm{x}$ & $\mathrm{x}$ & & & & & Locker and Martini (1986) \\
\hline
\end{tabular}

Table 2. Occurrence of Cenozoic silicoflagellates in Leg 113 cores.

\begin{tabular}{|c|c|c|c|c|c|c|}
\hline Hole & Latitude & Longitude & $\begin{array}{c}\text { Water } \\
\text { depth } \\
(\mathrm{m})\end{array}$ & $\begin{array}{c}\text { Number } \\
\text { of } \\
\text { cores }\end{array}$ & $\begin{array}{c}\text { Recovery } \\
(\%)\end{array}$ & $\begin{array}{l}\text { Silicoflagellate } \\
\text { occurrence }\end{array}$ \\
\hline $689 A$ & $64^{\circ} 31.01^{\prime} \mathrm{S}$ & $03^{\circ} 05.99^{\prime} \mathrm{E}$ & 2080 & 1 & 98.4 & Pleistocene-Pliocene \\
\hline $689 \mathrm{~B}$ & $64^{\circ} 31.01^{\prime} \mathrm{S}$ & $03^{\circ} 05.99^{\prime} \mathrm{E}$ & 2080 & 33 & 77.2 & Pleistocene-upper Eocene \\
\hline $689 \mathrm{C}$ & $64^{\circ} 31.01^{\prime} \mathrm{S}$ & $03^{\circ} 06.03^{\prime} \mathrm{E}$ & 2080 & 3 & 75.8 & Pleistocene-Miocene \\
\hline 689D & $64^{\circ} 31.01^{\prime} \mathrm{S}$ & $03^{\circ} 06.03^{\prime} \mathrm{E}$ & 2080 & 12 & 101.4 & Pliocene-upper Eocene \\
\hline $690 \mathrm{~A}$ & $65^{\circ} 09.63^{\prime} \mathrm{S}$ & $01^{\circ} 12.30^{\prime} \mathrm{E}$ & 2914 & 1 & 128.0 & Pleistocene-Pliocene \\
\hline $690 \mathrm{~B}$ & $65^{\circ} 09.63^{\prime} \mathrm{S}$ & $01^{\circ} 12.30^{\prime} \mathrm{E}$ & 2914 & 25 & 100.5 & Pleistocene-Oligocene \\
\hline $690 \mathrm{C}$ & $65^{\circ} 09.62^{\prime} \mathrm{S}$ & $01^{\circ} 12.29^{\prime} \mathrm{E}$ & 2914 & 24 & 88.3 & Pleistocene-Oligocene \\
\hline $693 \mathrm{~A}$ & $70^{\circ} 49.89^{\prime} \mathrm{S}$ & $14^{\circ} 34.41^{\prime} \mathrm{W}$ & 2359 & 51 & 44.1 & $\begin{array}{c}\text { Pleistocene-Oligocene; } \\
\text { Lower Cretaceous }\end{array}$ \\
\hline 693B & $70^{\circ} 49.89^{\prime} \mathrm{S}$ & $14^{\circ} 34.46^{\prime} \mathrm{W}$ & 2539 & 19 & 55.1 & $\begin{array}{l}\text { Miocene-Oligocene; } \\
\text { Lower Cretaceous }\end{array}$ \\
\hline $694 \mathrm{~A}$ & $66^{\circ} 50.83^{\prime} \mathrm{S}$ & $33^{\circ} 26.80^{\prime} \mathrm{W}$ & 4653 & 1 & 100.0 & Pleistocene-Pliocene \\
\hline 694B & $66^{\circ} 50.84^{\prime} \mathrm{S}$ & $33^{\circ} 26.83^{\prime} \mathrm{W}$ & 4653 & 25 & 34 & Pleistocene-Miocene \\
\hline $694 \mathrm{C}$ & $66^{\circ} 50.82^{\prime} \mathrm{S}$ & $33^{\circ} 26.76^{\prime} \mathrm{W}$ & 4653 & 23 & 33.7 & Miocene \\
\hline $695 \mathrm{~A}$ & $62^{\circ} 23.48^{\prime} \mathrm{S}$ & $43^{\circ} 27.10^{\prime} \mathrm{W}$ & 1305 & 42 & 73.7 & Pleistocene-Pliocene \\
\hline $696 \mathrm{~A}$ & $61^{\circ} 50.95^{\prime} \mathrm{S}$ & $42^{\circ} 55.98^{\prime} \mathrm{W}$ & 650 & 12 & 55.0 & Pleistocene-Pliocene \\
\hline $696 \mathrm{~B}$ & $61^{\circ} 50.96^{\prime} \mathrm{S}$ & $42^{\circ} 56.00^{\prime} \mathrm{W}$ & 650 & 62 & 27.5 & Pliocene-Miocene \\
\hline $697 \mathrm{~A}$ & $61^{\circ} 48.63^{\prime} \mathrm{S}$ & $40^{\circ} 17.73^{\prime} \mathrm{W}$ & 3481 & 3 & 94.7 & Pleistocene \\
\hline 697B & $61^{\circ} 48.63^{\prime} \mathrm{S}$ & $40^{\circ} 17.73^{\prime} \mathrm{W}$ & 3483 & 32 & 61.7 & Pleistocene-lower Pliocene \\
\hline
\end{tabular}

was added and the beaker heated for $30 \mathrm{~min}$. Samples were then centrifuged and decanted twice and washed once before making strewn slides. For similar methods of slide preparation, see Bukry (1983) and Busen and Wise (1977). For Sites 693 to 697, in which there was little calcareous material, strewn slides were made directly from the raw sediment.
A major goal of this study has been to provide quantitative information on the assemblages. Although a general practice is to count 300 specimens per slide, the silicoflagellate abundance in this study area was often not sufficient to allow such counts without a prohibitive investment of time. Except where the silicoflagellates were extremely abundant, all specimens on a slide 
(under a $22 \times 40 \mathrm{~mm}$ coverslip) were counted. Where the silicoflagellates were abundant, a second and even third slide might be counted. Fragments representing more than half of the original skeleton were included in the counts.

The desire to quantify the assemblage data necessarily meant that less emphasis could be placed on tracing out rare zonal markers, which was the goal during the shipboard studies. More detailed applications of zonal schemes applied to core catcher samples have been published in Barker, Kennett, et al. (1988). These qualitative biostratigraphic data from the shipboard work are included in the text of the present paper but are not always indicated on the range charts. Ages given on the range charts reflect the consensus ages provided by all of the microfossil groups plus the paleomagnetic data and are not derived solely from the silicoflagellate biostratigraphy. Where conflicts exist, the ages provided by the radiolarian stratigraphy are given (Lazarus, this volume).

While a number of high-latitude silicoflagellate zonations are available in the literature (Bukry, 1975c; Ciesielski, 1975; Martini and Muller, 1976), the one by Bukry combined with a modification of the Pliocene scheme of Ciesielski was the most applicable to our quantitative study (Table 3). Alternate zonations that could be applied for a given section are discussed in the text. The silicoflagellate biostratigraphy of Leg 113 is shown in Table 4 and discussed in more detail below.

\section{SITE SUMMARIES}

The locations and water depths of holes considered in this study are given in Table 2. Some of these holes were not examined in detail for silicoflagellates either because the core recovery was very low, they overlapped other holes at the same site, or the silicoflagellates were very rare.

\section{Site 689 (Table 5)}

Both the diversity and the abundance of silicoflagellates are higher at Hole 689B than elsewhere for ODP Leg 113. Silicoflagellates are well represented in the Pliocene and lower Oligo-

Table 3. Silicoflagellate zonation used for Leg 113.

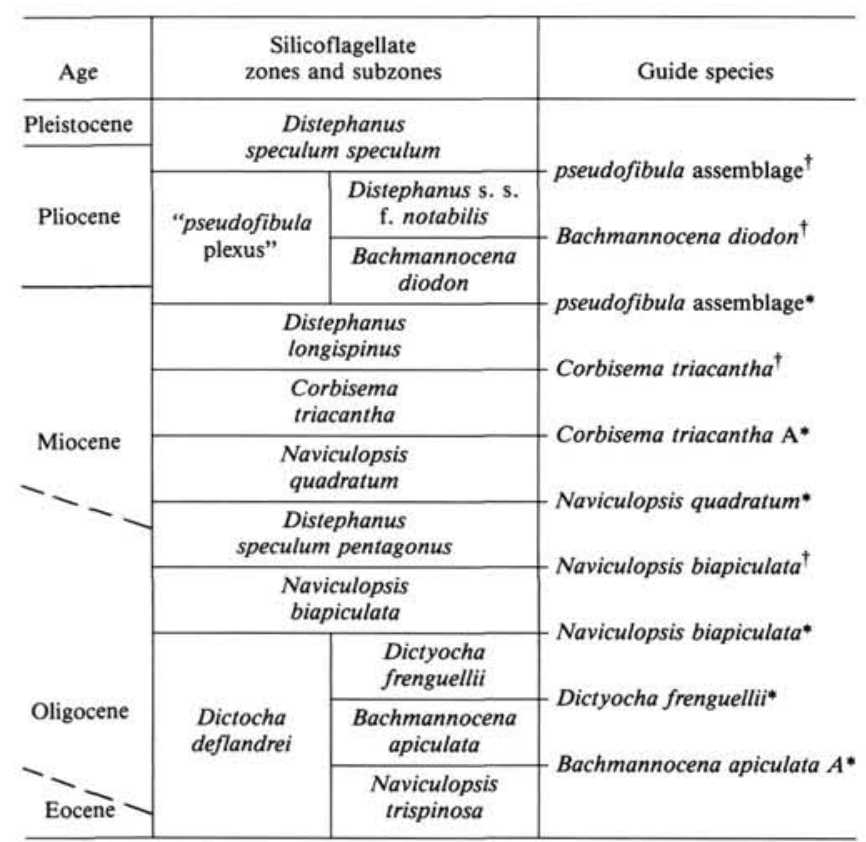

* = First appearance.

$\dagger=$ Last appearance. cene at Hole 689B, but are less common through most of the Miocene except at selected intervals. These can be zoned using the zonation of Bukry (1975c).

Cores from Section 113-689B-1H-1 to Section 113-689B-2H-5 are dated by radiolarian biostratigraphy as lower Pliocene in age (Shipboard Scientific Party, 1988a), and they contain large numbers of specimens that sometimes comprise veritable silicoflagellate oozes. These are strongly dominated by Distephanus speculum speculum down to Section 113-689B-2H-2 and by D. boliviensis below that. Sections 113-689B-2H-6 to 113-689B-3H-5 are dominated by forms of $D$. speculum speculum that are referred to here as the "pseudofibula plexus." These cores belong to the Bachmannocena diodon Subzone. Small numbers of the pseudofibula plexus occur in Sections 113-689B-2H-4 and 113$689 \mathrm{~B}-2 \mathrm{H}-5$. We assume that these are reworked above a disconformity between Sections 113-689B-2H-5 and 113-689B-2H-6 indicated by the diatom stratigraphy (Gersonde and Burckle, this volume). The relatively high abundance of Distephanus boliviensis just above the pseudofibula plexus found at Hole 689B is similar to that found by Ciesielski $(1974,1975)$.

Hole 689B was unusual among those drilled during Leg 113 in that both members of the pseudofibula plexus and the Bachmannocena species $B$. diodon co-occur in large numbers. Large Bachmannocena specimens are rare or absent at the other Leg 113 sites. These have been used as zonal indicators (Ciesielski, 1975; Martini and Muller, 1976; Busen and Wise, 1977), but $B$. diodon is used here only as a subzonal marker because the occurrences of this and the other Miocene/Pliocene Bachmannocena species are generally sporadic, in part due to hiatuses. Neither $B$. circulus nor $B$. diodon occurs in many samples of Hole $689 \mathrm{~B}$, but where present they are abundant. According to the zonation of Ciesielski (1975), the high concentration of $B$. circulus in Sample 113-689B-6H-2, 29-31 cm, would indicate his upper Miocene $B$. circulus Zone. This core, however, is dated as middle Miocene by combined radiolarian, diatom, and magnetostratigraphic analysis (Shipboard Scientific Party, 1988a, p. 126, 127 , and 143). This indicates that abundant $B$. circulus appears in the Southern Ocean earlier than previously documented, which is more in accordance with its range recorded elsewhere in the world (e.g., McCartney and Wise, 1987).

A hiatus at approximately 21 mbsf (Core 113-689B-3H), which separates lower Pliocene from upper Pliocene sediments, has been inferred from radiolarian and paleomagnetic data (Shipboard Scientific Party, 1988a, p. 123; Gersonde et al., this volume). This hiatus seems to be corroborated by the silicoflagellate stratigraphy in that $B$. diodon is absent below $21 \mathrm{~m}$. Such a hiatus would also explain the absence of the $B$. diodon/B. circulus Zone of Busen and Wise (1977) at this site.

The Miocene is not zoned here in detail, although it may be possible to recognize the extinction of Corbisema triacantha in Section 113-689B-7H-5 based on a very small number of specimens. There is an acme of multiwindowed Distephanus speculum hemisphaericus in Sections 113-689B-7H-2 to 113-689B$8 \mathrm{H}-5$, but the authors believe this to be due to environmental reasons. Thus we do not use this abundance to describe a new biostratigraphic zone, although this is done elsewhere (see Ciesielski et al., 1989). The multiwindowed forms are not as abundant at other Leg 113 sites.

There appears to be a hiatus between Sections 113-689B-8H-2 and $113-689 \mathrm{~B}-8 \mathrm{H}-5$ where the uppermost Oligocene sediments have been removed, as Naviculopsis biapiculata was not found (see also Shipboard Scientific Party, 1988a, p. 123). Sections $113-689 \mathrm{~B}-8 \mathrm{H}-5$ to $113-689 \mathrm{~B}-14 \mathrm{H}-5$ are characterized by Bachmannocena apiculata, Distephanus crux, and Dictyocha frenguellii in the upper part. Much of the interval can be assigned to the Dictyocha deflandrei Zone of Bukry (1975c). The Dictyocha frenguellii Subzone extends downhole to Section 113-689B-12H-2, 
Table 4. Silicoflagellate zones found in sections shown from Leg 113.

\begin{tabular}{|c|c|c|c|c|c|c|c|c|c|}
\hline \multicolumn{2}{|c|}{$\begin{array}{l}\text { Silicoflagellate } \\
\text { zone or subzone }\end{array}$} & $\begin{array}{l}\text { Hole } \\
689 \mathrm{~B}\end{array}$ & $\begin{array}{l}\text { Hole } \\
690 \mathrm{~B}\end{array}$ & $\begin{array}{l}\text { Hole } \\
693 \mathrm{~A}\end{array}$ & $\begin{array}{l}\text { Hole } \\
694 \mathrm{~B}\end{array}$ & $\begin{array}{l}\text { Hole } \\
695 \mathrm{~A}\end{array}$ & $\begin{array}{l}\text { Hole } \\
696 \mathrm{~A}\end{array}$ & $\begin{array}{l}\text { Hole } \\
696 \mathrm{~B}\end{array}$ & $\begin{array}{l}\text { Hole } \\
697 \mathrm{~B}\end{array}$ \\
\hline \multicolumn{2}{|c|}{$\begin{array}{c}\text { Distephanus } \\
\text { speculum speculum }\end{array}$} & $\begin{array}{c}1 \mathrm{H}-1 \text { to } \\
2 \mathrm{H}-5\end{array}$ & $\begin{array}{c}2 \mathrm{H}-1 \text { to } \\
3 \mathrm{H}-3\end{array}$ & $\begin{array}{c}6 \mathrm{R}-2 \text { to } \\
12 \mathrm{R}-7\end{array}$ & & $\begin{array}{c}3 \mathrm{H}-2 \text { to } \\
30 \mathrm{X}-2\end{array}$ & $\begin{array}{c}1 X-2 \text { to } \\
9 \mathrm{X}-2\end{array}$ & $\begin{array}{c}2 \mathrm{R}-1 \text { to } \\
3 \mathrm{R}-5\end{array}$ & $\begin{array}{c}11 \mathrm{H}-3 \text { to } \\
32 \mathrm{X}-3\end{array}$ \\
\hline \multirow{2}{*}{$\begin{array}{l}\text { “pseudofibula } \\
\text { plexus" }\end{array}$} & $\begin{array}{l}\text { Distephanus s. s. } \\
\text { f. notabilis }\end{array}$ & & $\begin{array}{l}3 \mathrm{H}-4 \text { to } \\
3 \mathrm{H}-5\end{array}$ & \multirow{2}{*}{$\begin{array}{c}13 \mathrm{R}-1 \text { to } \\
18 \mathrm{R}-2\end{array}$} & \multirow{2}{*}{$14 \mathrm{H}-1$} & \multirow{2}{*}{$\begin{array}{l}32 X-2 \text { to } \\
35 X-2\end{array}$} & & \multirow{2}{*}{$\begin{array}{l}\text { SR-2 to } \\
10 \mathrm{R}-1\end{array}$} & \multirow{2}{*}{$32 X-5$} \\
\hline & $\begin{array}{l}\text { Bachmannocena } \\
\text { diodon }\end{array}$ & $\begin{array}{c}2 \mathrm{H}-6 \text { to } \\
3 \mathrm{H}-5\end{array}$ & & & & & & & \\
\hline \multicolumn{2}{|c|}{$\begin{array}{l}\text { Distephanus } \\
\text { longispinus }\end{array}$} & $\begin{array}{l}3 \mathrm{H}-6 \text { to } \\
7 \mathrm{H}-2\end{array}$ & $\begin{array}{c}3 \mathrm{H}-6 \text { to } \\
6 \mathrm{H}-2\end{array}$ & & & & & & \\
\hline \multicolumn{2}{|c|}{$\begin{array}{l}\text { Corbisema } \\
\text { triacantha }\end{array}$} & & $6 \mathrm{H}-5$ & & & & & & \\
\hline \multicolumn{2}{|c|}{$\begin{array}{l}\text { Naviculopsis } \\
\text { quadratum }\end{array}$} & & & & & & & & \\
\hline \multicolumn{2}{|c|}{$\begin{array}{c}\text { Distephanus } \\
\text { speculum pentagonus }\end{array}$} & & & & & & & & \\
\hline \multicolumn{2}{|c|}{$\begin{array}{c}\text { Naviculopsis } \\
\text { biapiculata }\end{array}$} & & & & & & & & \\
\hline \multirow{3}{*}{$\begin{array}{l}\text { Dictyocha } \\
\text { deflandrei }\end{array}$} & $\begin{array}{l}\text { Dictyocha } \\
\text { frenguellii }\end{array}$ & $\begin{array}{c}8 \mathrm{H}-5 \text { to } \\
12 \mathrm{H}-2\end{array}$ & $\begin{array}{l}7 \mathrm{H}-2 \text { to } \\
10 \mathrm{H}-2\end{array}$ & $\begin{array}{c}38 \mathrm{R}-1 \text { to } \\
40-1\end{array}$ & & & & & \\
\hline & $\begin{array}{c}\text { Bachmannocena } \\
\text { apiculata }\end{array}$ & \multirow{2}{*}{$\begin{array}{l}12 \mathrm{H}-5 \text { to } \\
15 \mathrm{H}-2\end{array}$} & & & & & & & \\
\hline & $\begin{array}{l}\text { Naviculopsis } \\
\text { trispinosa }\end{array}$ & & & & & & & & \\
\hline
\end{tabular}

based on the first occurrence of the nominative species. The boundary between the lower two subzones of the $D$. deflandrei Zone, however, cannot be drawn because Bachmannocena apiculata continues downhole to the disconformity at the base of the Oligocene section. There is a sharp abundance peak (acme) of Naviculopsis trispinosa in Section 113-689B-13H-2, however, which may coincide with the extinction of this taxon. Slightly lower in the section in Hole 689D, Core 113-689D-10H yielded a diverse assemblage, which includes Distephanus crux, Bachmannocena apiculata, and $B$. occidentalis. The upper Eocene Section 113-689D-12H, CC, contains well preserved Dictyocha quadria (not shown on range chart).

Ebridians are unusually abundant in Sections 113-689B-14H-5 to 113-689B-15-2. A very unusual assemblage of large ebridians was found in Sample 113-689B-14H-5, 30-32 cm. A similar assemblage has been described previously by Gombos (1982) from the middle Eocene of the Falkland Plateau. The co-occurring silicoflagellates, however, indicate that these samples are still part of the Dictyocha deflandrei Zone and thus are late Eocene or Oligocene in age. The ebridians Adonnadonna and Triskelion ( $\mathrm{Pl} .7)$ appear to range above the middle Eocene, assuming that the specimens are not reworked.

\section{Site 690 (Table 6)}

The silicoflagellate stratigraphy at this site is similar to that at the shallower Site 689 , except that abundances are lower in the Miocene and Oligocene. Silicoflagellates are well represented in the Pliocene at Site 690, where silicoflagellate-diatom oozes are dominated by Distephanus speculum from Sections 113690B-2H-1 to 113-690B-3H-2. The last appearance datum of Distephanus boliviensis is in Section 113-690B-2H-7, below which this taxon alternates in dominance with $D$. speculum downsection to Section 113-690B-3H-4 and 113-690B-3H-5. As at Site 689 , a wide diversity of six-sided morphologies without apical rings (the pseudofibula plexus) is very abundant in a relatively short interval from Section 113-690B-3H-4 to $-3 \mathrm{H}-5$. Unlike the situation at Site 689, however, Bachmannocena diodon is not present in this interval due to a disconformity within Section
113-690B-3H-5, whereby the lowermost Pliocene is missing (including the radiolarian lower Tau Zone (Lazarus, this volume)). This disconformity between 18.0 and $19.5 \mathrm{mbsf}$ is recorded in all siliceous microfossil groups (Gersonde et al., this volume). The interval containing the pseudofibula plexus, therefore, is assigned to the Distephanus s. speculum f. notabilis Subzone. This subzone appears to be bounded above as well as below by disconformities, hence the abrupt changes in the abundances of the pseudofibula plexus. There might be a slight discrepancy between the diatom and silicoflagellate stratigraphies, however, in the placement of the disconformity shown in Table 6 between 15.0 and $16.5 \mathrm{mbsf}$ (see Gersonde et al., this volume).

At this site, the Distephanus longispinus Zone includes an exceptional abundance of five-sided Distephanus speculum. The Miocene Corbisema triacantha Zone may be represented by Sample 113-690B-6H-5, 28-30 cm. Much of the Miocene and Oligocene, however, appears to be absent due to hiatuses (see Tables 4, 6). Among the lower Miocene and upper Oligocene zones that are missing are the Naviculopsis biapiculata Zone and the $N$. trispinosa Subzone of the Dictyocha deflandrei Zone. Bachmannocena apiculata and Dictyocha deflandrei cooccur in Section 113-690A-9H-02, indicating an early Oligocene age, but the silicoflagellate abundances are very low. The Naviculopsis trispinosis acme noted at Site 689 is missing due to the hiatus between the Miocene and Oligocene.

\section{Site 693 (Table 7)}

The silicoflagellate biostratigraphy at Site 693 on the Antarctic margin is very similar to that found at Site 690 . The Pleistocene section is barren of silicoflagellates, whereas Distephanus speculum speculum is present in Sections 113-693A-6R-2 to 113693A-12R-2, but the abundances are much less than that in comparable cores from Holes 689B and 690A. Distephanus boliviensis is abundant in Section 113-693A-12R-7, which is immediately above the pseudofibula plexus. This abundance of $D$. boliviensis above the pseudofibula plexus is also found in Hole 689B and in DSDP Leg 28, and this interval could be assigned to the D. boliviensis Zone of Ciesielski (1975). The pseudofi- 
bula plexus occurs over a relatively large interval, spanning Sections 113-693A-13R-1 to 113-693A-15R-2 and perhaps extending to $113-693 \mathrm{~A}-18 \mathrm{R}-2$.

Although not shown in detail on the range chart, a number of important datums marked by rare to few Bachmannocena were noted in core-catcher samples. The last appearance datum (LAD) of $B$. diodon indicated by rare specimens in Section 113693A-17R, CC, marks the lower Pliocene top of the $B$. diodon Zone of Ciesielski (1975) (or of the B. diodon Subzone of this study), which straddles the Pliocene/Miocene boundary. The top of the subjacent $B$. circulus-B. diodon Zone of Busen and Wise (1977) can probably be placed at Section 113-693A-22R, CC (uppermost Miocene), which contains rare B. circulus var. $d u$ mitrica. The Pliocene/Miocene boundary on the range chart is that given by the Shipboard Scientific Party (1988b). Silicoflagellates are infrequent throughout the remainder of the Miocene section, and it is not possible to zone this interval.

No range chart was prepared for Hole 693B, but the following observations were made on core-catcher samples from that hole. The lowermost(?) Miocene Section 113-693B-9X, CC, contains one nearly complete specimen of Corbisema inermis crenulata, which has only been reported from the Paleocene and is presumed to have been reworked into this section from sediments higher upslope on the margin of Antarctica.

Core 113-693B-11X (316.1-325.8 mbsf) contains Bachmannocena apiculata apiculata and Distephanus crux darwinii. On the Falkland Plateau, the latter taxon has been reported only from the upper Oligocene (Busen and Wise, 1977). Upper Oligocene Cores 113-693B-12X and 113-693B-13X contain D. crux and Naviculopsis biapiculata.

In the Oligocene of Hole 693A, the occurrence of Bachmannocena apiculata, Dictyocha deflandrei, and Dictyocha frenguellii in Sections 113-693A-38H-1 to $113-693 \mathrm{~A}-40 \mathrm{H}-1$ indicates that this interval belongs to the Dictyocha frenguellii Subzone of the Dictyocha deflandrei Zone, which is near the lower Oligocene/upper Oligocene boundary. Silicoflagellates are well preserved, and ebridians are also found in this interval. The lower Oligocene Naviculopsis trispinosa acme observed at Site 689 was not noted at Site 693. This indicates that, due to a hiatus, the lowermost Oligocene was not cored at this site. The ebridian Ammodochium ampulla is common at the base of the sequence.

The Cretaceous diatomaceous sediments from Lithostratigraphic Unit 6 contain no members of the diverse silicoflagellate assemblage described from the Maestrichtian siliceous ooze at DSDP Site 275 on the Campbell Plateau (Hajos, 1975). Conspicuously absent at Site 693 are Upper Cretaceous taxa reported from Site 275 and elsewhere such as Corbisema geometrica, and species of Lyramula. This negative evidence indicates that the diatomaceous sediments recovered at Site 693 are older than Santonian or Campanian in age. According to the coccolith and palynomorph dates on the underlying "black shales," however, they can be no older than late Aptian in age (Mutterlose and Wise, this volume; Mohr, this volume, chapter 29), and Mohr (this volume, chapter 29) suggests an early Albian age for the deposit. These richly diatomaceous sediments do contain a rich assemblage of what appears to be an ancestral silicoflagellate stock, which McCartney et al. (this volume, chapter 27) discuss in some detail.

\section{Site 694 (no range chart)}

Silicoflagellates were largely absent at this deep water site, and no zonation is attempted. Members of the pseudofibula plexus are present, however, in a very narrow interval that includes Section 113-694B-14H-1, 67-69 cm. This is just below the thick sequence of sand turbidites of Lithostratigraphic Unit II. This occurrence supports an age near the Miocene/Pliocene boundary (Gensonde et al., this volume). The sands are interpreted as the products of high-energy turbidity currents (see Barker, Kennett, et al., 1988). It is interesting to note that at the three sites drilled to the north of Site 694 (see summaries for Sites 695-697 below), pseudofibula plexus is present in several cores immediately above a prominent interval of ice-rafted debris attributed to late Miocene-early Pliocene glaciation.

\section{Site 695 (Table 8)}

Silicoflagellates are generally present but are of low diversity in Sections 113-695A-1H to 113-695A-35X-2 (Lithostratigraphic Units I and II), and are absent in the clays and glacial marine sediments of Lithostratigraphic Unit III (Cores 113-695A-37X to $113-695 \mathrm{~A}-41 \mathrm{X})$. Overall, silicoflagellates are most common and well preserved where diatoms are well preserved and are absent or present mostly as fragments where diatom preservation is only moderate or poor. As a group, the silicoflagellates appear to be more sensitive to dissolution than the diatoms (Schrader, 1972).

The Pliocene interval of Site 695 is much longer than in the previous Leg 113 sites. Short-spined Distephanus speculum speculum dominate the assemblages from Cores 113-695A-3H to 113-695A-7H. This interval can be assigned to the $D$. speculum speculum Zone of Bukry (1975c) or, alternatively, to D. speculum B of Ciesielski (1975) and Ciesielski and Weaver (1983). Silicoflagellates are absent in Cores 113-695A-8H to 113-695A$12 \mathrm{H}$ where diatom preservation is poor. Distephanus speculum and $D$. boliviensis co-occur in Sections 113-695A-12H, CC (not on range chart) to 113-695A-20X-2, but $D$. boliviensis dominates from 113-695A-22X-2 to $113-695 \mathrm{~A}-29 \mathrm{X}-2$, although its abundance is low. This interval, where $D$. boliviensis is dominant, is zoned here as part of the Distephanus speculum speculum Zone (Bukry, 1975c) but can alternatively be assigned to the Distephanus boliviensis Zone of Ciesielski (1974, 1975). The zonation of Bukry is preferred by the authors because the two $\mathrm{Di}$ stephanus taxa sometimes appear to intergrade and because the pygmaea and pumila variants of Dictyocha (see Ciesielski, 1975; Ciesielski and Weaver, 1983) are absent throughout the Leg 113 samples.

Sections 113-695A-32X-4 to 113-695A-35X-2 are dominated by members of the pseudofibula plexus. Shaw and Ciesielski (1983) place the pseudofibula acme in the middle Gilbert. This corresponds well to the radiolarian assignment for these cores (upper portion of the lower Tau; see Lazarus, this volume). Core-catcher samples below Core 113-695A-35X were barren.

\section{Site 696 (Tables 9 and 10)}

Holes 696A and 696B present a biostratigraphy similar to that found in previous Leg 113 sites, but only down to the middle Miocene. Distephanus speculum dominates through Hole 696A except for the deepest sample studied. At Hole 696B, Distephanus boliviensis is dominant in Section 113-693B-3R-5. Aboard ship an attempt was made to apply the zonation of Ciesielski (1975) to the sequence with the following results (Shipboard Scientific Party, 1988c): Core 113-696A-1H contained Distephanus speculum speculum and $D$. septenarius, and is assigned to the D. speculum A Zone of Ciesielski (1975). Short-spined D. speculum dominate Cores 113-696A-2H to 113-696A-6H, and these are assigned to the $D$. speculum B Zone. The Distephanus boliviensis Zone extends from 55 to $101 \mathrm{mbsf}$, with an acme in the numbers of silicoflagellates in Cores 113-696A-11X and 113-696A-12X as well as in Core 113-696B-3R. This sequence could not be discerned at all sites, however, and is not indicated on the range charts. The pseudofibula plexus occurs from Sections 113-696B$5 R-2$ to $113-696 \mathrm{~B}-12 \mathrm{R}, \mathrm{CC}$ (not on range chart), which is a thick interval similar to that found at Hole 693A. The silicoflagellate- 
Table 5. Abundance of silicoflagellates and ebridians in selected samples from Hole 689B. Species (in alphabetical order) are recorded as total number of specimens found in slides examined.

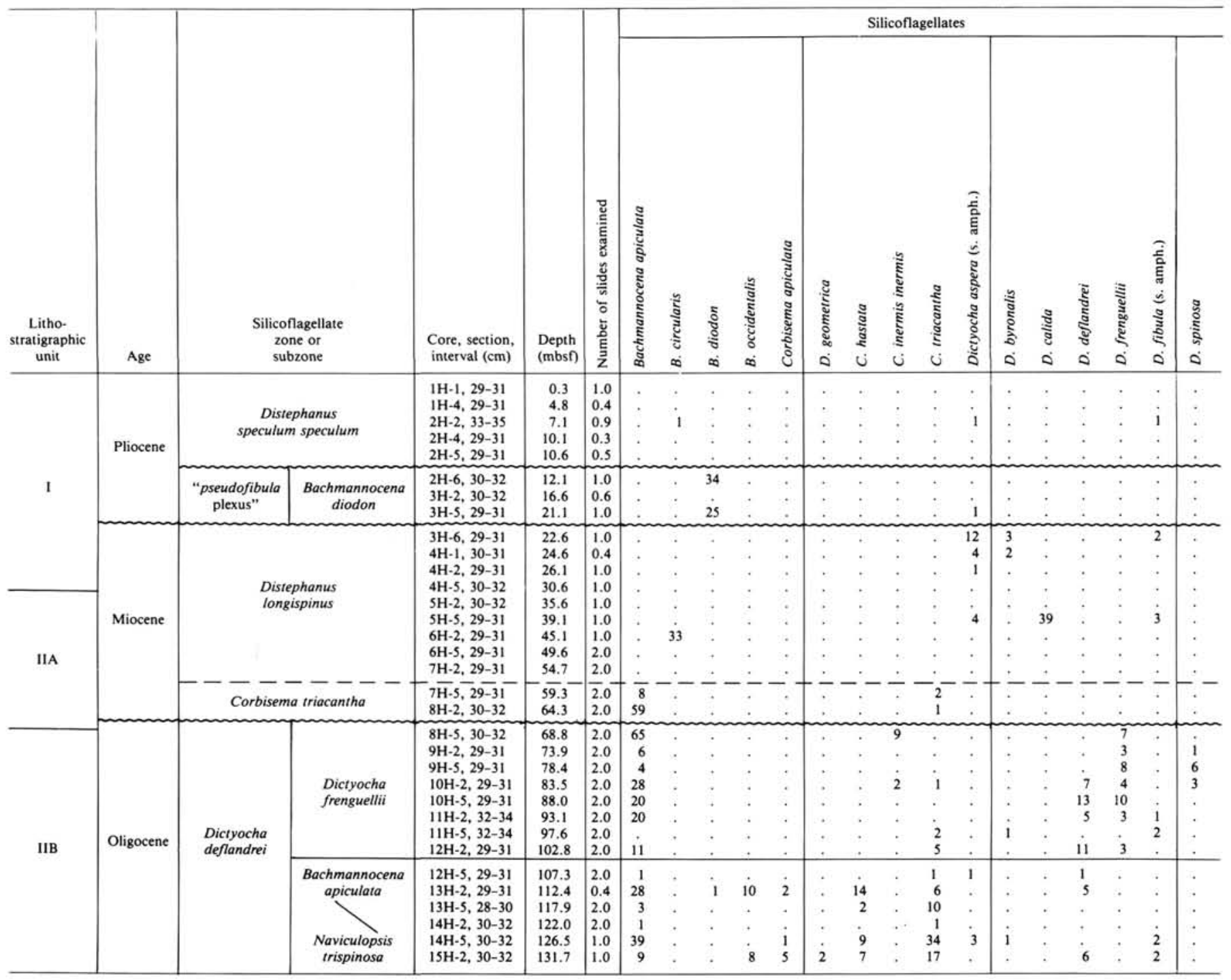

bearing samples below the pseudofibula plexus contained only five- and six-sided Distephanus and consequently could not be zoned.

\section{Site 697 (Table 11)}

Silicoflagellates are generally sparse or absent in the upper $250 \mathrm{~m}$ of the siliceous muds at Site 697; therefore, these fossils do not provide useful datums for biostratigraphy in Sections 113-697A-1H-1 to 113-697B-11H-3. This paucity of silicoflagellates is probably due to the relatively deep water of this site $(3480 \mathrm{~m})$, which would have promoted the dissolution of these fossils. Two diatomaceous intervals from Sections 113-697B$13 \mathrm{X}-1$ to $113-697 \mathrm{~B}-15 \mathrm{X}-3$ and 113-697B-21X-2 to 113-697B-22X-2, however, did contain silicoflagellates in moderate abundance. These intervals indicate enhanced productivity and preservation of biosiliceous material.

Six-sided Distephanus was relatively abundant from Sections 113-697B-13X-1 to 113-697B-22X-2. D. speculum predominates in the upper part of this interval whereas $D$. boliviensis is most abundant in the lower part; there is no distinct dividing point between these areas of dominance. Silicoflagellates are sparse or absent from Sections 113-697B-25X-1 to 113-697B-32X-3, except for 113-697B-28X-5 where $D$. boliviensis occurs in modest numbers.

Members of the pseudofibula plexus occur in low numbers in the deepest sample shown on the range chart (Table 11) as well as in Sections 113-697B-30X, CC and 113-697B-31X, CC (not on range chart); therefore, these last three cores can be assigned to this interval. The occurrence of the pseudofibula plexus coincides closely with the lower portion of the radiolarian Tau Zone (see Lazarus, this volume).

\section{SILICOFLAGELLATE SKELETAL VARIABILITY AND THE PSEUDOFIBULA PLEXUS}

\section{Explanatory Note}

One of the most unusual yet consistently present assemblages in the Leg 113 sequences is composed primarily of the group we refer to as the "pseudofibula plexus." This consists of the following five forms of Distephanus speculum speculum, which have been named formally:

D. s. s. forma pseudofibula

D. s. s. forma varians 


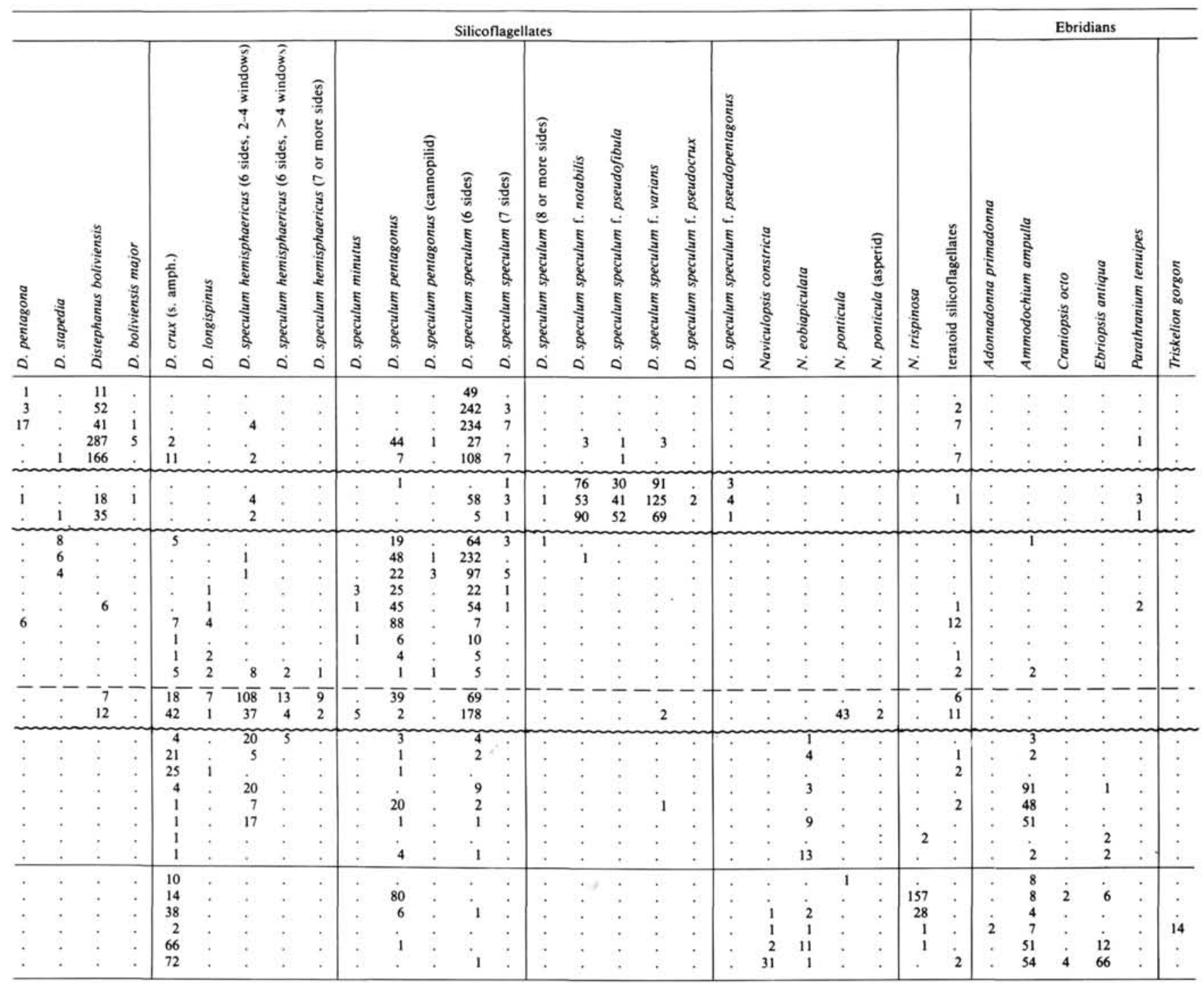

D. s. s. forma notabilis

D. s. s. forma pseudocrux

D. s. s. forma pseudopentagonus, n. f.

These seemingly distinct and different forms are unusual in that they belong to one subspecies. As such, they demonstrate the considerable morphologic plasticity that can occur within silicoflagellate taxa.

It is well known that silicoflagellates show exceptional variability in modern populations. However, in recent paleontologic literature and especially in silicoflagellate research concerning deep sea drilling, there has been an emphasis on subdividing taxa. Thus the number of silicoflagellate species in the paleontologic literature has increased enormously. This taxonomy may well bear little relation to actual biological relationships. In this section, by way of background information, we review the general silicoflagellate skeletal morphology and its variability. We then discuss the pseudofibula plexus found in Leg 113 and elsewhere, as it is an excellent example of silicoflagellate variability in the fossil record.

\section{Silicoflagellate Skeletal Morphology}

The silicoflagellate skeleton is composed of tubular silica elements that are rigidly interconnected to form a three-dimensional framework. This framework consists of a planar polygonal basal ring and an apical structure that forms a dome-like latticework above it. The apical structure usually consists of struts, which attach to the sides of the basal ring at some point between the corners, and an apical bridge or ring. The apical ring, if present, is usually a polygon similar to, and in a parallel plane with, the basal ring. In some forms, including most Corbisema, the struts meet at an apex without forming a bridge. Various workers (Mandra and Mandra, 1972; Poelchau, 1974; Bukry, 1976d; Perch-Nielsen, 1985; among others) have previously illustrated the descriptive terminology; the terminology used in this article is shown in Figure 3.

The term "element" is here used to denote a single skeletal rod that makes up a part of the silicoflagellate skeleton. Each skeletal element generally intersects other elements at each end. In all but a few cases, these intersections consist of three ele- 
Table 6. Abundance of silicoflagellates and ebridians in selected samples from Hole 690B. Species (in alphabetical order) are recorded as total number of specimens found in slides examined. * = Ebridian.

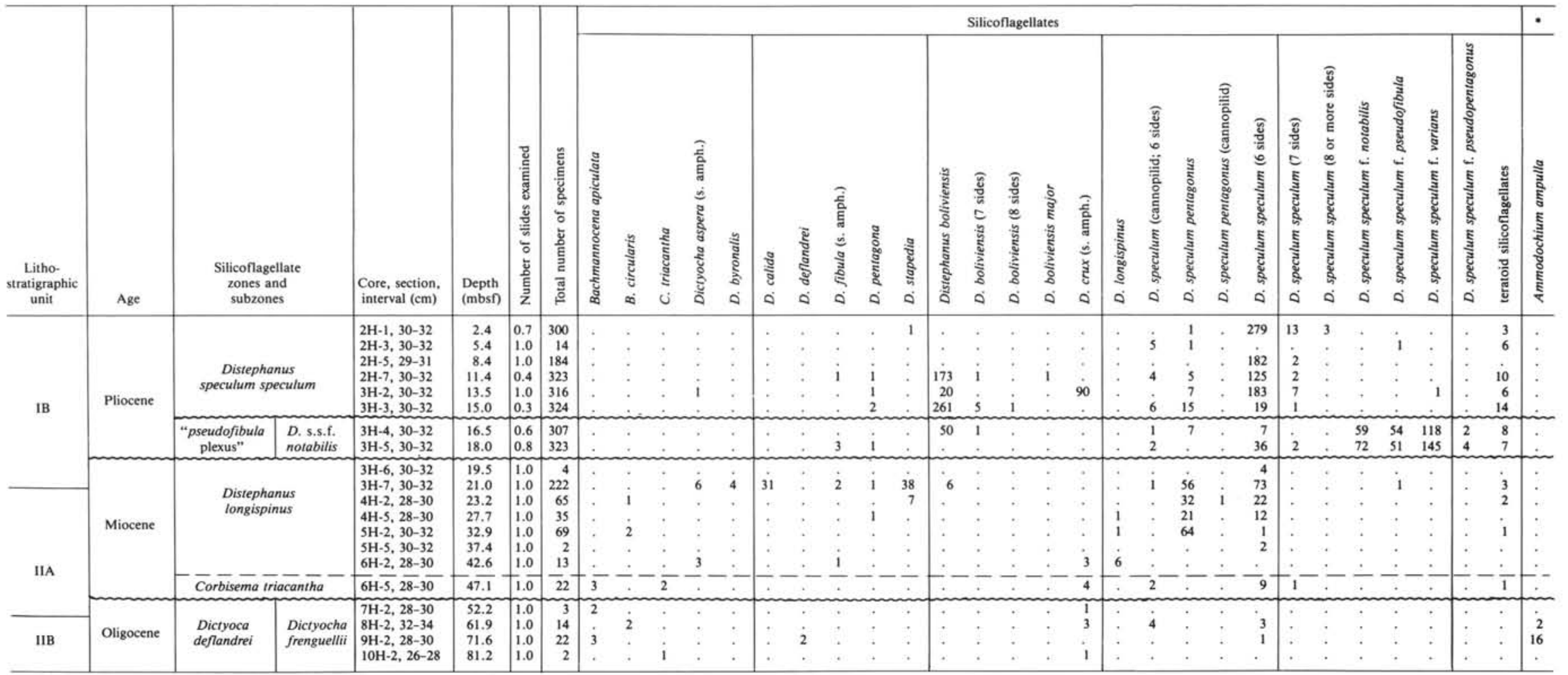


Table 7. Abundance of silicoflagellates in selected samples from Hole 693A. Species (in alphabetical order) are recorded as total number of specimens found in slides examined. ${ }^{\star}=$ Ebridian.

\begin{tabular}{|c|c|c|c|c|c|c|c|c|c|c|c|c|c|c|c|c|c|c|c|c|c|c|c|c|c|c|c|c|c|c|}
\hline \multirow[b]{2}{*}{$\begin{array}{l}\text { Litho- } \\
\text { stratigraphic } \\
\text { unit }\end{array}$} & \multirow[b]{2}{*}{ Age } & \multirow{2}{*}{\multicolumn{2}{|c|}{$\begin{array}{l}\text { Silicoflagellate } \\
\text { zones and } \\
\text { subzones }\end{array}$}} & \multirow[b]{2}{*}{$\begin{array}{l}\text { Core, section, } \\
\text { interval }(\mathrm{cm})\end{array}$} & \multirow[b]{2}{*}{$\begin{array}{l}\text { Depth } \\
\text { (mbsf) }\end{array}$} & \multirow[b]{2}{*}{ 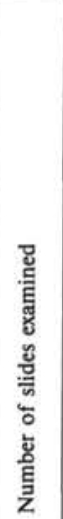 } & \multirow[b]{2}{*}{ 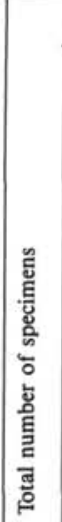 } & \multicolumn{22}{|c|}{ Silicoflagellates } & - \\
\hline & & & & & & & & 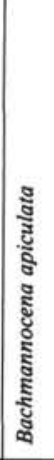 & $\begin{array}{l}\text { है } \\
\text { है } \\
\text { है } \\
\infty\end{array}$ & 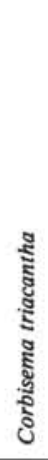 & 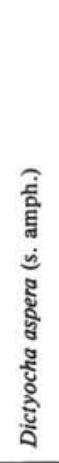 & 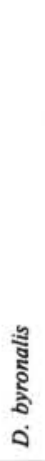 & 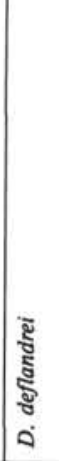 & 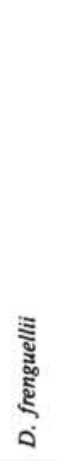 & 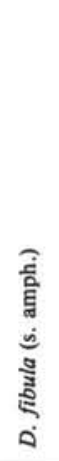 & 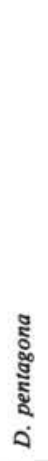 & 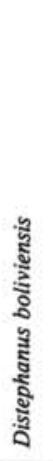 & 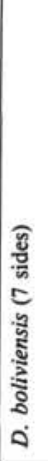 & 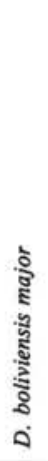 & 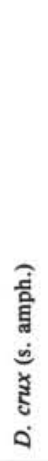 & 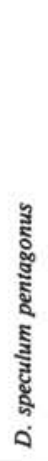 & 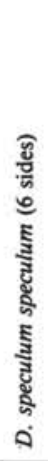 & 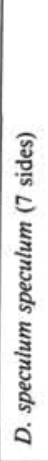 & 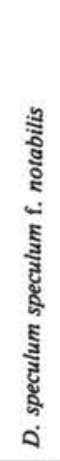 & 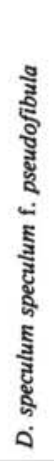 & 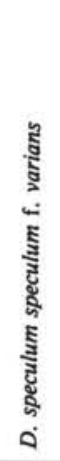 & 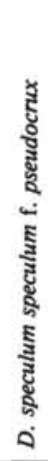 & 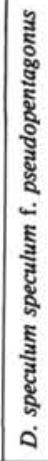 & 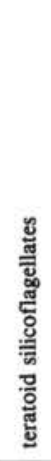 & 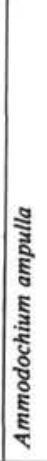 \\
\hline II & \multirow{4}{*}{ Pliocene } & \multirow{2}{*}{\multicolumn{2}{|c|}{$\begin{array}{c}\text { Distephanus } \\
\text { speculum speculum }\end{array}$}} & $\begin{array}{l}3 \mathrm{R}-2,124-126 \\
4 \mathrm{R}-2,30-32\end{array}$ & $\begin{array}{l}14.9 \\
23.7\end{array}$ & $\begin{array}{l}1.0 \\
1.0\end{array}$ & $\begin{array}{l}0 \\
0\end{array}$ & : & $\dot{.}$ & . & : & : & : & . & : & : & . & : & : & : & : & : & : & : & : & : & : & : & : & : \\
\hline \multirow{6}{*}{ IIIA } & & & & $\begin{array}{l}5 \mathrm{R}-2,30-32 \\
6 \mathrm{R}-2,30-32 \\
8 \mathrm{R}-2,30-32 \\
9 \mathrm{R}-2,30-32 \\
10 \mathrm{R}-2,30-32 \\
11 \mathrm{R}-3,30-32 \\
12 \mathrm{R}-2,28-30 \\
\end{array}$ & $\begin{array}{r}33.2 \\
43.9 \\
62.2 \\
71.9 \\
81.5 \\
92.6 \\
100.7 \\
\end{array}$ & $\begin{array}{l}1.0 \\
1.0 \\
1.0 \\
1.0 \\
1.0 \\
1.0 \\
1.0\end{array}$ & $\begin{array}{r}0 \\
6 \\
18 \\
64 \\
19 \\
13 \\
32 \\
\end{array}$ & $:$ & : & $:$ & $\begin{array}{l}: \\
\dot{4} \\
\dot{4} \\
\dot{ }\end{array}$ & $:$ & $:$ & : & $\begin{array}{l}: \\
: \\
8 \\
1\end{array}$ & $:$ & $\begin{array}{l}1 \\
17 \\
i \\
16\end{array}$ & $:$ & $:$ & $\begin{array}{r}\text { i } \\
\dot{7} \\
12\end{array}$ & $\begin{array}{l}\vdots \\
1 \\
1 \\
\vdots\end{array}$ & $\begin{array}{r}8 \\
6 \\
10 \\
43 \\
7 \\
4 \\
4\end{array}$ & : & $:$ : & $\begin{array}{l}i \\
i \\
i\end{array}$ & i & $:$ & $:$ : & $\begin{array}{l}1 \\
2 \\
2 \\
\vdots \\
:\end{array}$ & : \\
\hline & & \multicolumn{2}{|c|}{$\begin{array}{l}\text { "pseudofibula } \\
\text { plexus" }\end{array}$} & $\begin{array}{l}\text { 12R-7, } 30-32 \\
13 R-1,30-32 \\
13 R-2,30-32 \\
14 R-2,30-32 \\
15 R-2,30-32 \\
17 R-3,30-32 \\
18 R-2,30-32\end{array}$ & $\begin{array}{l}108.2 \\
108.7 \\
110.2 \\
120.0 \\
129.6 \\
150.5 \\
158.7\end{array}$ & \begin{tabular}{l|}
0.6 \\
1.0 \\
1.0 \\
1.0 \\
1.0 \\
1.0 \\
1.0
\end{tabular} & \begin{tabular}{|r|}
329 \\
310 \\
300 \\
37 \\
43 \\
0 \\
7 \\
\end{tabular} & : & : & : & : & : & $\vdots$ & $\dot{0}$ & : & $\begin{array}{l}\mathrm{i} \\
2 \\
\text { : } \\
\text { : }\end{array}$ & $\begin{array}{r}268 \\
74 \\
11 \\
6 \\
2 \\
. \\
\end{array}$ & $\begin{array}{l}7 \\
5 \\
5 \\
:\end{array}$ & $\begin{array}{l}3 \\
1 \\
\vdots \\
\vdots \\
\vdots\end{array}$ & : & $\begin{array}{l}11 \\
: \\
:\end{array}$ & $\begin{array}{r}29 \\
3 \\
2 \\
1 \\
1 \\
\end{array}$ & $\dot{ }$ & $\begin{array}{r}95 \\
111 \\
11 \\
18 \\
3 \\
3\end{array}$ & $\begin{array}{r}1 \\
38 \\
61 \\
8 \\
8 \\
. \\
\end{array}$ & $\begin{array}{r}88 \\
110 \\
12 \\
14 \\
4 \\
4\end{array}$ & $\begin{array}{l}\mathrm{i} \\
1 \\
\text {. } \\
\text {. }\end{array}$ & $\begin{array}{l}\mathrm{i} \\
2 \\
\text { : } \\
\vdots\end{array}$ & $\begin{array}{r}10 \\
8 \\
: \\
:\end{array}$ & : \\
\hline & & \multirow{12}{*}{$\begin{array}{l}\text { Dictyocha } \\
\text { deflandrei }\end{array}$} & \multirow{6}{*}{ oned } & 19R-2, 30-32 & 168.4 & 1.0 & 7 & . & . & . & . & . & . & . & . & . & 6 & . & . & . & . & 1 & . & . & & & . & . & . & . \\
\hline & \multirow{5}{*}{ Miocene } & & & $\begin{array}{l}21 \mathrm{R}-2,28-30 \\
22 \mathrm{R}-2,30-32\end{array}$ & $\begin{array}{l}187.7 \\
197.4\end{array}$ & $\begin{array}{l}1.0 \\
1.0\end{array}$ & $\begin{array}{l}0 \\
3\end{array}$ & : & . & . & : & · & . & · & . & . & i & . & · & i & . & . & . & . & . & . & : & . & . & . \\
\hline & & & & $25 \mathrm{R}-2,30-32$ & 226.4 & 1.0 & 0 & $\vdots$ & $\vdots$ & $\dot{.}$ & $\vdots$ & $\vdots$ & : & $\vdots$ & $\vdots$ & $\dot{.}$ & . & : & $\vdots$ & . & $\vdots$ & $\vdots$ & $\vdots$ & $\dot{0}$ & i. &. & $:$ & : & : & $\vdots$ \\
\hline & & & & $26 \mathrm{R}-1,30-32$ & 234.6 & 1.0 & 3 & . & . & . & . & . & . & . & 1 & . & 2 & . & . & . & . & . & . & . & . & . & . & . & . & . \\
\hline \multirow[t]{2}{*}{ IIIB } & & & & $\begin{array}{l}27 \mathrm{R}-2,30-32 \\
28 \mathrm{R}-2,30-32\end{array}$ & $\begin{array}{l}245.7 \\
255.4\end{array}$ & $\begin{array}{l}1.0 \\
1.0\end{array}$ & $\begin{array}{l}2 \\
0\end{array}$ & : & : & : & : & : & : & : & : & : & : & : & : & : & 2 & : & : & : & : & : & : & : & : & : \\
\hline & & & & $\begin{array}{r}29 \mathrm{R}-2,30-32 \\
31 \mathrm{R}-3,30-32\end{array}$ & $\begin{array}{l}264.7 \\
285.5\end{array}$ & $\begin{array}{l}1.0 \\
1.0\end{array}$ & $\begin{array}{r}65 \\
8\end{array}$ & $\begin{array}{r}46 \\
5\end{array}$ & $:$ & $:$ & : & . & : & : & . & . & 18 & . & $\therefore$ & 1 & i & . & . & . & . & $:$ & : & $\therefore$ & : & : \\
\hline \multirow[t]{3}{*}{ IIIC } & \multirow{6}{*}{ Oligocene } & & & $33 \mathrm{R}-1,96-98$ & 302.5 & 0.5 & 0 & ? & $\dot{\div}$ & $\because$ & $\div$ & . & $\div$ & $\because-$ & ? & . & & . & . & . & & & . & $\div$ & & $\div$ & 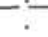 & $\div$ & & . \\
\hline & & & & $3,4 \mathrm{R}-2,118-120$ & 313.9 & 1.0 & 3 & 1 & . & . & . & . & . & . & . & . & 1 & . & . & . & . & 1 & . & . & . & . & . & . & . & . \\
\hline & & & & $\begin{array}{l}35 \mathrm{R}-2,30-32 \\
36 \mathrm{R}-1,30-32\end{array}$ & $\begin{array}{l}322.7 \\
330.8\end{array}$ & $\begin{array}{l}0.5 \\
0.5\end{array}$ & $\begin{array}{l}0 \\
0\end{array}$ & · & : & : & . & · & . & · & . & . & . & . & - & . & . & . & . & . & . & . & . & . & . & : \\
\hline IV & & & $\begin{array}{l}\text { Dictyocha } \\
\text { frenguelliii }\end{array}$ & $\begin{array}{l}36 \mathrm{R}-1,30-32 \\
37 \mathrm{R}-1,34-36\end{array}$ & $\begin{array}{l}334.8 \\
342.0\end{array}$ & $\begin{array}{l}0.5 \\
1.0\end{array}$ & $\begin{array}{l}0 \\
0\end{array}$ & $\dot{.}$ & : & * & : & : & : & : & : & : & $:$ & $\dot{5}$ & $\therefore$ & : & : & : & : & $\therefore$ & $:$ & : & : & : & : & $\dot{.}$ \\
\hline & & & & $38 \mathrm{R}-1,108-109$ & 351.0 & 1.0 & 58 & 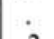 & . & 1 & . & 1 & 46 & 6 & . & i & . & . & . & 3 & . & . & . & . & . & . & . & . & : & 1 \\
\hline $\mathrm{v}$ & & & & $\begin{array}{l}39 \mathrm{R}-1,30-32 \\
40 \mathrm{R}-1,30-32\end{array}$ & $\begin{array}{l}359.9 \\
369.2\end{array}$ & 1.0 & $\begin{array}{l}23 \\
54\end{array}$ & 2 & $\cdot$ & . & · & . & 15 & i & . & 2 & . & . & . & 3 & . & . & . & $\cdot$ & . & . & . & . & . & $\begin{array}{r}1 \\
30\end{array}$ \\
\hline & & & & $40 \mathrm{k}-1,30-32$ & 309.2 & & 54 & & . & & . & & & & & & & $\cdot$ & $\cdot$ & & & & & & $\cdot$ & $\cdot$ & & $\cdot$ & & \\
\hline
\end{tabular}


Table 8. Abundance of silicoflagellates in selected samples from Hole 695A. Species (in alphabetical order) are recorded as total number of specimens found in slides examined.

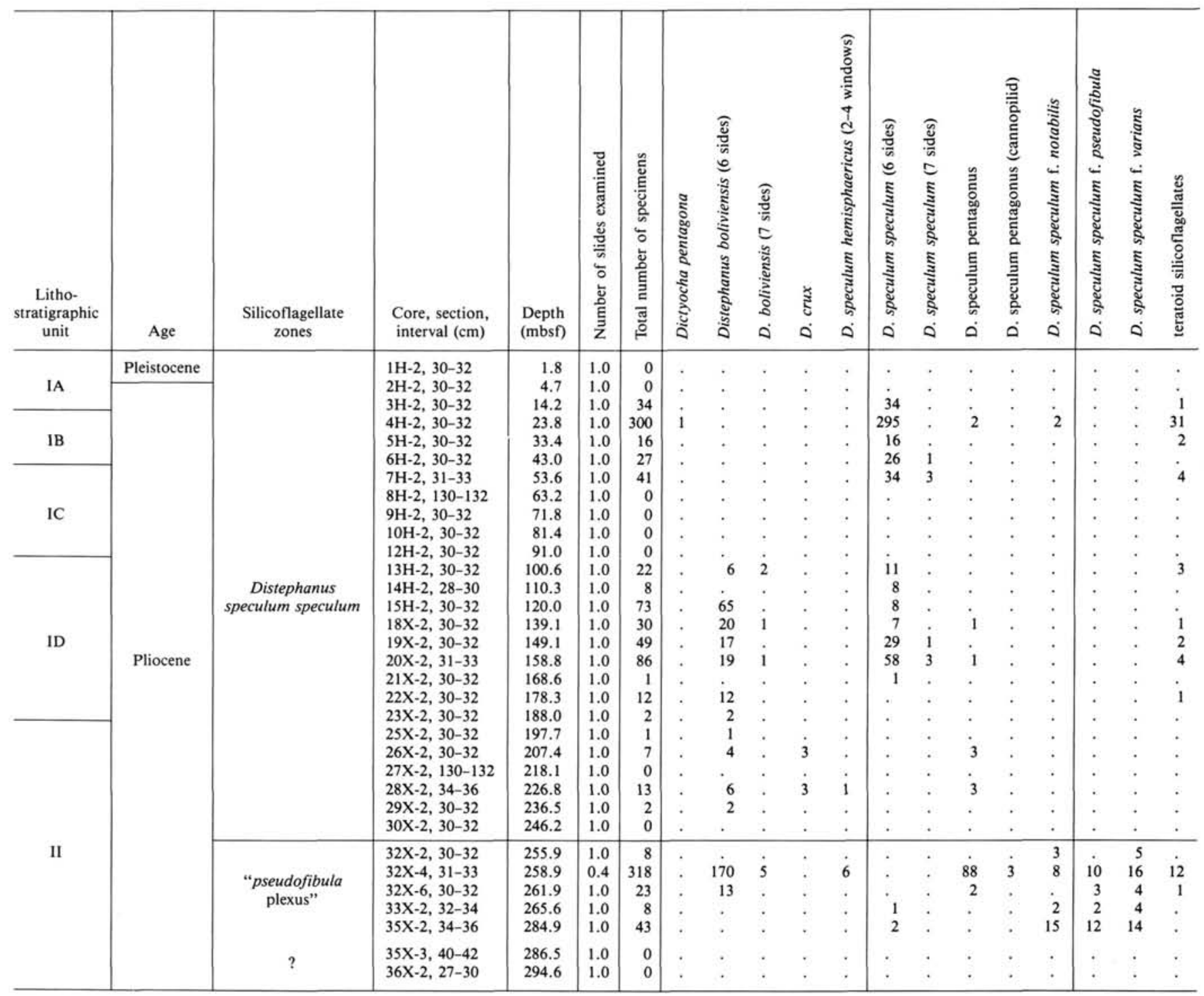

ments that meet to form a triple junction (see Poelchau, 1974); the angle between any two elements of this junction is seldom acute. Where two elements meet at an angle, there is usually a spine, which becomes the third member of the triple junction. This is commonly the case at the corners of the basal ring, or on the apical structure (such as on the apex of Dictyocha aculeata or Naviculopsis trispinosa). The silicoflagellate skeleton generally has triple junctions at the corners, at the points where the strut attaches to the basal side (here called "strut attachments"), and where the apical end of each strut attaches to the rest of the apical structure (bridge or ring attachment).

Although the skeletal morphology shows remarkable variability, the skeletal morphotypes appear to follow a few simple rules in their design. The number of struts, for example, is equal to the number of basal sides, and there is one strut per side. Similarly, the apical and basal rings have the same number of sides, and the entire skeleton in general has some form of rotational symmetry. In nearly all cases, the apex of the silicoflag- ellate, or the center of the apical ring, is directly above the center of the basal plane. While exceptions to these rules can be found, they are unusual and generally appear to be aberrant (teratoid) specimens.

The regularly occurring polygonal patterns found in silicoflagellate skeletal morphology are similar in many ways to configurations produced by mathematical optimization of one or more variables. Similar patterns occur among colonial corals or barnacles, the honeycomb structure of the beehive, or the venation of the insect wing (see Thompson, 1942; Smith, 1954; Raup, 1968; Almgren, 1982, for other examples). Some of these, such as the colonial corals, are the result of close packing. This, however, should not apply to silicoflagellates as they are not colonial and the polygons formed by the skeletal elements do not appear to reflect any segmentation within the organism. Instead, the silicoflagellate skeleton appears to be the result of area minimization, as has been previously proposed for some radiolarian skeletons (Thompson, 1942; Almgren, 1982), brach- 
Table 9. Abundance of silicoflagellates in selected samples from Hole 696A. Species (in alphabetical order) are recorded as total number of specimens found in slides examined.

\begin{tabular}{|c|c|c|c|c|c|c|c|c|c|c|c|c|c|c|}
\hline $\begin{array}{l}\text { Litho- } \\
\text { stratigraphic } \\
\text { unit }\end{array}$ & Age & $\begin{array}{l}\text { Silicoflagellate } \\
\text { zones }\end{array}$ & $\begin{array}{l}\text { Core, section, } \\
\text { interval }(\mathrm{cm})\end{array}$ & $\begin{array}{l}\text { Depth } \\
\text { (mbsf) }\end{array}$ & 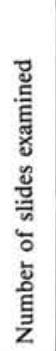 & 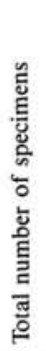 & 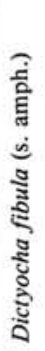 & 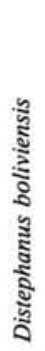 & 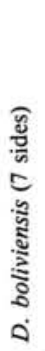 & 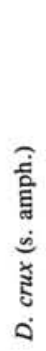 & 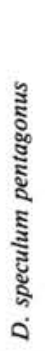 & 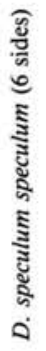 & 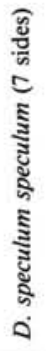 & 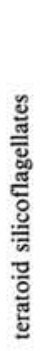 \\
\hline \multirow[t]{2}{*}{ IA } & \multirow[t]{2}{*}{ Quaternary } & \multirow{8}{*}{$\begin{array}{c}\text { Distephanus } \\
\text { speculum speculum }\end{array}$} & $1 \mathrm{H}-2,30-32$ & 01.8 & 1.0 & 6 & . & 4 & . & . & . & 2 & . & . \\
\hline & & & $2 \mathrm{H}-2,30-32$ & 4.3 & 1.0 & 0 & . & . & . & . & . & . & . & . \\
\hline \multirow{4}{*}{ IB } & \multirow{6}{*}{ Pliocene } & & $\begin{array}{l}3 \mathrm{H}-1,37-39 \\
4 \mathrm{H}-1,90-92\end{array}$ & $\begin{array}{l}12.4 \\
22.4\end{array}$ & $\begin{array}{l}1.0 \\
1.0\end{array}$ & $\begin{array}{r}6 \\
22\end{array}$ & : & : & : & : & : & $\begin{array}{r}6 \\
21\end{array}$ & i & : \\
\hline & & & $5 \mathrm{H}-2,30-32$ & 33.5 & 1.0 & 37 & . & 2 & $i$ & . & 1 & 31 & 1 & 1 \\
\hline & & & $6 \mathrm{H}-2,30-32$ & 42.6 & 1.0 & 16 & 1 & . & . & 2 & . & 11 & . & 2 \\
\hline & & & $7 \mathrm{H}-2,30-32$ & 52.2 & 1.0 & 5 & $\cdot$ & . & . & . & 1 & 3 & . & 1 \\
\hline \multirow{2}{*}{ II } & & & $8 \mathrm{H}-2,30-32$ & 61.8 & 1.0 & 20 & 1 & $\therefore$ & . & . & 9 & 9 & 1 & 1 \\
\hline & & & $9 \mathrm{H}-2,30-32$ & 71.4 & 1.0 & 23 & . & 12 & 1 & . & 10 & . & . & . \\
\hline
\end{tabular}

Table 10. Abundance of silicoflagellates in selected samples from Hole 696B. Species (in alphabetical order) are recorded as total number of specimens found in slides examined.

\begin{tabular}{|c|c|c|c|c|c|c|c|c|c|c|c|c|c|c|c|c|c|c|}
\hline $\begin{array}{l}\text { Litho- } \\
\text { stratigraphic } \\
\text { unit }\end{array}$ & Age & $\begin{array}{l}\text { Silicoflagellate } \\
\text { zones }\end{array}$ & $\begin{array}{l}\text { Core, section, } \\
\text { interval }(\mathrm{cm})\end{array}$ & $\begin{array}{l}\text { Depth } \\
\text { (mbsf) }\end{array}$ & 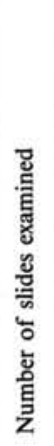 & 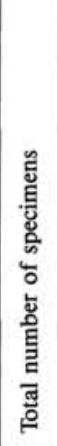 & 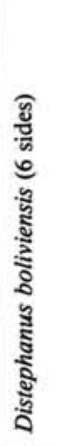 & 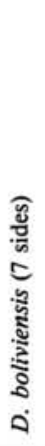 & 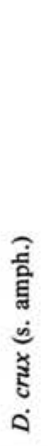 & 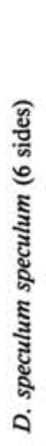 & 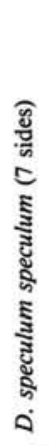 & 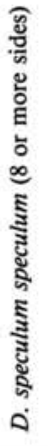 & 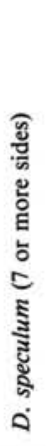 & 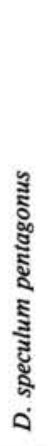 & 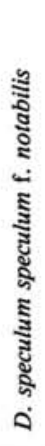 & 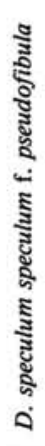 & 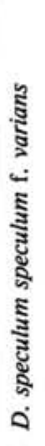 & 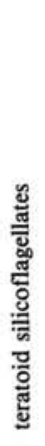 \\
\hline \multirow{3}{*}{ II } & Quaternary & \multirow{2}{*}{$\begin{array}{c}\text { Distephanus } \\
\text { speculum speculum }\end{array}$} & $\begin{array}{l}2 \mathrm{R}-1,74-76 \\
3 \mathrm{R}-2,129-131\end{array}$ & $\begin{array}{l}77.4 \\
89.0\end{array}$ & 1.0 & $\begin{array}{r}3 \\
18\end{array}$ & · & . & $\begin{array}{l}1 \\
2\end{array}$ & $\begin{array}{r}2 \\
15\end{array}$ & : & : & : & $i$ & : & : & . & : \\
\hline & \multirow{4}{*}{ Pliocene } & & $3 \mathrm{R}-5,129-131$ & 93.5 & 1.0 & 166 & 112 & 10 & . & 18 & 2 & . & 3 & 21 & . & . & . & . \\
\hline & & \multirow{3}{*}{$\begin{array}{l}\text { "pseudofibula } \\
\text { plexus" }\end{array}$} & $5 \mathrm{R}-2,30-32$ & 106.3 & 1.0 & 24 & . & . & - & . & . & . & . & . & 6 & 7 & 11 & . \\
\hline \multirow{3}{*}{ III } & & & 7R-2, 34-36 & 126.7 & $\begin{array}{l}1.0 \\
1.0\end{array}$ & 6 & : & $\dot{.}$ & $\dot{.}$ & : & $\dot{.}$ & $\dot{.}$ & $\dot{.}$ & : & $\begin{array}{l}2 \\
1\end{array}$ & $\begin{array}{l}3 \\
1\end{array}$ & 3 & 1 \\
\hline & & & $10 \mathrm{R}-1,31-33$ & 144.4 & 1.0 & 3 & . & . & . & . & . & . & . & . & 1 & . & 2 & 1 \\
\hline & \multirow{11}{*}{ Miocene } & \multirow{11}{*}{ Unzoned } & $13 R-1,42-44$ & 173.6 & 1.0 & 1 & . & . & . & . & 1 & . & . & . & . & . & . & . \\
\hline \multirow{3}{*}{ IV } & & & $17 \mathrm{R}-1,130-132$ & 213.1 & 1.0 & 15 & . & . & . & 14 & . & 1 & . & . & . & . & . & . \\
\hline & & & $19 \mathrm{R}-1,16-18$ & 221.7 & 1.0 & 19 & . & . & . & 18 & . & 1 & . & . & . & . & . & . \\
\hline & & & $20 \mathrm{R}-1,130-132$ & 232.4 & 1.0 & 9 & . & . & . & 6 & . & . & . & 3 & . & . & . & . \\
\hline \multirow{7}{*}{ VI } & & & $24 \mathrm{R}-2,30-32$ & 271.5 & 1.0 & 0 & . & . & . & . & . & . & . & . & . & . & . & . \\
\hline & & & $25 \mathrm{R}-1,30-32$ & 279.7 & 1.0 & 0 & . & . & . & . & . & . & . & . & . & . & . & . \\
\hline & & & $26 \mathrm{R}-2,30-32$ & 290.8 & 1.0 & 0 & . & . & . & & . & . & . & . & . & . & . & . \\
\hline & & & $27 \mathrm{R}-2,30-32$ & 300.5 & 1.0 & 31 & 25 & . & . & 6 & . & . & . & . & . & . & . & . \\
\hline & & & $28 \mathrm{R}-2,30-32$ & 310.1 & 1.0 & 1 & . & . & . & 1 & . & . & . & . & . & . & . & . \\
\hline & & & $32 \mathrm{R}-2,30-32$ & 348.8 & 1.0 & 5 & 5 & . & . & . & . & . & . & . & . & . & . & . \\
\hline & & & $34 \mathrm{R}-2,30-32$ & 368.1 & 1.0 & 2 & 2 & . & . & . & . & . & . & . & . & . & . & . \\
\hline
\end{tabular}

iopods (McGhee, 1980), foraminifers (Brasier, 1982), and is well known in soap bubbles (Almgren and Taylor, 1976; Isenberg, 1976; Fig. 4).

Recent work (McCartney and Loper, 1989) has shown that silicoflagellate skeletal configurations can be produced by a simple mathematical model that minimizes the apical surface area for given basal area and internal volume. A similar model in which the total length of the skeletal elements, and thus the utilization of skeletal material, is the minimizing variable does not generally produce configurations similar to abundant silicoflagellate morphologies. However, the diversity of known silicoflagellate geometries, and especially the occurrence of relatively simple designs such as Corbisema and Dictyocha, indicates that factors other than the simple minimization of apical surface area must also be important in skeletal design.

In both the modern and geologic record, relatively more complex silicoflagellate skeletal configurations occur in high latitudes. In the Recent, six-sided Distephanus tend to predomi- 
Table 11. Abundance of silicoflagellates in selected samples from Hole 697B. Species (in alphabetical order) are recorded as total number of specimens found in slides examined. ${ }^{*}=$ Ebridian.

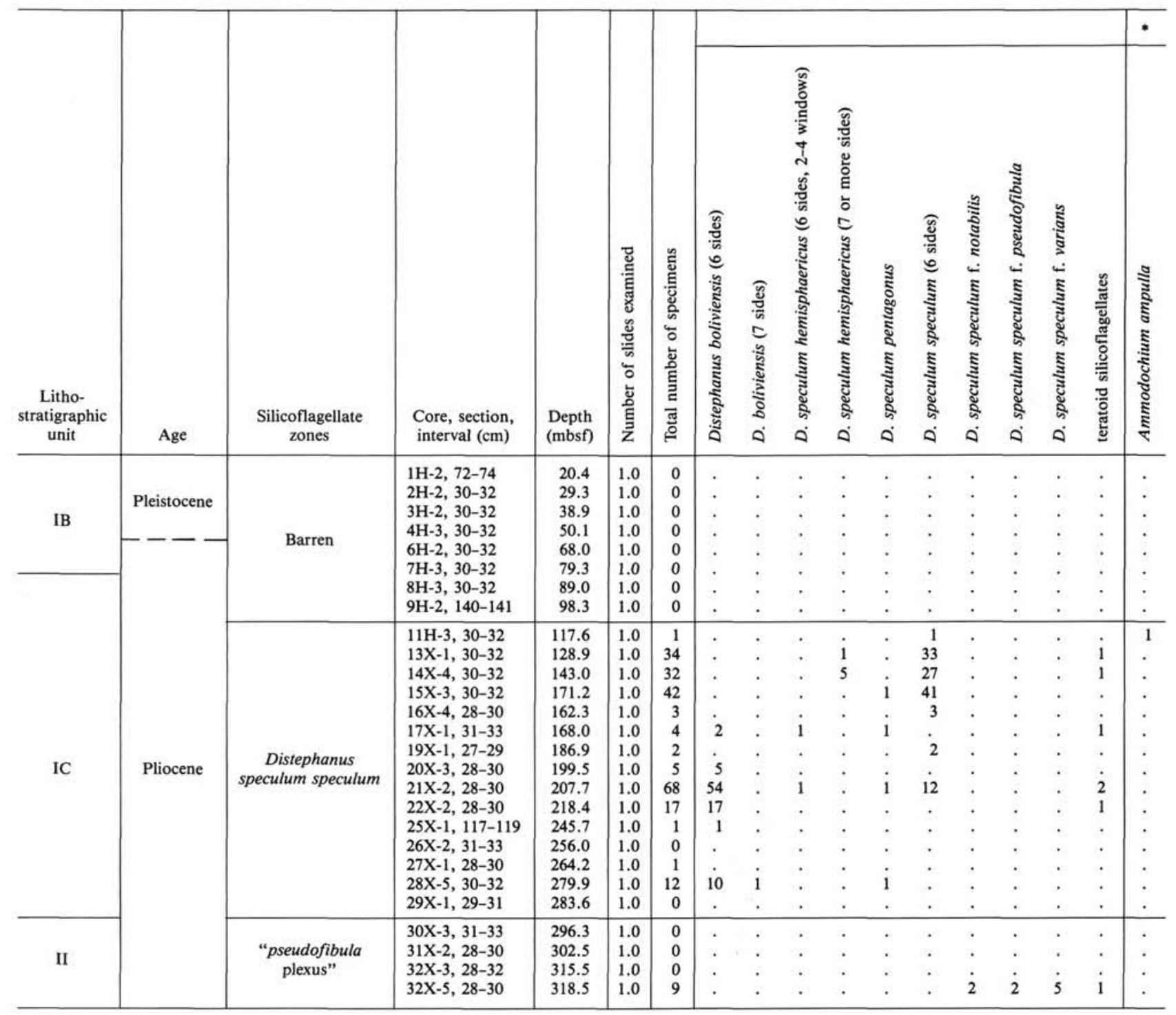

nate in high latitudes while four-sided Dictyocha is dominant in middle and low latitudes. This geographical distribution has led some workers (Yanagisawa, 1943; Poelchau, 1974; Schrader et al., 1986; among others) to propose that the Distephanus/Dictyocha ratio may be useful as a temperature indicator, although Perch-Nielsen (1985) has shown that this may have only regional utility. The dominance of more complex morphologies in high latitudes appears to occur throughout the Cenozoic (McCartney, 1987). For example, the Paleogene high latitudes are dominated by Dictyocha while lower latitudes have a greater abundance of the simpler Corbisema. The greater abundance of Corbisema has led Bukry (1985) to consider this genus an indicator of very warm water. When more advanced skeletal morphologies first appear in the geologic record, they consistently appear in the high latitudes.

The mathematical modeling of silicoflagellate skeletal morphology indicates that more complex morphologies may occur because of a greater need to minimize apical surface area. McCartney and Loper (1989) speculate that the reason for this is the minimization of surface energy, as is the case with soap bub- bles (see Almgren and Taylor, 1976). The occurrence of simpler morphologies in lower latitudes might be due to a need to conserve on the utilization of skeletal material or to limit the weight of the organism. In an environment where both minimization of apical surface area and conservation of silica are important, both Distephanus and Dictyocha will be efficient, though at a different range of internal volume (volume here is measured as a dimensionless ratio, see McCartney and Loper, 1989).

\section{Silicoflagellate Variability}

Silicoflagellate skeletons have been especially noteworthy for their simple geometric configurations and for a sometimes remarkable variability. These qualities have provided ample rationale for both "lumpers" and "splitters" among taxonomists. Silicoflagellate biologists (Van Valkenburg, 1970, 1980) generally belong to the first category and believe that a wide variety of silicoflagellate skeletal types can represent a single species. Silicoflagellate paleontologists, on the other hand, tend to subdivide the taxa because of potential biostratigraphic utility. Thus the taxonomy used by biologists often does not cover in detail 


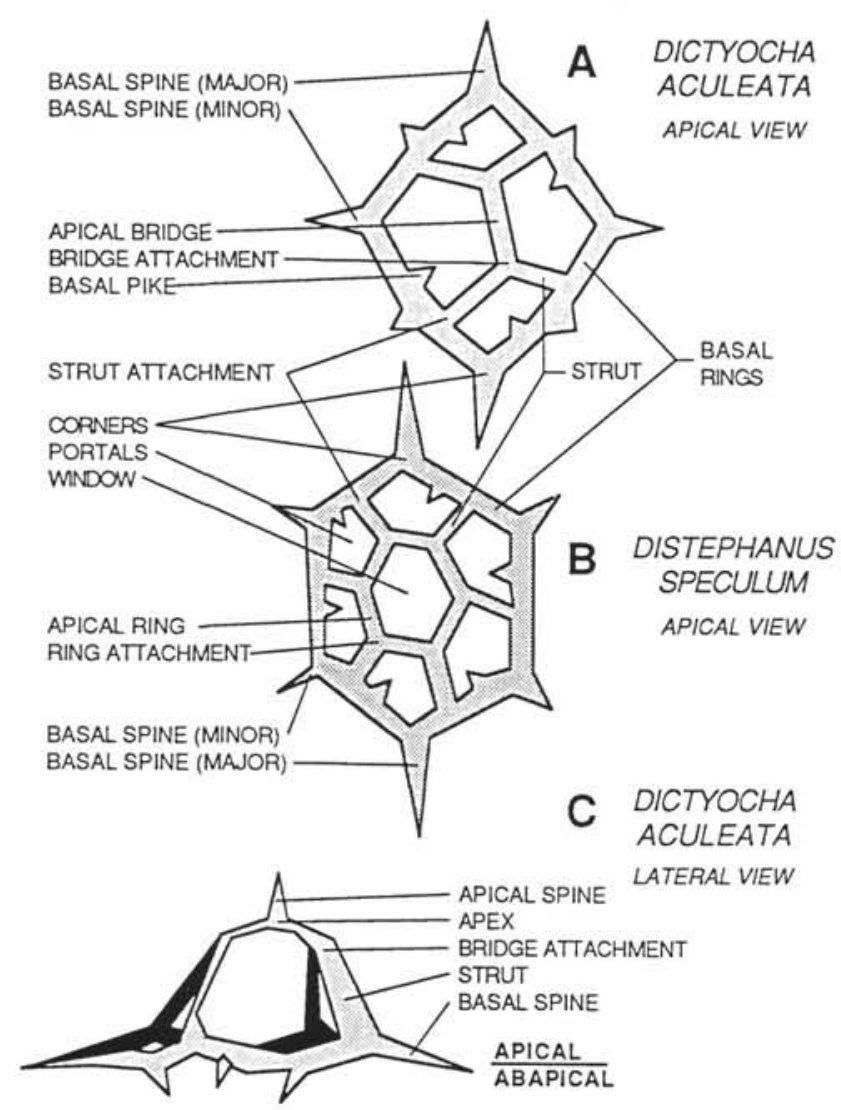

Figure 3. Silicoflagellate descriptive terminology (drawings after Poelchau, 1976). A, C. Apical and lateral views of Dictyocha aculeata (equals $D$. messanensis and D. mandrai of other workers). B. Apical view of Distephanus speculum.

the various skeletal morphotypes, whereas the paleontologic taxonomy covers the relative abundance of the morphotypes in great detail but tends to classify each as a separate taxon.

There is abundant evidence that a silicoflagellate taxonomy based on skeletal design may not show true biological relationships. This evidence consists of multiple skeletal morphologies that are produced by what appears to be a single silicoflagellate species. Six lines of evidence are listed below and then discussed briefly:

1. Cloned cultures of living Dictyocha fibula.

2. Variation in modern populations.

3. Variation between members of a paired skeletal set.

4. Teratoid specimens.

5. Intrataxa gradations. ord.

6. Reoccurrence of some morphologies in the geologic rec-

Perhaps the most oft-cited example of silicoflagellate variability is the study of Van Valkenburg and Norris (1970). In this study, a single cloned culture of Dictyocha fibula produced skeletal morphologies that, if classified by the usual silicoflagellate taxonomy, represent three genera. But although these results give a clear indication that silicoflagellates are capable of extreme variability, it is uncertain how these results should be applied to silicoflagellate taxonomy, as natural populations do not show the variability produced in culture. A large majority of the silicoflagellate skeletons raised by Van Valkenburg are aberrant (e.g., Van Valkenburg and Norris, 1970, fig. 12), and some are almost unrecognizable as silicoflagellate skeletons (Van Valkenburg, 1980, fig. 1).

While exceptional variability does occur in natural populations (Frenguelli, 1935; Van der Spoel et al., 1973; Shitanaka, 1983), most modern populations, especially in the open sea, exhibit only a limited variability. But variability still occurs; for example, Boney (1973) showed that while $95 \%-98 \%$ of Distephanus speculum collected in the Firth of Clyde had hexagonal symmetry, there were also 5-, 7-, and 8-sided specimens. Hovasse (1932c) records a case in which $70 \%-76 \%$ of Distephanus octonarius had octagonal symmetry, with the remainder having $6,7,9$, or 10 sides. Such variability is also found frequently in the fossil record where, for example, a sample with predominantly hexagonal $D$. speculum will have 5 - and 7 -sided specimens associated with it, or where a sample of 4-sided Dictyocha may also have 5 -sided or distephanid variants.

Additional evidence of genetic variability comes from the study of double skeletons. These consist of paired silicoflagellate skeletons connected at the abapical face; they apparently represent organisms that have died during vegetative division. Although paired skeletons are usually very similar, variation sometimes can be considerable. Paired skeletons representing two species or even genera by some interpretations of the silicoflagellate taxonomy have been found by Gemeinhardt (1930) and Bukry and Foster (1973). Boney (1976, 1981), however, notes that such pairings are unusual.

Teratoid (aberrant) silicoflagellate skeletons also give some insight into silicoflagellate variability and show that deviations from the regular morphology may not be lethal to the organism. While they usually make up less than $3 \%$ of a sample (Martini, 1977), they can at times be exceptionally abundant, and even predominant. Common abnormalities include a disruption in the regular symmetry of the skeleton, failure to close the basal or apical ring, and struts that are forked, fused, or occur two to a basal side. Typical teratoids from recent populations are illustrated in some detail for Dictyocha messanensis (Frenguelli, 1935) and Distephanus speculum (Frenguelli, 1938; Boney, 1973; Van der Spoel et al., 1973). Unusual numbers of exceptionally grotesque specimens are sometimes found, such as a sample collected off the northern California coast and described by Poelchau (1974). Aberrants appear to be less common in the open sea than in coastal areas (Bukry, 1978c). Explanations for unusual variation include high chlorinity (Shitanaka, 1983), phytoplankton bloom conditions (Mandra, 1968), genetic weaknesses (Van Valkenburg and Norris, 1971) or other abnormal ecologic conditions (Poelchau, 1974). Because the relative abundance of aberrant specimens may be an indicator of environmental stress, a count of aberrant specimens is included in the abundance tabulations in this chapter.

An additional clue to silicoflagellate variability and the biological relationship between taxa is the occasional occurrence of morphologies intermediate between what are described as separate species or genera. These include naviculopsids with a corbisemid apical ring (Perch-Nielsen, 1975c), corbisemids with a naviculopsid or dictyochid morphology (Dumitrica, 1973; PerchNielsen, 1975c; Bukry, 1975a, 1976a; Busen and Wise, 1977), strutted naviculopsids (Perch-Nielsen, 1976; Bukry, 1976c) and corbisemids with an apical ring (McCartney and Wise, 1987). It is difficult to derive an explanation for these; they could just be aberrants. But these specimens are not otherwise malformed, and where present they occur in some abundance. They may provide insight into the biologic relationships between separate skeletal morphologies and may indicate that distinct morphologies are closely related.

The last line of evidence cited is the recurrence of certain morphologies in the geologic record, particularly some of those considered here with the upper Miocene-lower Pliocene pseudo- 
K. MCCARTNEY, S. W. WISE, JR.

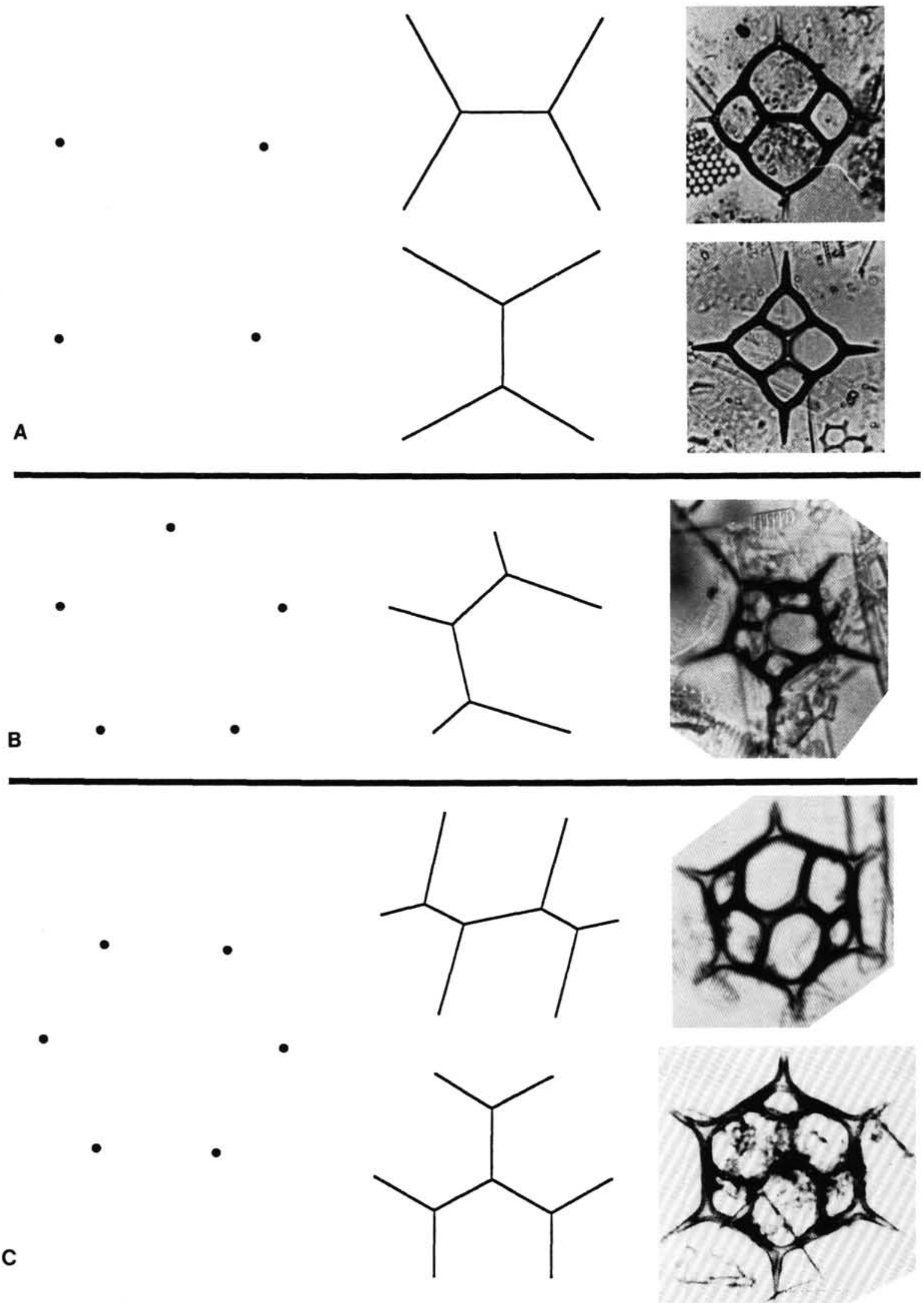

Figure 4. Illustration of optimization and its application to silicoflagellates. The first column shows points positioned at the corners of a square (A), pentagon (B), and hexagon (C) with sides of equal length. The second column shows the minimum-distance configuration that connects all points (from Almgren and Taylor, 1976; Isenberg, 1976). The third column shows dictyochid silicoflagellates with similar apical structures. 
fibula plexus. As discussed below, some of these morphologies co-occur in narrow intervals of the Paleocene (Shaw and Ciesielski, 1983) and Eocene (Locker and Martini, 1987). As evolution should not repeat itself, serious consideration must be given to environmental adaptations for such occurrences. The seeming contradictions of extreme variability in some circumstances and uniformity in others suggest two alternatives. Unusual morphologies could represent ecophenotypic variation. On the other hand, such variation could be caused by genetic exchange between species, which is quite common in plants (see Burger (1975) and Van Valen (1976) and their discussions on oak).

While silicoflagellates are not known to reproduce sexually, Locker and Martini $(1986,1987)$ believe, and we concur, that the great stability of the fossil and recent forms, given their known variability, indicates that silicoflagellates possess temporary phases of sexual reproduction. Thus, a more detailed study of silicoflagellate variability and the more unusual morphologies may help answer questions concerning the silicoflagellate species concept and the biological relationship between the skeletal morphologies.

\section{The Occurrence of the "pseudofibula plexus" of Distephanus speculum}

An exceptionally variable assemblage of six-sided silicoflagellates was found in the upper Miocene/lower Pliocene of most Leg 113 sites. These silicoflagellates generally did not possess an apical ring but instead had a variety of bridge configurations (see Fig. 5). This assemblage, here referred to as the "pseudofibula plexus," apparently represents morphotypes of Distephanus speculum speculum that probably developed in response to some type of ecological stress. Members of this group have been illustrated many times in the literature, but their relationships to one another, and to Distephanus speculum speculum, have not been discussed in much detail. These relationships are important because they show the considerable skeletal variability of the silicoflagellate species and also illustrate how much the skeletal morphology might be affected by changing ecological conditions.

Members of the pseudofibula plexus have been previously reported at high latitudes (Bachmann and Ichikawa, 1962; Ling, 1972; Bukry, 1973, 1975b, 1976a, 1979, 1983; Ciesielski, 1975; Barron, 1976; Shaw and Ciesielski, 1983; Locker and Martini,
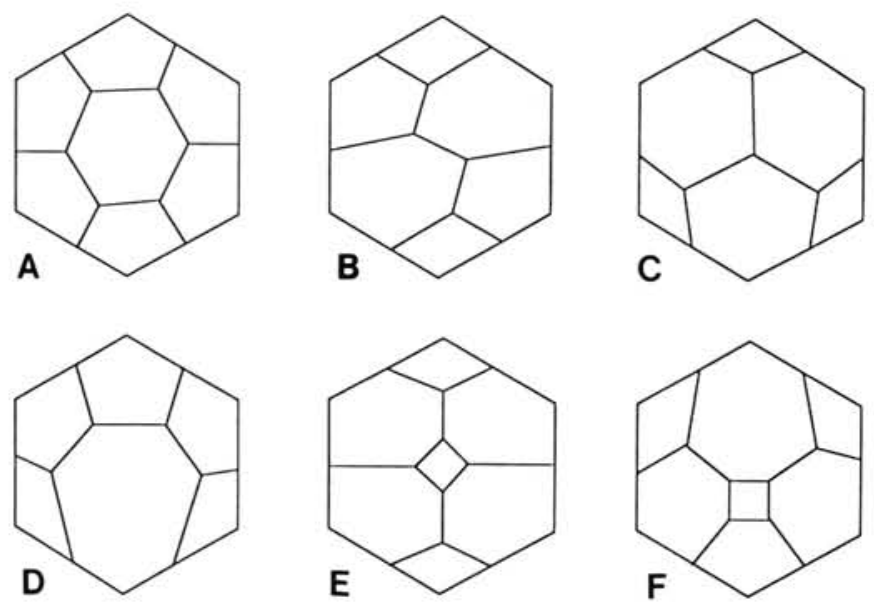

Figure 5. Apical structures of silicoflagellate skeletal morphologies with six basal sides. A. Distephanus speculum speculum. B. f. pseudofibula. C. f. varians. D. f. notabilis. E. f. pseudocrux (centered ring). F. f. pseudocrux (centered ring).
1986) but there has been little effort to show the variability and relative abundance of the morphologies. They have been classified under a variety of species, subspecies, or form names including pseudocrux, pseudofibula, japonica, and varians, although it is generally obvious from the illustrations that a number of distinct skeletal varieties are lumped into each taxon. The different morphologies do not appear to have contrasting stratigraphic ranges, and in fact appear to occur collectively and in close association with Distephanus speculum speculum.

The group is unusual for a number of reasons. To begin with, these silicoflagellates take on what is commonly called a "dictyochid" (apical bridge) morphology, although it appears that they are variants of Distephanus speculum, which of course has an apical ring. While the pseudofibula plexus discussed here is for the most part restricted to the Neogene, similar morphologies have been found elsewhere in the geologic record (PerchNielsen, 1975b; Ling, 1977; Shaw and Ciesielski, 1983) and have been consistently placed taxonomically in Dictyocha. The occurrence reported by Shaw and Ciesielski predates the first appearance of Distephanus. Thus the general morphologies appear to be polyphyletic.

The pseudofibula plexus is both abundant and well-preserved in the lower Pliocene of Leg 113 sites. This assemblage has been used in the Southern Ocean to delineate or help delineate a biostratigraphic zone by Ciesielski (1974, 1975), Bukry (1975c), and Shaw and Ciesielski (1983), and a broad concept of that zone is used in this chapter. As argued below, there is good evidence, however, that it is a group of ecophenotypes, and thus its first and last occurrences should not be viewed as necessarily being time-synchronous. Members of this assemblage have been found within a relatively narrow interval in the uppermost Miocene/lowermost Pliocene of the high southern latitudes (Bukry, 1976a; Ciesielski, 1975; Shaw and Ciesielski, 1983) and confined well within the upper Miocene of low to mid-latitudes elsewhere (Deflandre, 1950; Ling, 1972; Bukry, 1973; Barron, 1976).

Members of the pseudofibula plexus are also found in the Recent Bay of Fundy (Gran and Braarud, 1935), coastal Japan (Tsumura, 1963), and within the atrial contents of Chalina sponges dredged off coastal Argentina (Frenguelli, 1935). Such occurrences in modern coastal waters may be the result of environmental stress, such as reduced salinity. The unusual morphologies may then represent teratoid Distephanus speculum. However, despite their variability, members of this group are relatively consistent in the forms they take, having only three common variants and two others that are less common. The specimens examined from Leg 113 do not have an unusual number of normal silicoflagellate teratologies such as fused or forked spines, two struts connected to a basal side, or distorted basal ring.

Thus it would appear that the unusual assemblage of Distephanus speculum morphologies represented by the pseudofibula plexus is governed by some adaptive advantage under environmental stress. The adaptive advantage itself is unknown but might be related to salinity, water clarity, or some nutrient abundance.

\section{Stratigraphy and Paleoenvironmental Relationships of the "pseudofibula plexus"}

\section{Observations}

In Figure 6, we plot the occurrence of the pseudofibula plexus against lithology at the Leg 113 sites. By this plot we hope to gain some insight into the cause of variation within this group. Included in the figure are the occurrence of dropstones, ice-rafted debris (IRD), and siliceous microfossils as given in the core barrel sheets for each site (Barker, Kennett, et al., 1988). Generally, only dropstones $0.3-0.5 \mathrm{~cm}$ were recorded in the 


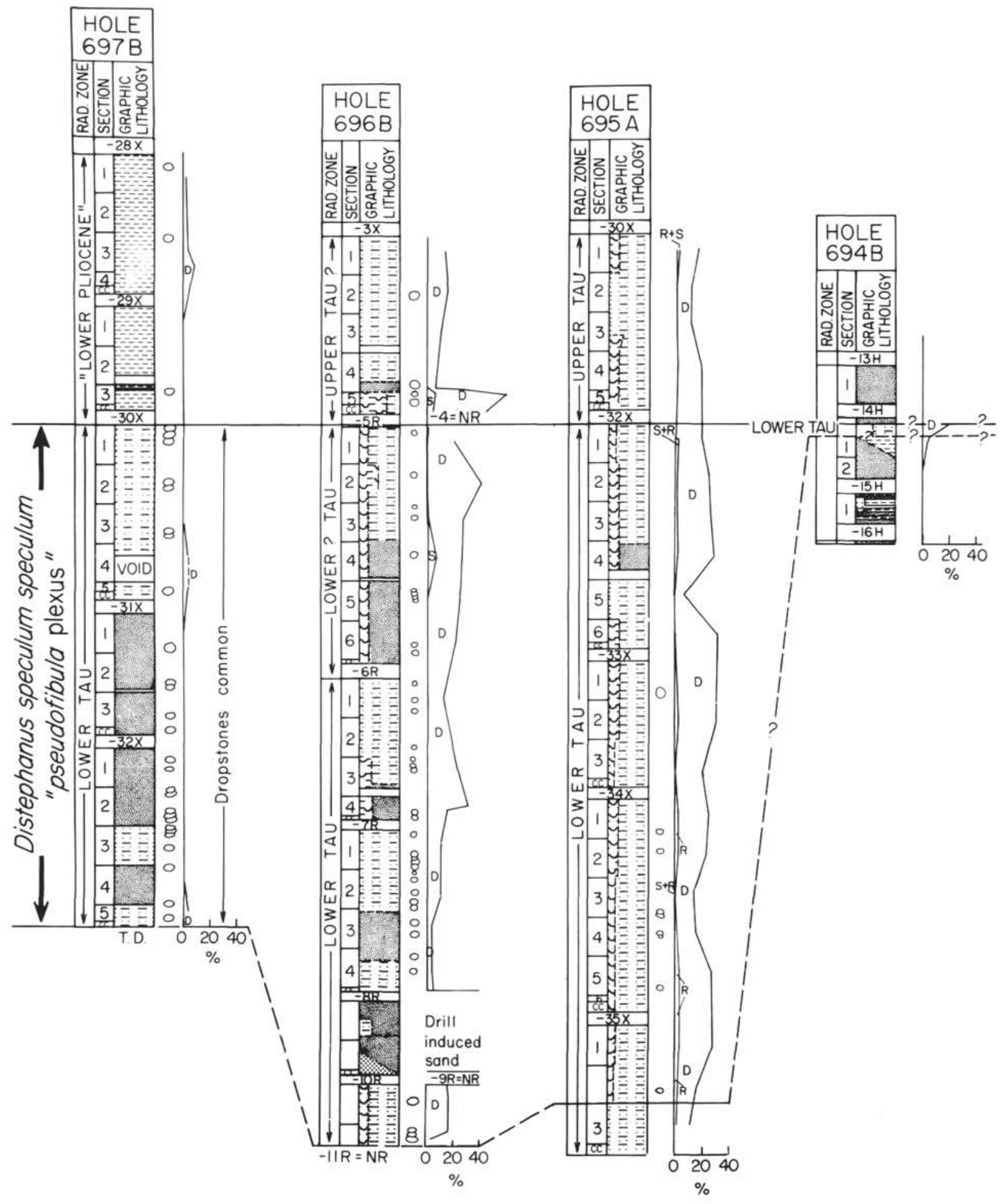

Figure 6. Correlation of the "pseudofibula plexus" among ODP Sites 689-696. Also shown are the occurrences of ice-rafted debris, dropstones larger than $0.3 \mathrm{~cm}$, and siliceous microfossils at each site. Radiolarian zones are from Lazarus (this volume). $\mathrm{D}=$ diatoms; $\mathrm{S}=$ silicoflagellates; $\mathrm{R}=$ radiolarians; irregular circles $=$ dropstones; $\mathrm{NR}=$ no recovery; $\mathrm{T} . \mathrm{D} .=$ total depth; $\sim=$ disconformity. 


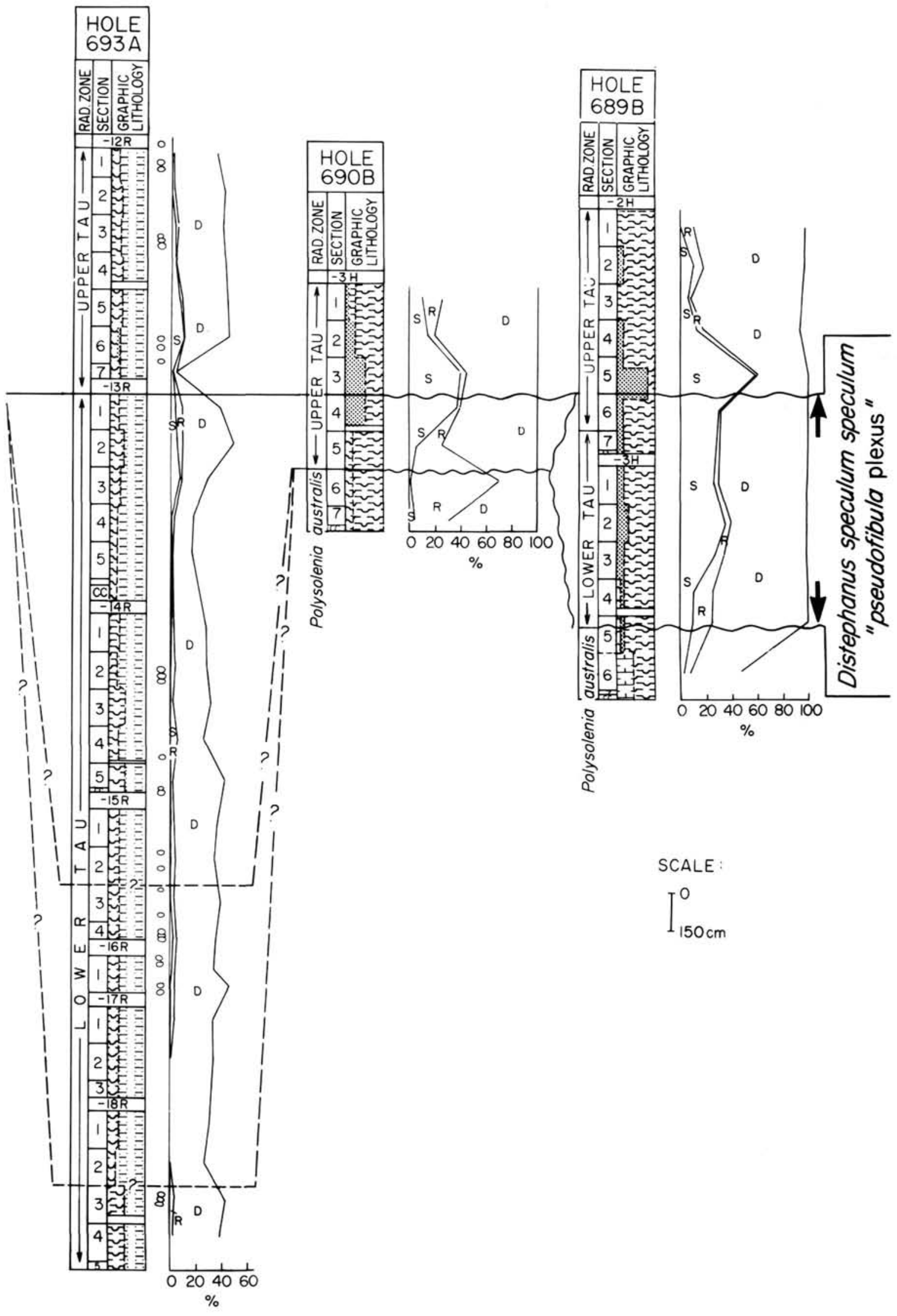

Figure 6 (continued). 
barrel sheets. The radiolarian zones determined for these cores are also given according to the barrel sheets or as updated by Lazarus (this volume, chapter 41).

An examination of Figure 6 shows that at these sites the pseudofibula plexus occurs within the radiolarian Tau Zone, which is late Miocene to early Pliocene in age (4.4-8.0 Ma) according to Lazarus (this volume), but which may extend into the latest Miocene to about $7.9 \mathrm{Ma}$, according to the calibration of Gersonde and Burckle (this volume; see discussion by Gersonde et al., this volume). In the western portion of the study area, the occurrence of the pseudofibula plexus seems to coincide with the last phases of late Miocene-early Pliocene glaciation, as indicated by the occurrence of dropstones and ice-rafted debris at Sites 694 to 697 (Fig. 6; Barker, Kennett, et al., 1988). This glaciation is considered to have encompassed West as well as East Antarctica, and therefore would have included a glaciated Antarctic Peninsula (e.g., Mercer, 1978). Starting with the northeastern sites in the vicinity of the South Orkney Microcontinent (SOM), there seems to be a strong correlation between the occurrence of dropstones and IRD with the occurrence of the "pseudofibula plexus." At Hole 697B, dropstones and IRD are common in Cores 113-697B-30X to 113-697B-32X, but only sporadic immediately above that level, where the numbers of diatoms increase dramatically. Unfortunately the hole had to be terminated due to time constraints before penetrating completely through the pseudofibula plexus.

At Sites 695 and 696 just to the east on the SOM, the same general relationships with IRD and dropstones hold true, but the holes did penetrate deeper. At both sites, no silicoflagellates were present for several cores below the zone. According to smear slide data given in the barrel sheets, diatoms and other siliceous microfossils drop sharply in number below Core 113695A-36X. The same seems to be true for Hole 696B, but this is difficult to demonstrate because of poor recovery, a situation which may have been caused or exacerbated by the presence of numerous dropstones in this portion of the section.

Nevertheless, the absence of silicoflagellates and the diminution in the numbers of siliceous fossils in cores immediately below the pseudofibula plexus indicate diminished siliceous plankton productivity or dilution of the biogenic component by high siliciclastic input. This could have resulted from increased seasonal ice cover in this area or increased input of glacial-marine sediments, either of which would reflect heightened glacial activity prior to the accumulation of silicoflagellates of the pseudofibula plexus.

The only diatom ooze plotted in Figure 6 for these northern sites is in Hole 696B at the base of Core 113-696B-3R, which is the first core recovered above the pseudofibula plexus (there was no recovery in Core 113-696B-4R). Although silicoflagellates increase to $4 \%$ in Section 113-696B-3R-5, no members of the pseudofibula plexus are present, indicating their disappearance once glacial conditions ameliorated and normal siliceous productivity was reestablished.

Site 694, an abyssal site in deep water, characteristically yielded few Pliocene microfossils due to the effects of dissolution at such depths in these latitudes. Recovery in the Pliocene was likewise poor due to the inability to recover poorly consolidated sand turbidites, which constituted most of the lower Pliocene section. Silicoflagellates were recovered only from the lowermost Pliocene. These underlay the bulk of the sand turbidites and belong to the pseudofibula plexus.

At Site 693 on the slope off the coast of East Antarctica, dropstones are common throughout the upper Miocene-Pliocene section, but recovery across the Miocene/Pliocene boundary was poor, apparently due to a high number of dropstones which impeded the coring operation. This site differs from previous ones discussed from Figure 6 in that diatoms, and to some extent silicoflagellates, are more numerous and consistently present throughout the cores. The large number of dropstones would reflect, in part, the proximity of this site to glacial sources and its presence along the coastal iceberg track.

Although truncated by hiatuses, the pseudofibula plexus is well developed at Sites 689 and 690 on Maud Rise, but little correlation can be made there with glacial marine sediments because these sites are virtually devoid of IRD and dropstones. These sites were not situated along any prominent iceberg tracks but instead lay in the path of a warm return current of the Weddell Gyre flowing across the rise from the north or northeast, much as today (Fig. 1). Data collected from Leg 113 sites, particularly the sequences from the SOM, indicate that the pseudofibula plexus developed in this region during or toward the close of the intense late Miocene-earliest Pliocene "West Antarctic" glaciations. This intense glacial episode affected the entire study area but is more evident in Leg 113 sites that lay in the path of the coastal current and the iceberg tracks through the central and western Weddell Sea (Fig. 1). As glacial conditions began to wane during the earliest Pliocene, the pseudofibula plexus disappeared although the general population of siliceous microfossils increased. Accompanying its disappearance was the intense deposition of sand turbidites in the central Weddell Sea, which continued throughout the remainder of the early Pliocene.

\section{Discussion}

The pseudofibula plexus in the study area seems to have developed toward the end of or shortly following the late Mioceneearly Pliocene Antarctic glaciations, the most intense Cenozoic glaciations known until that time (for reviews, see Kennett, 1978; Wise, 1981; and Wise et al., 1985). This glacial interval was followed immediately during the early Pliocene by an exceptionally strong warming, which may have destabilized and even destroyed much of the accumulated ice sheet (Ciesielski and Weaver, 1974; Wise, 1981; and Wise et al., 1985). These late Miocene-early Pliocene glaciations marked one of the most important transitions in Tertiary Earth history. Although the intense glaciations may have begun as early as Tortonian times (Ciesielski and Weaver, 1983), those of the latest Miocene appear to have coincided with the Messinian salinity crisis. The climate of the Antarctic region during these late Miocene-early Pliocene glacial episodes would have been in a state of flux, experiencing sharp changes in temperature, upwelling, surface water salinity and nutrient supply, and the distributions of water masses, land, and sea ice.

We speculate that the pseudofibula plexus developed from the long-ranging Distephanus speculum speculum stock as a response to these strong environmental changes. We believe the morphologic variability within the plexus records an ecophenotypic response to any one or a combination of factors such as reduced salinity, change in nutrient supply, upwelling, or rapidly changing temperatures, although it is impossible at this writing to specify what the most important factor(s) would have been. In support of this speculation, we note that the plexus was relatively short-lived in this region, and that several of the variations have been seen at least once previously but at different times in the geologic record of other regions. We see no link between the forms we observed in our Southern Ocean cores and the pseudofibulids described from the upper Miocene of the North Pacific. We therefore follow Schulz (1928) and Locker and Martini (1987) in regarding the various morphotypes as forms rather than species or even subspecies.

Of the several possible environmental factors that may have produced the stress or change that led to these variations, we consider a reduction in salinity one of the more likely. Any regional reductions in salinity could have amplified the residual effects of the Messinian salinity crises of the latest Miocene, 
which removed a considerable amount of salt from the world's oceans. If interglacial conditions were at all similar to today during the late Miocene-early Pliocene glaciations, then the existence of an equivalent to the present-day Antarctic Surface Water Mass would have produced a photic zone in our study area characterized by very cold temperatures and reduced salinities (see Wise, 1981, fig. 2). Eventual massive melting of the ice sheets (deglaciation) following the glaciations would have done the same. Strongly fluctuating glacial-interglacial conditions leading up to or accompanying such a deglaciation may have also accounted for the extensive sand turbidites seen at Site 694. Although reduced salinities in the Antarctic Surface Water Mass might account for the development of the latest Miocene-early Pliocene pseudofibula plexus around Antarctica, this would not necessarily explain the presence of the plexus elsewhere in the world's oceans, such as during the late Miocene in the North Pacific (e.g., Bukry, 1973).

As indicated previously, other factors may have induced the variability seen in the pseudofibula plexus. It is possible that the global oceans could have experienced changes in upwelling rates during the late Miocene-early Pliocene glacial episodes. This would have affected the supply of nutrients, both the kind and amount. Silica, for example, is an important and sometimes limiting nutrient. The double-ringed morphology of Distephanus speculum speculum does not make minimal use of skeletal material, while the various pseudofibulid morphotypes are more silica efficient (Fig. 4). The latter, then, could be an adaptation to silica-poor nutrient conditions. This is opposed to the normal trend for Distephanus speculum, which favors skeletal structures that minimize surface area, as discussed previously in this chapter. Minimal surface area might be more favored by the species in times of exceptionally nutrient-rich upwelling where little surface area is needed to absorb the necessary nutrients and silica is sufficiently plentiful to allow less silica efficient skeletal constructions. If this were the case, the pseudofibulid condition would be favored during deglaciations and long interglacials, whereas the normal Distephanus speculum speculum morphology would characterize predominantly glacial times.

Regardless of what induced the pseudofibulid condition, $\mathrm{Di}$ stephanus speculum rather quickly adapted to the predominantly glacial climates that followed the early Pliocene deglaciation. Throughout the remainder of the Pliocene-Pleistocene Epochs, which were predominantly glacial, $D$. speculum speculum and/or the closely allied double-ringed $D$. boliviensis were the overwhelmingly dominant high-latitude silicoflagellate taxa, just as $D$. speculum speculum is in these latitudes today (see range charts).

\section{SYSTEMATIC PALEONTOLOGY}

The synonymies include only the first description and, if needed, a recent reference that has a more complete taxonomy.

\section{Silicoflagellates}

Genus BACHMANNOCENA Locker, 1974 emend. Bukry, 1987

Introductory remarks. The Mesocena group is exceptionally diverse and contains a variety of forms that may not be closely related. Some workers (Tappan, 1980; McCartney, 1987) believe that some members of this group are ecophenotypic variants of silicoflagellates that possess apical structures. Other workers (Locker and Martini, 1986) have subdivided this group into a number of different genera. Thus, it is perhaps fortuitous that Bukry (1987) has, on taxonomic grounds, proposed the genus name Bachmannocena for all silicoflagellates that have a basal ring without an apical structure. This gives us a new chance to build a useful taxonomy without the ever-more-complex trappings of the old. The authors hope that this new usage will continue until we have a much better understanding of this enigmatic group.

\section{Bachmannocena apiculata (Schulz)}

(Plate 2, Figs. 6-10)

Mesocena oamaruensis apiculata Schulz, 1928, p. 240, fig 11.

Mesocena apiculata (Schulz) Ling, 1972, p. 173, pl. 28, figs. 2-4. Bachmannocena apiculata (Schulz) Bukry, 1987, p. 403-404.

Remarks. Bachmannocena apiculata is a relatively large $(55 \mathrm{~m})$, three-sided form with short spines. There is wide variation in the shape of the ring, as shown by the illustrated specimens. The basal ring is in some cases equilateral and in others more elongate; the two longer basal sides of elongate morphologies are often curved. This taxon was not subdivided into multiple subspecies (Bukry, 1987) because the various morphologies occurred together and, at least in this study, did not appear to have any biostratigraphic significance.

\section{Bachmannocena circulus (Ehrenberg)}

Mesocena circulus (Ehrenberg) Ehrenberg, 1844, p. 65. Bachmannocena circulus (Ehrenberg) Bukry, 1987, p. 404.

Remarks. This species was found only in Sample 113-689B-6H-2, $29-31 \mathrm{~cm}$, in which it was dominant.

\section{Bachmannocena diodon (Ehrenberg)}

Mesocena diodon Ehrenberg, 1844, p. 71, 84.

Bachmannocena diodon (Ehrenberg) Bukry, 1987, p. 404.

Remarks. Teratoid forms of this taxon were not found. This contrasts considerably with Mesocena diodon discussed in McCartney and Wise (1987).

\section{Bachmannocena occidentalis (Hanna ex Bukry), n. comb.}

Mesocena oamaruensis var. quadrangula Schulz, 1928 (in part), p. 240, fig. 12.

Mesocena occidentalis Hanna, 1931, p. 200, pl. E, fig. 1.

Mesocena occidentalis Hanna ex Bukry, 1977, p. 832.

Remarks. Four-sided Bachmannocena occidentalis were found only in Section 113-689B-13H-2, 29-31 cm.

Genus CORBISEMA Hanna, 1928

Corbisema apiculata (Lemmermann)

Dictyocha triacantha var. apiculata Lemmermann, 1901, p. 259, pl. 10, figs. $19,20$.

Corbisema apiculata (Lemmermann) Ling, 1972, p. 153, pl. 24, fig. 1.

Remarks. This is a generally middle-sized $(40 \mu \mathrm{m})$ Corbisema with relatively robust basal ring and basal spines of moderate length and large sustaining spines. One of the basal sides is usually shorter than the other two, giving the basal ring a slightly elongate shape. Specimens were relatively uncommon in the Oligocene of Hole 689B.

\section{Corbisema hastata (Lemmermann)}

Corbisema triacantha var. hastata Lemmermann, 1901, p. 259, pl. 10, fig. $16,17$.

Corbisema hastata (Lemmermann), Ling, 1972, p. 155, pl. 24, fig. 5.

Remarks. The primary characteristic of this species is a basal ring with one side shorter, and often much shorter, than either of the other two sides. This usually gives the basal ring an "arrowhead" shape. This group has considerable variation and some workers (see Bukry, 1976a) have subdivided it into many subspecies. Since the occurrence of $C$. hastata in this study was sporadic and restricted to Site 689 , no effort has been made here to subdivide the specimens below the species level.

\section{Corbisema inermis inermis (Lemmermann)}

Dictyocha triacantha var. inermis Lemmermann, 1901, p. 259, pl. 10, fig. 21.

Corbisema inermis inermis (Lemmermann) Bukry, 1976a, p. 892.

Remarks. This species has a large basal ring that is relatively fragile in appearance and generally lacks basal spines. The sides are usually close to equilateral. There is much variation in the degree to which the basal sides bow outward, and thus the shape of the corners. The specimens in this study generally have relatively linear sides and sharp cor- 
ners, although some specimens have very rounded corners similar to that of $C$. geometrica (but without the apical plate).

\section{Corbisema triacantha (Ehrenberg)} (Plate 1, Fig. 1)

Dictyocha triacantha Ehrenberg, 1844, p. 80.

Corbisema triacantha (Ehrenberg) Busen and Wise, 1977, p. 713.

Remarks. The variation in this taxon was very similar to that found by McCartney and Wise (1987). While several subspecies are recognized (Bukry, 1977, 1978a; Ling, 1972), the specimens observed in this study did not fall into distinct taxa and thus were considered collectively.

\section{Genus DICTYOCHA Ehrenberg, 1837}

Dictyocha aspera (Lemmermann)

(Plate 2, Fig. 2)

Dictyocha fibula var. aspera Lemmermann, 1901, p. 260, pl. 10, figs. $27,28$.

Remarks. Any Dictyocha in which the apical bridge was parallel to the minor axis were counted in this study as $D$. aspera. This morphology occurred sporadically in Holes 689B and 690B. Asperid specimens generally had a relative short bridge and frequently showed a close similarity to associated $D$. fibula.

\section{Dictyocha byronalis Bukry}

Dictyocha byronalis Bukry in Barron et al., 1984, p. 151, pl. 3, figs. 14.

Remarks. This taxon has a slightly elongate basal ring and an apical bridge that is rotated sinistrally about $10^{\circ}$ (when seen from apical view).

\section{Dictyocha calida Poelchau}

(Plate 2, Fig. 4)

Dictyocha calida Poelchau, 1976, p. 169, pl. 1, figs. c, d; pl. 3, figs. a-f.

Remarks. This species has a basal ring whose corners very nearly delineate a square. Basal spines are usually of equal length. The bridge is generally very short. Poelchau (1976) has shown that this species is commonly found, but is seldom dominant, in the modern North Pacific. In this study it was found only in Section 113-689B-5H-5, where it is relatively abundant.

\section{Dictyocha deflandrei Frenguelli ex Glezer}

(Plate 2, Figs. 5A, 5B)

Dictyocha deflandrei Frenguelli, 1940 (in part), p. 65, figs. 14a, 14c-f. Dictyocha deflandrei Frenguelli ex Glezer, Bukry, 1975c, p. 854, pl. 2, figs. 9-13.

Remarks. Specimens of Dictyocha deflandrei found in Oligocene of Hole 689B were very similar to associate frenguellii but lacked apical spines. This taxon can exhibit considerable variability, with some variants having five or seven basal sides (Bukry, 1975c). A number of subspecies have been described (see Glezer, 1966; Bukry, 1978b) but these are not used in this work. For further comment on this interesting group, see the remarks with frenguellii.

\section{Dictyocha fibula Ehrenberg}

Dictyocha fibula Ehrenberg, 1839, fide Loeblich et al., 1968, p. 90, pl. 9, figs. 9-12.

Dictyocha fibula Ehrenberg, Bukry and Foster, 1973, p. 826-827.

Remarks. The authors use a broad interpretation of this species. The taxa occurs sporadically in Holes 689B and 690B.

\section{Dictyocha frenguellii Deflandre (Plate 2, Fig. 3)}

Dictyocha frenguellii Deflandre, 1950, p. 194, figs. 188-193.

Remarks. Dictyocha deflandrei and $D$. frenguellii form a very interesting group of silicoflagellates. Both are generally similar in size and have robust skeletal elements. In addition, both have unusual apical structures that, unlike most other silicoflagellates, appear to make no concession to conserving skeletal material. Skeletons of $D$. deflandrei have struts which arch upwards to support an apical plate; specimens of
D. frenguellii have a similar apical structure but with long apical spines. The overlapping ranges found in this study and elsewhere (Bukry, 1975a, $1975 \mathrm{c}$ ) indicate a close evolutionary relationship, with $D$. deflandrei being the ancestral form. Other unusual silicoflagellates appear also to belong to this group, including the hexagonal or trigonal $D$. spinosa and D. hexacantha.

The development of long apical spines in D. frenguellii and D. spi$n o s a$, as well as the additional basal spines that occur on Dictyocha hexacantha, is very interesting. In all three cases, the presence of spines appears to violate the rule of triple-junctions (see Poelchau, 1974). In other morphologies where apical spines occur, such as on some Distephanus speculum, the apex of Dictyocha aculeata, and on Naviculopsis trispinosa, the apical spine occurs where two other elements meet at an angle and thus the spine becomes the third member of the junction. On $D$. spinosa and $D$. hexacantha, however, the spine becomes a part of a quadruple junction. On $D$. frenguellii the apical spine does not appear to be located where two elements meet at an angle. The reason why such unusual spines occur, if in fact they do serve a function, is unknown. However, Kitchell (1983) has shown that the effect of silicoflagellate spine length on sinking rate might be considerable and could be a useful indicator of upwelling intensity. Recent experiments with simple models sinking in glycerol of low Reynolds number (Sargeant et al., 1987) indicate that the presence of spines slows the rate of settling, and that settling rate decreases with an increase in spine length. Similar conclusions have been reached with living diatoms (Conway and Trainor, 1972; Walsby and Xypolyta, 1977). The interpretation of this taxon as used by the authors is rather broad and may include specimens of $D$. fischeri (Bukry, 1976a).

\section{Dictyocha pentagona (Schulz)}

(Plate 1, Fig. 8)

Dictyocha fibula var. pentagona Schulz, 1928, p. 255, fig. 41a.

Dictyocha pentagona (Schulz) Bukry and Foster, 1973, p. 827, pl. 3, fig. 10.

Remarks. All silicoflagellate specimens with five basal sides and an apical bridge, except those that appear to be part of the pseudofibula plexus, were counted as $D$. pentagona. While listed here as a separate species, the specimens observed in this study might include variants of both Dictyocha and Distephanus taxa. One group of five-sided dictyochid forms, however, appeared to be part of the pseudofibula plexus and was thus counted as Distephanus speculum speculum f. pseudopentagonus.

\section{Dictyocha spinosa (Deflandre)}

(Plate 2, Fig. 2)

Corbisema spinosa Deflandre, 1950, p. 193, figs. 178-182.

Dictyocha spinosa (Deflandre) Glezer, 1966, p. 238, pl. 10, figs. 6-8.

Remarks. Dictyocha spinosa is an unusual species similar to $D$. hexacantha, but has apical spines that are not in the basal plane. Observed specimens of Dictyocha spinosa were similar in size and structure to associated $D$. frenguellii.

\section{Dictyocha stapedia Haeckel}

Dictyocha stapedia Haeckel, 1887, p. 1561, pl. 101, figs. 10-12.

Remarks. This is a relatively small fibulid form with a long apical bridge that is rotated sinistrally when seen from apical view. It appears to be part of an evolutionary lineage that includes, in more recent strata, Dictyocha subaculeata and $D$. aculeata. Dictyocha aculeata is larger and has an apical bridge that is further rotated. Dictyocha stapedia was occasionally found in the upper Miocene of Hole 689B and 690B.

Genus DISTEPHANUS Stohr, 1880

Distephanus boliviensis (Frenguelli)

(Plate 3, Fig. 4)

Remarks. The six-sided Distephanus exhibit considerable variability. The group is generally subdivided into two species, but the biologic relationship between these is poorly understood. The first of these, Distephanus boliviensis, is a group characterized by a large basal ring and robust skeletal elements whereas morphologies ascribed to Distephanus speculum are smaller and have a more fragile appearance. In many samples the two taxa are distinct and easily separated, but in other samples 
(e.g., 113-690B-3-2, 30-32 cm) they appear to intergrade (it is for this reason that the boliviensis morphologies are not used as a zonal indicator in this study).

Specimens of $D$. boliviensis dealt with in this study generally had long and more-or-less equal spines. However, there is much variation, and some samples were dominated by forms that had two major and four minor spines.

\section{Distephanus boliviensis major (Frenguelli)}

Dictyocha boliviensis Frenguelli, 1940 (in part), p. 44, figs. 4 b-d. Distephanus boliviensis major (Frenguelli), Bukry, 1978a, p. 817.

Remarks. Cannopilid specimens associated with Distephanus boliviensis were found in the upper Miocene and late Pliocene of Hole 689B.

\section{Distephanus crux (Ehrenberg)}

$$
\text { (Plate 3, Figs. 1-2) }
$$

Distephanus crux Ehrenberg, 1840, p. 207; Ehrenberg, 1854, pl. 18, fig. 56, pl. 33, fig. 9.

Remarks. This taxon showed considerable variability, especially in the relative size of the apical ring and the aspect ratio of the basal ring. Specimens with a relatively long major axis were counted as $D$. longispinus, although there appeared to be a close relationship between this form and associated $D$. crux. The forms with a slightly elongated basal ring, however, appear to be useful biostratigraphically.

\section{Distephanus longispinus (Schulz)}

(Plate 3, Fig. 3)

Distephanus crux f. longispinus Schulz, p. 256, fig. 44.

Distephanus longispinus (Schulz), Bukry and Foster, 1973, p. 828, pl. 4, figs. 7, 8 .

Remarks. See remarks following Distephanus crux.

\section{Distephanus speculum hemisphaericus (Ehrenberg)}

(Plate 4, Figs. 1A-B, 3A-B, 4A-B, 5A-B, 6, 7)

Dictyocha hemisphaerica Ehrenberg, 1844, pl. 17, fig. 5.

Distephanus speculum hemisphaericus (Ehrenberg), Bukry, 1975c, p. 854.

Remarks. Distephanus with multiple windows occurred sporadically throughout Hole 689B and are especially abundant in Section 113-689B$7 \mathrm{H}-5$. These showed exceptional variability, from forms with two windows to others that were cannopilid in appearance (see Pl. 4). There was similar variability in the number of basal sides. Since the occurrence of these forms together indicates to the authors a close relationship and since there was no obvious criteria for subdividing this complex group, all forms were counted together, although a distinction is made between those that had up to or more than four windows. The abundant cannopilid morphologies found in the Oligocene of Hole 689B appeared to be closely related to co-occurring Distephanus speculum, but were sometimes larger in size.

\section{Distephanus speculum minutus (Bachmann)}

Dictyocha speculum f. minuta Bachmann in Ichikawa et al., 1967, p. 161.

\section{Distephanus speculum pentagonus Lemmermann}

(Plate 3, Figs. 6A-C, 7, 8A-B)

Distephanus speculum var. pentagonus Lemmermann, 1901, p. 264, pl. 11, fig. 19.

Distephanus speculum pentagonus Lemmermann, Bukry, 1976a, p. 895.

Remarks. The five-sided Distephanus is generally less abundant than associated six-sided forms. However, it occasionally is predominant (Bukry, 1981). In Hole 689B, the pentagonid form is predominant in two widely separated samples. In each case, the association of abundant five-sided Distephanus with other unusual silicoflagellates such as $\mathrm{Na}$ viculopsis trispinosa (Section 113-689B-13H-2) and teratoid specimens (Section 113-689B-5H-5), indicates that the abundant occurrence of this form may be related to some environmental stress.

Two unusual five-sided distephanid forms were found. The first of these (Pl. 3, Fig. 7) has two long basal spines and three smaller ones. The general appearance is of a Distephanus crux on one side of the ma- jor spines and a $D$. speculum on the other; a similar specimen was illustrated by McCartney and Wise, 1987, pl. 2, fig. 9). This form could be a teratoid specimen of either of the above species, or it could indicate that these species are more closely related than is generally believed.

A second unusual five-sided distephanid was represented by four specimens in Sample 113-689B-6H-5. This morphology (PI. 3, Figs. 6, 8 ) has a nearly circular basal ring and a relatively large apical ring supported by struts that are almost perpendicular to the basal plane. The apical ring can be as large as the basal ring.

Distephanus speculum speculum (Ehrenberg)

(Pl. 3, Fig. 5; Pl. 4, Fig. 2A-B; Pl. 6, Fig. 1)

Dictyocha speculum Ehrenberg, 1840; Ehrenberg, 1854, pl. 18, fig. 57; pl. 19, fig. 41 ; pl. 21, fig. 44 ; pl. 22, fig. 47.

Remarks. The six-sided distephanids exhibit considerable variability. This variation is most obvious in the size of the basal ring, the relative diameter of the apical ring, and the length of basal spines, but many other morphologic characters vary as well. There also were seven- and more-sided specimens (see Pl. 4, Fig. 2). These were counted separately to show some of the variability within the sample, but were not given subspecies or forma rank.

\section{The "pseudofibula plexus" of Distephanus speculum speculum}

Introductory remarks. In most sites an upper Miocene/lower Pliocene assemblage of small, six-sided silicoflagellates with an exceptionally variable apical structure was found. This assemblage may represent a group of variants of Distephanus speculum that occur because of some type of ecological stress. They have been previously reported at high-latitude sites. They are combined here into one group with three distinct variants that do not have an apical ring and a less common variant that has an apical ring with fewer sides than the basal ring.

Various workers (Bachmann and Ichikawa, 1962; Martini, 1971; Ling, 1972; Bukry, 1973, 1975b, 1976a, 1979, 1983; Ciesielski, 1975; Dzinoridze et al., 1978; Shaw and Ciesielski, 1983; Locker and Martini, 1986) have previously reported unusual six-sided silicoflagellates from upper Miocene or lower Pliocene high latitude sections that have unusual apical structures. These are usually classified under the species or subspecies names pseudocrux, pseudofibula, japonica or varians, although it is generally obvious from the illustrations that a number of distinct skeletal varieties are lumped into each taxon. The different skeletal morphologies (shown in Fig. 5 of the text) do not appear to have contrasting stratigraphic ranges, and in fact appear to occur collectively. These unusual skeletal morphologies occur in a relatively short interval from a number of the sites of Leg 113. These are here called forma pseudofibula, varians, and notabilis. Another morphology that is characterized by a four- or five-sided apical ring and referred to here as pseudocrux is much less common at Leg 113 sites but appears to be more common elsewhere. The pseudocrux morphology is assumed to be a part of the pseudofibula plexus, but future work on it is needed; Ling (1972) indicates that the pseudocrux morphology may have a slightly different range. A fifth morphotype in this plexus, Distephanus speculum speculum f. pseudopentagonus, is described below as new. At most Leg 113 sites, the assemblage occurs close to the point in the hole where the deposition of abundant upper Miocene-lower Pliocene ice-rafted material ceased.

\section{Distephanus speculum speculum $\mathrm{f}$. pseudofibula Schulz}

(Pl. 5, Figs. 1-4; Pl. 6, Figs. 2, 3)

Distephanus speculum f. pseudofibula Schulz, 1928, p. 262, fig. 51a, b.

Remarks. The apical structure of this morphology consists of a three-element apical bridge that divides the skeletal morphology into similar halves; the bridge bifurcates at each end and at some place between the bifurcations has two struts that attach to opposite sides of the basal ring. The original description (Schulz, 1928) includes only specimens of this type, but some subsequent workers (for example, Ciesielski, 1975; Locker and Martini, 1986) have used this taxon to cover all the morphologies belonging to what is here referred to as the pseudofibula plexus.

Distephanus speculum speculum $\mathrm{f}$. varians Gran and Braarud (Pl. 5, Figs. 8-9, 13 [upper]; Pl. 6, Figs. 4, 6)

Distephanus speculum f. varians Gran and Braarud, 1935, p. 390, fig. $68 \mathrm{~b}$ (not 68a). 
Remarks. This morphology has a six-sided basal ring and an apical structure that is triradially symmetric, with a triple-junction directly above the center of the basal plane. Each member of this triple-junction then bifurcates into two struts that attach to adjacent basal sides. Gran and Braarud (1935), in their original description, illustrate both the triradiate morphology and the asymmetrical morphology discussed below without specifying one of these as the holotype. Subsequent usage has applied the varians term to the triradiate form, and thus we designate figure $68 \mathrm{~b}$ of Gran and Braarud as the lectotype for the varians form.

\section{Distephanus speculum speculum}

f. notabilis, n. stat.

(Pl. 5, Figs. 5, 10-12, 13 [lower]; Pl. 6, Figs. 5, 7)

Distephanus speculum f. varians Gran and Braarud, 1935, p. 390, fig. $68 \mathrm{a}($ not $68 \mathrm{~b})$.

Distephanus speculum varians Gran and Braarud, Bukry, 1976a, pl. 8, fig. 10.

Distephanus speculum notabilis f. notabilis Locker and Martini, 1987, pl. 5, figs. $40-41$.

Remarks. The apical structure of this morphology consists of a three-element apical bridge that is asymmetric with respect to any basal axis. The bridge has a curved shape with all struts connected to the outside of the curve. The two struts connected to opposite ends of this apical bridge are attached to adjacent basal sides. The apical structure thus takes the shape of an incomplete and tilted apical ring. Some specimens were found in which the apical ring was complete but not centered above the middle of the basal ring; these where counted as Distephanus speculum speculum. The occurrence of these types, as well as normal Distephanus speculum speculum of similar size, spine lengths and general construction is evidence that these are varieties of six-sided Distephanus. The form name notabilis is taken from Locker and Martini (1987), who used this taxon as a subspecies among Distephanus speculum morphologies of a much earlier (Eocene) age. The notabilid morphologies described by Locker and Martini are similar to those found in the Miocene/Pliocene pseudofibula plexus, and their subspecies name is used here as a form name for the general morphology. The Locker and Martini paper is important because it shows that the pseudofibula plexus associated with Distephanus speculum occurred elsewhere in the geologic record, presumably when the silicoflagellates were again under some environmental stress. To be consistent with our nomenclature for these forms, we transfer this name to form status.

\section{Distephanus speculum speculum f. pseudocrux (Schulz). (Text Figs. 6e, 6f)}

Distephanus speculum f. pseudocrux Schulz, 1928, p. 263, fig. 52a, b. Dictyocha japonica Deflandre, 1950 (in part), pl. 17, fig. 18. Distephanus pseudocrux (Schulz), Ling, 1977, pl. 2, figs. 16-17.

Remarks. Much less common than the types discussed above were varieties in which some struts bifurcate so that the apical ring has less skeletal elements than the basal ring. In its best-known forms (Fig. 6 $e, f)$, four struts meet to support a four-sided apical ring. Thus the apical structure has altogether eight struts or, alternatively, six struts, a four-element apical ring and two apical bridges. There are two general morphologies. In one, the apical ring is above the center of the basal ring and the extra struts (or apical bridge elements) attach to opposite corners of the apical ring (see Fig. 6e). The long axis of the apical structure is usually, but not always, parallel to the basal major axis. In a second and apparently less common morphology (Fig. 6f), the apical ring may be centered with the extra struts attaching to adjacent apical corners.

\section{Distephanus speculum speculum $\mathrm{f}$. pseudopentagonus $\mathrm{n}$. $\mathrm{f}$.} (Plate 5, Fig. 6)

Diagnosis. Five-sided basal ring with an apical structure similar to that of Distephanus speculum speculum f. pseudofibula.

Description. Five short to moderately long spines extend from the corners of the basal ring. The apical structure consists of two central bar segments which bend at their point of intersection, where they are also joined by a strut. At each of their other ends, the segments bifurcate into two struts. These segments and struts subdivide the central area into five portals of varying size. Basal pikes extend into each portal from the basal ring.
Occurrence. Lower Pliocene of ODP Sites 689, 690, and 693 .

Size. Holotype, $45 \mu \mathrm{m}$.

Holotype. Plate 5, Figure 6.

Type locality. ODP Sample 113-689B-2H-2, 33-35 cm.

Remarks. Among the variety of five-sided dictyochid forms observed in this study were skeletons that appeared to be part of the pseudofibula plexus, and are thus related more closely to Distephanus speculum speculum than to any Dictyocha. These were tabulated separately on the range charts in order to show the variability within this interesting group. Specimens counted in this group tended to be smaller than those counted as Distephanus speculum pentagonus.

\section{Genus NAVICULOPSIS Frenguelli, 1940 \\ Naviculopsis constricta (Schulz)}

(Plate 1, Figs. 6, 7)

Dictyocha navicula biapiculata constricta Schulz, 1928, p. 246, fig. 21. Naviculopsis constricta (Schulz), Bukry, 1975c, p. 856.

Remarks. This species has a relatively low band with elliptical portals. It was uncommonly found near the bottom of Hole 689B.

\section{Naviculopsis eobiapiculata Bukry}

(Plate 1, Figs. 2-4)

Naviculopsis eobiapiculata Bukry, 1978b, p. 878.

Remarks. This taxon has a highly curved band that is of constant width until the band is close to the basal ring. While easily distinguished from $N$. constricta when slightly tilted (see fig. 2 of McCartney and Wise, 1987), identification can be difficult when the specimen lies flat upon its basal ring.

Many of the specimens found in Cores 113-689B-8H to 113-689B$13 \mathrm{H}$ have a basal ring similar to $N$. biapiculata but with basal spines that are two-thirds the length of the basal ring. True $N$. biapiculata, according to Bukry (1978b), would have spines that are at least the length of the basal ring. Thus, these specimens appear to be intermediates between $N$. eobiapiculata and $N$. biapiculata. The decision to call these $N$. eobiapiculata was somewhat arbitrary and based upon the range of variation found in these samples.

\section{Navicula ponticulus (Ehrenberg)} (Plate 1, Figs. 5, 9)

Dictyocha ponticulus Ehrenberg, 1844, p. 258, 267; Bailey, 1845, pl. 4, fig. 21.

Naviculopsis ponticula (Ehrenberg), Bukry, 1978a, pl. 8, figs. 9, 10.

Remarks. This taxon has a relatively wide basal ring that narrows toward the long-axis end of the basal ring. The termination there is flattened. There is generally, but not always, a very short spine or vestigial swelling. There was considerable variation, especially in the axial-end of the basal ring. Some of the specimens counted here as Naviculopsis ponticulus might be counted as $N$. navicula or $N$. obtusarca by other workers (see Bukry, 1978a, 1982).

Two specimens of a strutted variant of this taxon were found in Section 113-689B-8H-2. Strutted naviculopsids are occasionally found in the geologic record (see Perch-Nielsen, 1976; Bukry, 1976c). Other silicoflagellates with a similar morphology are considered as Dictyocha (e.g., Ciesielski, 1975, pl. 7) on the basis of association. These variants are important because they give some insight into the relationship between Naviculopsis and Dictyocha, and to silicoflagellate variability in general. In the late Miocene, a similar group of naviculopsid morphologies appears to have evolved from Dictyocha brevis (see Bukry, 1978a; Locker and Martini, 1986).

\section{Naviculopsis trispinosa (Schulz)}

Dictyocha navicula trispinosa Schulz, 1928, p. 246, fig. 23a, b. Naviculopsis trispinosa (Schulz), Bukry, 1975c, p. 857, pl. 7, fig. 5-7.

Remarks. This species is very similar to $N$. biapiculata (see Bukry, $1975 \mathrm{c}$ ) and may be very closely related to it. Naviculopsis trispinosa, however, has a large apical spine, and is very distinctive because it usually lies on its side. The abundance of this taxon in samples in which five-sided Distephanus are also unusually abundant may indicate that this is an ecophenotype that occurs in high-stress environments. 


\section{Ebridians}

Genus ADONNADONNA Gombos, 1982 Adonnadonna primadonna Gombos

(Plate 7, Fig. 4)

Adonnadonna primadonna Gombos, 1982, p. 446.

Remarks. This is an extremely large and unusual ebridian (?) that is probably very closely related to, and in this study co-occurs with, Triskelion gorgon. The close relationship between these two taxa is indicated by the many similarities between them, including their enormous size, surface ornamentation, and co-occurrence in Section 113-689B-14H-5. Adonnadonna primadonna differs from Triskelion gorgon in having a circular inner ring from which skeletal elements radiate outward; the smaller ring is lacking in Triskelion gorgon.

\section{Genus AMMODOCHIUM Hovasse, 1932 \\ Ammodochium rectangulare (Schulz)}

(Pl. 7, Figs. 7, 8)

Ebria antigua var. rectangularis Schulz, 1928, p. 274, figs. 72 a-d. Ammodochium rectangulare (Schulz), Ling, 1971, p. 694.

Remarks. Ammonochium rectangulare is readily identified by its rectangular outline although, when positioned on its apical or antapical ring, the outline is triangular (Pl. 7, Fig. 8).

\section{Genus CRANIOPSIS Hovasse ex Frenguelli, 1940 \\ Craniopsis octo Hovasse ex Frenguelli, 1940}

(Plate 7, Fig. 5)

Craniopsis octo Hovasse ex Frenguelli, 1940, p. 95, figs. 31 a, b.

Remarks. This taxon was originally found by Hovasse (1932b) in the upper Eocene of New Zealand.

Genus EBRIOPSIS Hovasse, 1932
Ebriopsis antiqua antiqua (Schulz)

$$
\text { (Pl. 7, Fig. 6) }
$$

Ebriopsis antiqua Schulz, 1928 (in part), p. 273, 274, fig. 696. Ebriopsis antiqua antiqua (Schulz), Ling, 1977, p. 215, pl. 17, 18.

Remarks. Ebriopsis specimens with spines or pronounced surface crenulations were not found in this study.

\section{Genus PARATHRANIUM Hovasse, 1932 Parathranium tenuipes (Hovasse)}

Thranium tenuipes Hovasse, 1932a, p. 123, fig. 5. Parathranium tenuipes (Hovasse), Ling, 1972, p. 198-199.

\section{Genus TRISKELION Gombos, 1982 \\ Triskelion gorgon Gombos}

(Plate 7, Fig. 1-3)

Triskelion gorgon Gombos, 1982, p. 446-447.

Remarks. Gombos (1982) found that Triskelion gorgon appears to occur over a larger part of the middle Eocene than Adonnadonna primadonna.

\section{ACKNOWLEDGMENTS}

We are pleased to acknowledge the able assistance of Kristin Magnusen, who prepared the samples, Walter Thorner, for help and tutelage with computer graphics, and Dennis S. Cassidy, for generous help and use of the laboratories within the Antarctic Research Facility, FSU. Aboard ship, David Lazarus provided screen prints of some taxa that were quite helpful to the study. Support was provided by NSF Grant DPP 84-14268, JOI/USSAC funds, and an equipment grant from the AMOCO Foundation.

\section{REFERENCES}

Almgren, F. J., Jr., 1982. Minimal surface forms. Math. Intelligencer, 4:164-172.

Almgren, F. J., Jr., and Taylor, J. E., 1976. The geometry of soap films and soap bubbles. Sci. Am., 235:82-93.
Bachmann, A., and Ichikawa, W., 1962. The silicoflagellides in the Wakura Beds, Nanao City, Prefecture Ishikawa, Japan. Kanazawa Univ. Sci. Rept., 8:161-175.

Barker, P. F., Kennett, J. P., et al., 1988. Proc. ODP, Init. Repts., 113: College Station, TX (Ocean Drilling Program).

Barron, J. A., 1976. Revised Miocene and Pliocene diatom biostratigraphy of upper Newport Bay, Newport Beach, California. Mar. Micropaleontol., 1:27-63.

Barron, J. A., Bukry, D., and Poore, R. Z., 1984. Correlation of the middle Eocene Kellogg Shale of northern California. Micropaleontology, 30:138-170.

Boney, A. D., 1973. Observations on the silicoflagellate Dictyocha speculum Ehrenb. from the Firth of Clyde. J. Mar. Biol. Assoc. U. K., 53:263-268.

, 1976. Observations on the silicoflagellate Distephanus speculum Ehrenb.: double skeletons and mirror images. J. Mar. Biol. Assoc. U.K., 56:263-266.

1981. Distephanus speculum: Double skeletons with one aberrant partner. J. Mar. Biol. Assoc. U.K., 61:1027-1029.

Brasier, M. D., 1982. Architecture and evolution of the foraminiferal test-a theoretical approach. In Banner, F. T., and Lord, A. R. (Eds.), Aspects of Micropaleontology, London (Allen and Unwin), 1-41.

Bukry, D., 1973. Coccolith and silicoflagellate stratigraphy, Deep Sea Drilling Project Leg 18, Eastern North Pacific. In Kulm, L. D., von Huene, R., et al., Init. Repts. DSDP, 18: Washington (U.S. Govt. Printing Office), 817-831.

1975a. Coccolith and silicoflagellate stratigraphy near Antarctica, Deep Sea Drilling Project, Leg 28. In Hayes, D. E., Frakes, L. A., et al., Init. Repts. DSDP, 28: Washington (U.S. Govt. Printing Office), 709-723.

1975b. Coccolith and silicoflagellate stratigraphy, Northwestern Pacific Ocean, Deep Sea Drilling Project Leg 32. In Larson, R. L., Moberly, R., et al., Init. Repts. DSDP, 32: Washington (U.S. Govt. Printing Office), 677-701.

1975c. Silicoflagellate and coccolith stratigraphy, Deep Sea Drilling Project Leg 29. In Kennett, J. P., Houtz, R. E., et al., Init. Repts. DSDP, 29: Washington (U.S. Govt. Printing Office), 845872.

1976a. Cenozoic silicoflagellate and coccolith stratigraphy, South Atlantic Ocean, Deep Sea Drilling Project Leg 36. In Hollister, C. D., Craddock, C., et al., Init. Repts. DSDP, 35: Washington (U.S. Govt. Printing Office), 885-917.

1976b. Comments on some coccoliths and silicoflagellates from Deep Sea Drilling Project Leg 35. In Hollister, C. D., Craddock, C., et al., Init. Repts. DSDP, 35: Washington (U.S. Govt. Printing Office), 693-699.

1976c. Silicoflagellate and coccolith stratigraphy, NorwegianGreenland Sea, Deep Sea Drilling Project Leg 38. In Talwani, M., Udintsev, G., et al., Init. Repts. DSDP, 38: Washington (U.S. Govt. Printing Office), 843-855.

1976d. Silicoflagellate and coccolith stratigraphy, southeastern Pacific Oceans, Deep Sea Drilling Project Leg 34. In Yeats, R. S., Hart, S. R., et al., Init. Repts. DSDP, 34: Washington (U.S. Govt. Printing Office), 715-735.

1977. Coccolith and silicoflagellate stratigraphy, South Atlantic Ocean, Deep Sea Drilling Project Leg 39. In Perch-Nielsen, K., Supko, P. R., et al., Init. Repts. DSDP, 39: Washington (U.S. Govt. Printing Office), 825-840.

1978a. Cenozoic coccolith, silicoflagellate, and diatom stratigraphy, Deep Sea Drilling Project Leg 44. In Benson, W. E., Sheridan, R. E., et al., Init. Repts. DSDP, 44: Washington (U.S. Govt. Printing Office), 807-863.

1978b. Cenozoic silicoflagellate and coccolith stratigraphy, northwestern Atlantic Ocean, Deep Sea Drilling Leg 43. In Benson, W. E., Sheridan, R. E., et al., Init. Repts. DSDP, 44: Washington (U.S. Govt. Printing Office), 775-805.

1978c. Cenozoic silicoflagellate and coccolith stratigraphy, southeastern Atlantic Ocean, Deep Sea Drilling Project Leg 40. In Bolli, H. M., Ryan, W.B.F., et al., Init. Repts. DSDP, 40: Washington (U.S. Govt. Printing Office), 635-649.

1979. Coccolith and silicoflagellate stratigraphy, northern Mid-Atlantic ridge and Reykjanes Ridge, Deep Sea Drilling Project Leg 49. In Luyendyk, B. P., Cann, J. R., et. al., Init. Repts. DSDP, 49: Washington (U.S. Govt. Printing Office), 551-581. 
1981. Synthesis of silicoflagellate stratigraphy for Maastrichtian to Quaternary marine sediment. Soc. Econ. Paleontol. Mineral. Spec. Publ., 32:433-444.

1982. Cenozoic silicoflagellates from offshore Guatemala, Deep Sea Drilling Project Site 495. In Aubouin, J., von Huene, R., et al., Init. Repts. DSDP, 67: Washington (U.S. Govt. Printing Office), $425-445$.

1983. Upper Cenozoic silicoflagellates from offshore Ecuador, Deep Sea Drilling Project Site 504. In Cann, J. R., Langseth, M. G., et al., Init. Repts. DSDP, 69: Washington (U.S. Govt. Printing Office), 321-342.

1985. Tropical Pacific silicoflagellate zonation and paleotemperature trends of the Late Cenozoic. In Mayer, L., Theyer, F., et al., Init. Repts. DSDP, 85: Washington (U.S. Govt. Printing Office), 477-497.

, 1987. Eocene siliceous and calcareous phytoplankton, Deep Sea Drilling Project Leg 95. In Poag, C. W., Watts, A. B., et al., Init. Repts. DSDP, 95: Washington (U.S. Govt. Printing Office), 395-415.

Bukry, D., and Foster, J. H., 1973. Silicoflagellate and diatom stratigraphy, Leg 16, Deep Sea Drilling Project. In Van Andel, T. H., Heath, G. R., et al., Init. Repts. DSDP, 16: Washington (U.S. Govt. Printing Office), 815-871.

Burger, W. C., 1975. The species concept in Quercus. Taxon, 24:45-50.

Busen, K. E., and Wise, S. W., Jr., 1977. Silicoflagellate stratigraphy, Deep Sea Drilling Project, Leg 36. In Barker, P., Dalziel, I.W.D., et al., Init. Repts. DSDP, 36: Washington (U.S. Govt. Printing Office), 697-743.

Ciesielski, P. F., 1974. Neogene and Oligocene silicoflagellate biostratigraphic zonation for the southern ocean. U.S. Antarct. J., 9:271-272. 1975. Biostratigraphy and paleoecology of Neogene and Oligocene silicoflagellates from cores recovered during Antarctic Leg 28, Deep Sea Drilling Project. In Hayes, D. E., Frakes, L. A., et al., Init. Repts. DSDP, 28: Washington (U.S. Govt. Printing Office), 625-691.

Ciesielski, P. F., Hasson, P., and Turner, J. W., Jr., 1989. The stratigraphy of silicoflagellates from the Norwegian Sea, ODP Leg 104. In Eldholm, O., Thiede, J., et al., 1988. Proc. ODP, Sci. Results, 104: College Station, TX (Ocean Drilling Program), 497-526.

Ciesielski, P. F., and Weaver, F. M., 1974. Early Pliocene temperature changes in the Antarctic seas. Geology, 2:511-516.

1983. Neogene and Quaternary paleoenvironmental history of Deep Sea Drilling Project Leg 71 sediments, Southwest Atlantic Ocean. In Ludwig, W. J., Krasheninnikov, V. A., et al., Init. Repts. $D S D P, 71$, Pt. 2: Washington (U.S. Govt. Printing Office), 461-477.

Conway, K., and Trainor, F. R., 1972. Scenedesmus morphology and flotation. J. Phycol., 8:138-143.

Deflandre, G., 1950. Contribution a l'etude des silicoflagellides actuels et fossiles. Microscopie, 2:72-108, 117-142, and 191-210.

Dumitrič, P., 1973. Paleocene, Late-Oligocene and post-Oligocene silicoflagellates in southwestern Pacific sediment cored on DSDP Leg 21. In Burns, R. E., Andrews, J. E., et al., Init. Repts. DSDP, 21 : Washington (U.S. Govt. Printing Office), 837-883.

Dzinoridze, R. N., Jouse, A. P., Koroleva-Golikova, G. S., Kozlova, G. E., Nagaeva, G. S., Petrushevskaya, M. G., and Strelnikova, N. I., 1978. Diatom and radiolarian Cenozoic stratigraphy, Norwegian Basin; DSDP Leg 38. In Talwani, M., Udintsev, G., et al., Init. Repts. DSDP, Suppl. to Vols. 38, 39, 40, 41: Washington (U.S. Govt. Printing Office), 289-427.

Ehrenberg, C. G., 1839. Über die Bildung der Kreidefelsen und des Kreidemergels durch unsichtbare Organismen. Abh. Preuss. Akad. Wiss. Berlin, Jahrg., 59-148.

1841. 274 Blätter von ihm selbst ausgeführten Zeichnungen von ebenso vielen Arten. Ber. Preuss. Akad. Wiss. Berlin, Jahrg., 197-219.

1844. Mitteilung über zwei neue Lager von Gebirgsmassen aus Infusorien als Meeres-Absatz in Nord-Amerika und eine Vergleichung derselben mit den organischen Kreide-Gebilden in Europa und Afrika. Ber. Preuss. Akad. Wiss. Berlin, Jahrg., 57-97. 1854. Mikrogeologie: Leipzig (Leopold Voss).
Frenguelli, J., 1935. Variaciones de Dictyocha fibula en el Golfo de San Matías. Ann. Mus. Argentino Cienc. Nat., 38:263-381.

1938. Variaciones des "Dictyocha speculum" Ehr. en el Golfo de San Jorge (Patagonia. La Plata Univ. Nac., Inst. Mus. La Plata, 3:117-123.

1940. Consideraciones sobre los sílicoflagelados fósiles. Rev Mus. La Plata, Ser. 2, 2:37-112.

Gemeinhardt, K., 1930. Silicoflagellatae. In Rabenhorst, L., Kryptogamen-Flora von Deutschland, Österreich und der Schweiz. Leipzig Akad. Verilagsgesellschaft, 10:1-87.

Glezer, Z. I., 1966. Silicoflagellatophycaea. In Gollerbakh, M. M. (Ed.) Cryptogamic Plants of the U.S.S.R. (Vol. 7). V. A. Komarova Bot. Inst., 1-363. (Transl, from Russian by Israel Program for Scientific Translations Ltd., Jerusalem, 1970.)

Gombos, A. M., Jr., 1982. Three new and unusual genera of ebridians from the southwest Atlantic Ocean. J. Paleontol., 56:444-448.

Gran, H. H., and Braarud, T., 1935. A quantitative study of the phytoplankton in the Bay of Fundy and the Gulf of Maine (including observations of the hydrogeology, chemistry and turbidity). J. Biol. Board Can. 1:279-467.

Haeckel, E., 1887. Report on the Scientific Results of the Voyage of the H. M. S. Challenger. Zoology, 18:1-1803.

Hajos, M., 1975. Late Cretaceous archaeomonads, diatomaceae, and silicoflagellata from the South Pacific Ocean, Deep Sea Drilling Project, Leg 29, Site 275. In Kennett, J. P., Houtz, R. E., et al., Init. Repts. DSDP, 29: Washington (U.S. Govt. Printing Office), 9131010.

1976. Upper Eocene and lower Oligocene Diatomaceae, Archaeomonadaceae, and Silicoflagellatae in southwestern Pacific sediments, DSDP Leg 29. In Hollister, C. D., Craddock, C., et al., Init. Repts. DSDP, 35: Washington (U.S. Govt. Printing Office), 817883.

Hanna, G. D., 1928. Silicoflagellata from the Cretaceous of California. J. Paleontol., 1:259-263.

1931. Diatoms and silicoflagellates of the Kreyenhagen shale. Calif. Div. Mines Geol. State Mineral. Rep., 27:187-201.

Haq, B. U., and Riley, A., 1976. Antarctic silicoflagellates from the southeast Pacific, Deep Sea Drilling Project Leg 35. In Hollister, C. D., Craddock, C., et al., Init. Repts. DSDP, 35: Washington (U.S. Govt. Printing Office), 673-692.

Hovasse, R., 1932a. Note préliminaire sur les Ébriacées. Soc. Zool. Pr. Bull., 57:118-131. 57:457-476.

1932b. Troisième note sur les Ébriacées. Soc. Zool. Pr. Bull., 1932c. Contribution a l'étude des silicoflagellés. Multiplication, variabilité, hérédite, affinités. Bull. Biol. Pr. Belgique, 66:447501.

Ichikawa, W., Fuji, N., and Bachmann, S., 1964. Microfossils found from Noto, Japan. Sci. Repts. Kanazawa Univ., 9:25-118.

Isenberg, C., 1976. The soap film: an analog computer. Am. Sci., 64: 514-518.

Kennett, J. P., 1978. The development of planktonic biogeography in the Southern Ocean during the Cenozoic. Mar. Micropaleontol., 3: 301-345.

Kitchell, J., 1983. Biotic interactions and siliceous marine phytoplankton: An ecological and evolutionary perspective. In Tevesz, M.J.S., and McCall, P. L., (Eds.), Biotic Interactions in Recent and Fossil Benthic Communities, New York (Plenum Press), 285-329.

Lemmermann, E., 1901. Silicoflagellatae. Deutsche Bot. Ges. Ber., 19: 247-271.

Ling, H. Y., 1972. Upper Cretaceous and Cenozoic silicoflagellates and ebridians. Bull. Am. Paleontol., 62:135-229.

1977. Late Cenozoic silicoflagellates and ebridians from the Eastern North Pacific region. Proc. First Int. Congr. Pacific Neogene Stratigraphy Tokyo, 1976: Tokyo (Kaiyo Shuppan Co.), 205233.

Ling, H. Y., and McPherson, L. M., 1974. Study on the ebridian genus Parathranium Hovasse. Rev. Esp. Micropaleontol., 6:191-200.

Locker, S., and Martini, E., 1986. Silicoflagellates and some sponge spicules from the southwest Pacific, Deep Sea Drilling Project, Leg 
90. In Kennett, J. P., von der Borch, C. C., et al., Init. Repts. DSDP, 90: Washington (U.S. Govt. Printing Office), 887-924. 1987. Silicoflagellaten aus einigen russischen Palaogen-Vorkommen. Senkenbergiana lethaea 68:21-67.

Loeblich, A. R., III, Loeblich, L. A., Tappan, H., and Loeblich, A. R., Jr., 1968. Annotated Index of Fossil and Recent Silicoflagellates and Ebridians with Descriptions and Illustrations of Validly Proposed Taxa. Geol. Soc. Am. Mem., 106.

Mandra, Y. T., 1968. Silicoflagellates from the Cretaceous, Eocene, and Miocene of California, U. S. A. Proc. Calif. Acad. Sci., 36:231-277.

Mandra, Y. T., and Mandra, H., 1972. Paleoecology and taxonomy of silicoflagellates from an upper Miocene diatomite near San Felipe, Baja California, Mexico. Occasional Papers, Calif. Acad. Sci., 99: $1-35$.

Martini, E., 1971. Neogene silicoflagellates from the equatorial Pacific. In Winterer, E. L., Riedel, W. R., et al., Init. Repts. DSDP, 7, Pt. 2: Washington (U.S. Govt. Printing Office), 1695-1708.

, 1977. Systematics, distribution and stratigraphic application of silicoflagellates. In Ramsay, A. T. S. (Ed.), Oceanic Micropaleontology. New York (Academic Press), 1327-1343.

Martini, E., and Muller, C., 1976. Eocene to Pleistocene silicoflagellates from the Norwegian-Greenland Sea (DSDP Leg 38). In Talwani, M., and Udintsev, G., et al., Init. Repts. DSDP, 38: Washington (U.S. Govt. Printing Office), 857-895.

McCartney, K., 1987. Silicoflagellates, ebridians and archaeomonads. In Fossil Procrayotes and Protists: In Broadhead, T. W. (Ed.), Notes for a Short Course, Paleontol. Soc. and Cushman Found., Univ. Tennessee, Knoxville, 146-168.

McCartney, K., and Loper, D. E., 1989. Optimized skeletal morphologies of silicoflagellate genera Dictyocha and Distephanus. Paleobiology, 15:283-298.

McCartney, K., and Wise, S. W., Jr., 1987. Silicoflagellates and ebridians from Deep Sea Drilling Project Leg 93, Site 604 and 605 (New Jersey Transect). In van Hinte, J. E., Wise, S. W., Jr., et al., Init. Repts. DSDP, 93: Washington (U.S. Govt. Printing Office), 801814.

McGhee, G. R., Jr., 1980. Shell form in the convex articulate Brachiopoda: a geometrical analysis. Paleobiology, 6:57-76.

Mercer, J. H., 1978. Glacial development and temperature trends in the Antarctic and in South America. In van Zinderen Bakker, E. M. (Ed.), Antarctic Glacial History and World Paleoenvironments: Rotterdam (A. A. Balkema), 73-94.

Perch-Nielsen, K., 1975a. Late Cretaceous to Pleistocene archaeomonads, ebridians, endoskeletal dinoflagellates, and other siliceous microfossils from the subantarctic southwest Pacific, DSDP Leg 29. In Kennett, J. P., Houtz, R. E., et al., Init. Repts. DSDP, 29: Washington (U.S. Govt. Printing Office), 873-907.

1975b. Late Cretaceous to Pleistocene silicoflagellates from the southern southwest Pacific, DSDP Leg 29. In Kennett, J. P., Houtz, R. E., et al., Init. Repts. DSDP, 29: Washington (U.S. Govt. Printing Office), 677-721.

1975c. New silicoflagellates and a silicoflagellate zonation in north European Palaeocene and Eocene diatomites. Bull. Geol. Soc. Den., 25:27-40.

1976. Eocene to Pliocene archaeomonads, ebridians, and endoskeletal dinoflagellates from the Norwegian Sea, DSDP Leg 38. In Talwani, M., Udintsev, G., et al., Init. Repts. DSDP, Suppl. to Vols. 38, 39, 40, and 41: Washington (U.S. Govt. Printing Office), 147-175.

, 1985. Silicoflagellates. In Bolli, H. M., Saunders, J. B., and Perch-Nielsen, K. (Eds.), Plankton Stratigraphy: Cambridge (Cambridge Univ. Press), 811-846.

Poelchau, H. S., 1974. Holocene silicoflagellates of the North Pacific: their distribution and use for paleotemperature determination. [Ph.D. dissert.] Univ. of California, San Diego.

1976. Distribution of Holocene silicoflagellates in North Pacific sediments. Micropaleontology, 22:164-193.

Raup, D. M., 1968. Theoretical morphology of echinoid growth. J. Paleontol., Suppl., 42:50-63.
Sargeant, W. A. S., Lacalli, T., and Gaines, G., 1987. The cysts and skeletal elements of dinoflagellates: speculations on the ecological causes for their morphology and development. Micropaleontology, 33:1-36.

Schrader, H. J., 1972. Kieselsaure-Skelette in Sedimenten des iberomarokkanischen Kontinentalrandes und angrenzender Tiefsee-Ebenen. "Meteor"-Forsch. Ergebuisse, 8:10-36.

Schrader, H., Pisias, N., and Cheng, G., 1986. Seasonal variation of silicoflagellates in phytoplankton and varved sediments in the Gulf of California. Mar. Micropaleontol., 10:207-233.

Schulz, P., 1928. Beiträge zur Kenntnis fossiler und rezenter Silicoflagellaten. Bot. Arch., 21:225-292.

Shaw, C. A., and Ciesielski, P. F., 1983. Silicoflagellate biostratigraphy of middle Eocene to Holocene subantarctic sediments recovered by Deep Sea Drilling Project Leg 71. In Ludwig, W. J., Krasheninnikov, V. A., et al., Init. Repts. DSDP, 71: Washington (U.S. Govt. Printing Office), 687-737.

Shipboard Scientific Party, 1988a. Site 689. In Barker, P. F., Kennett, J. P., et al., Proc. ODP, Init. Repts., 113: College Station, TX (Ocean Drilling Program), 89-181.

, 1988b. Site 693. In Barker, P. F., Kennett, J. P., et al., Proc. ODP, Init. Repts., 113: College Station, TX (Ocean Drilling Program), 329-447.

1988c. Site 696. In Barker, P. F., Kennett, J. P., et al., Proc. ODP, Init. Repts., 113: College Station, TX (Ocean Drilling Program), 607-704.

Shitanaka, M., 1983. Silicoflagellate remains in the sediments of Lake Hiruga, Fukui, Japan. Bull. Mizunami Fossil Mus., 10:171-180.

Smith, C. S., 1954. The shape of things. Sci. Am., 190:58-64.

Tappan, H., 1980. The Paleobiology of Plant Protists. San Francisco (W. H. Freeman).

Thompson, D'A. W., 1942. On growth and form. Cambridge (Cambridge Univ. Press) (2nd ed.).

Tsumura, K., 1963. A systematic study of silicoflagellateae. Yokohama Mun. Univ. J. C-45, 146:184.

Van der Spoel, S., Hallegaeff, G. M., and Van Soest, R.W.M., 1973. Notes on variation of diatoms and silicoflagellates in the South Atlantic Ocean. Neth. J. Sea Res., 6:518-541.

Van Valen, L., 1976. Ecological species, multispecies, and oaks. Taxon, $25: 233-239$.

Van Valkenburg, S. D., 1970. The ultrastructure of the silicoflagellate Dictyocha fibula Ehrenberg [Ph.D. dissert.]. Univ. of Washington, Seattle.

1980. Silicoflagellates. In Cox, E. R. (Ed.), Phytoflagellates, Holland (Elsevier North Holland, Inc.), 335-350.

Van Valkenburg, S. D., and Norris, R. E., 1970. The growth and morphology of the silicoflagellate Dictyocha fibula Ehrenberg in culture. J. Phycol., 6:48-54.

Walsby, A. E., and Xypolyta, A., 1977. The form resistance of chitin fibres attached to the cells of Thalassiostra fluviatilis Hustedt. Phycol. J., 12:215-223.

Wise, S. W., Jr., 1981. Deep sea drilling in the Antarctic: focus on late Miocene glaciation and applications of smear-slide biostratigraphy. In Warme, J. E., Douglas, R. G., and Winterer, E. L. (Eds.), The Deep Sea Drilling Project: A Decade of Progress, Soc. Econ. Pal. Min. Spec. Publ., 32:471-487.

Wise, S. W., Jr., Gombos, A. M., and Muza, J. P., 1985. Cenozoic evolution of polar water masses, southwest Atlantic Ocean. In Hsu, K. J., and Weissert, H. J. (Eds.), South Atlantic Paleoceanography: Cambridge (Cambridge Univ. Press), 283-324.

Yanagisawa, T., 1943. Klishitsu-benmochu nitsuite (Silicoflagellatae). Umi to Sora, 23:11-29.

Date of initial receipt: 27 February 1988

Date of acceptance: 19 September 1989

Ms 113B-142 


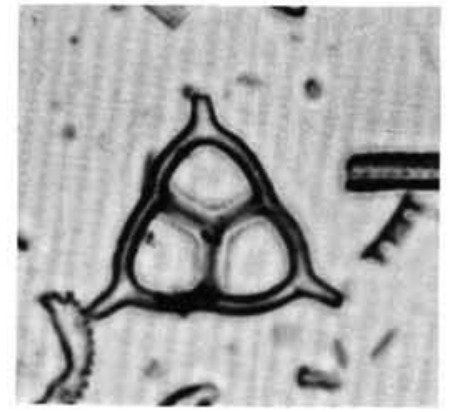

1

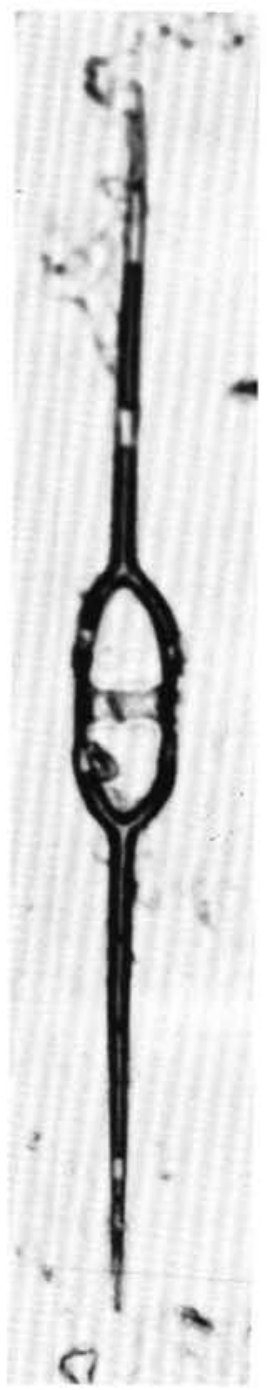

6

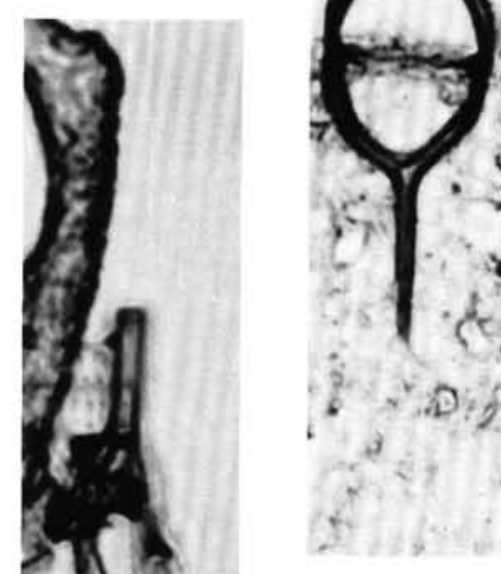

2

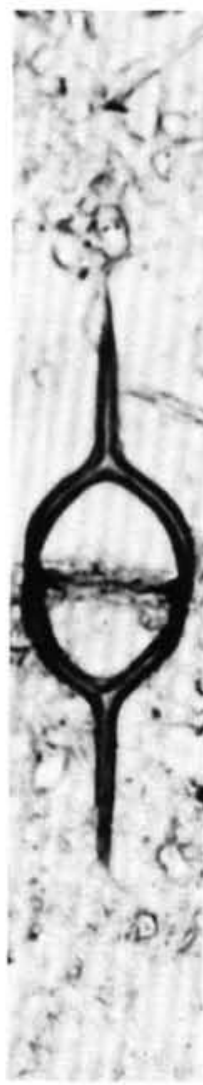

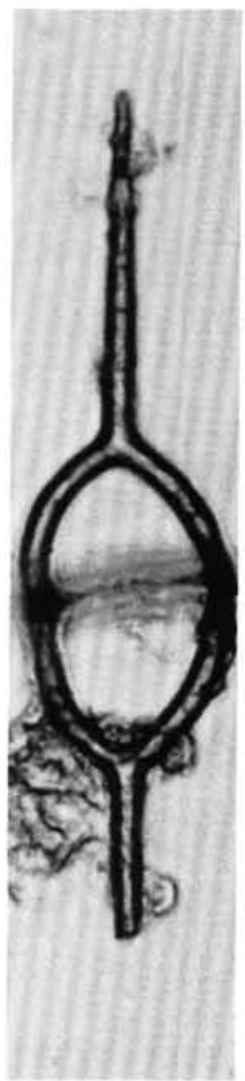

3

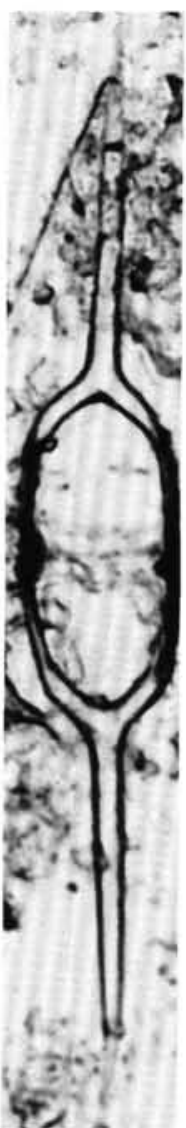

4

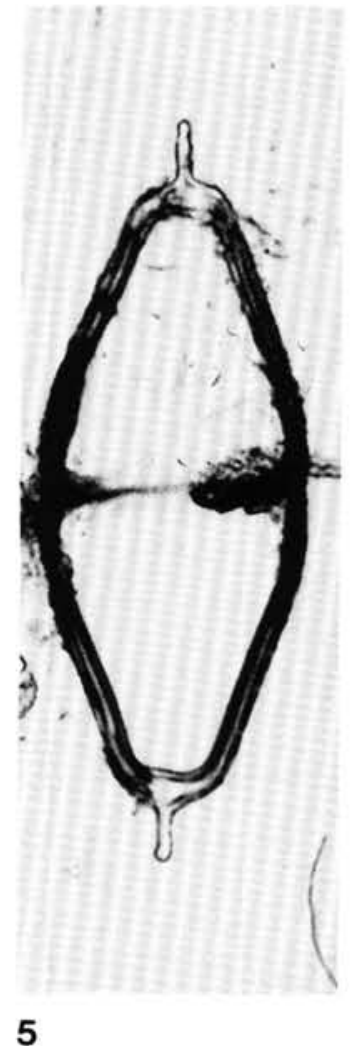

5

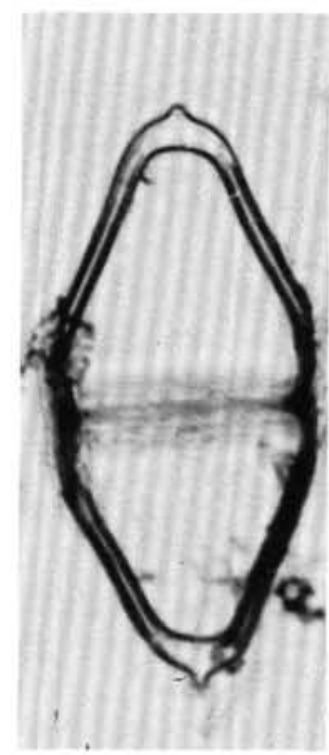

9

Plate 1. Corbisema, Dictyocha, and Naviculopsis from Hole 689B, ODP Leg 113, 740×. 1. Corbisema triacantha (Ehrenberg), Sample 113689B-7H-5, 29-31 cm. 2-4. Naviculopsis eobiapiculata Bukry, (2) Sample 113-689B-12H-2, 29-31 cm; (3) Sample 113-689B-9H-2, 29-31 cm; (4) Sample 113-689B-11H-2, 32-34 cm. 5. Naviculopsis ponticulus (Ehrenberg), Sample 113-689B-8H-2, 30-32 cm. 6-7. Naviculopsis constricta (Schulz), (6) Sample 113-689B-13H-5, 28-30 cm; (7) Sample 113-689B-14H-5, 30-32 cm. 8. Dictyocha pentagona (Schulz), Sample 113-689B-2H-2, 33-35 cm. 9. Naviculopsis ponticulus (Ehrenberg), Sample 113-689B-8H-2, 30-32 cm. 


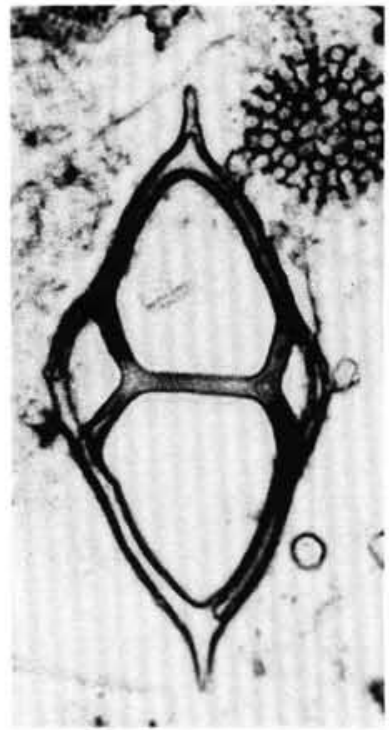

1
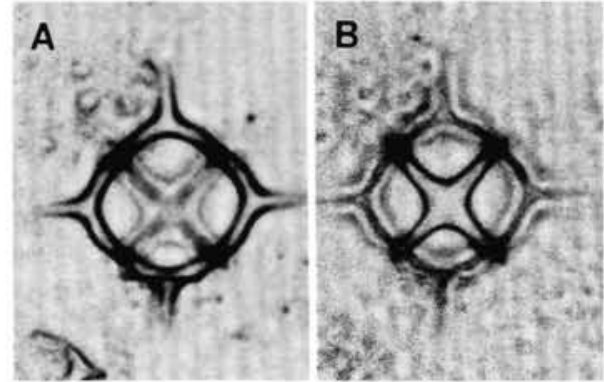

5

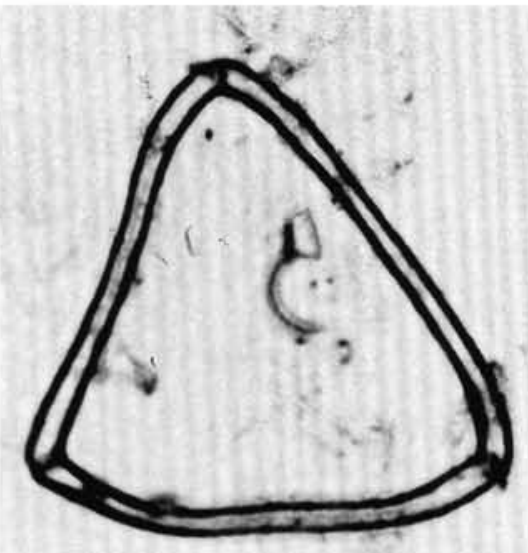

8

2

3
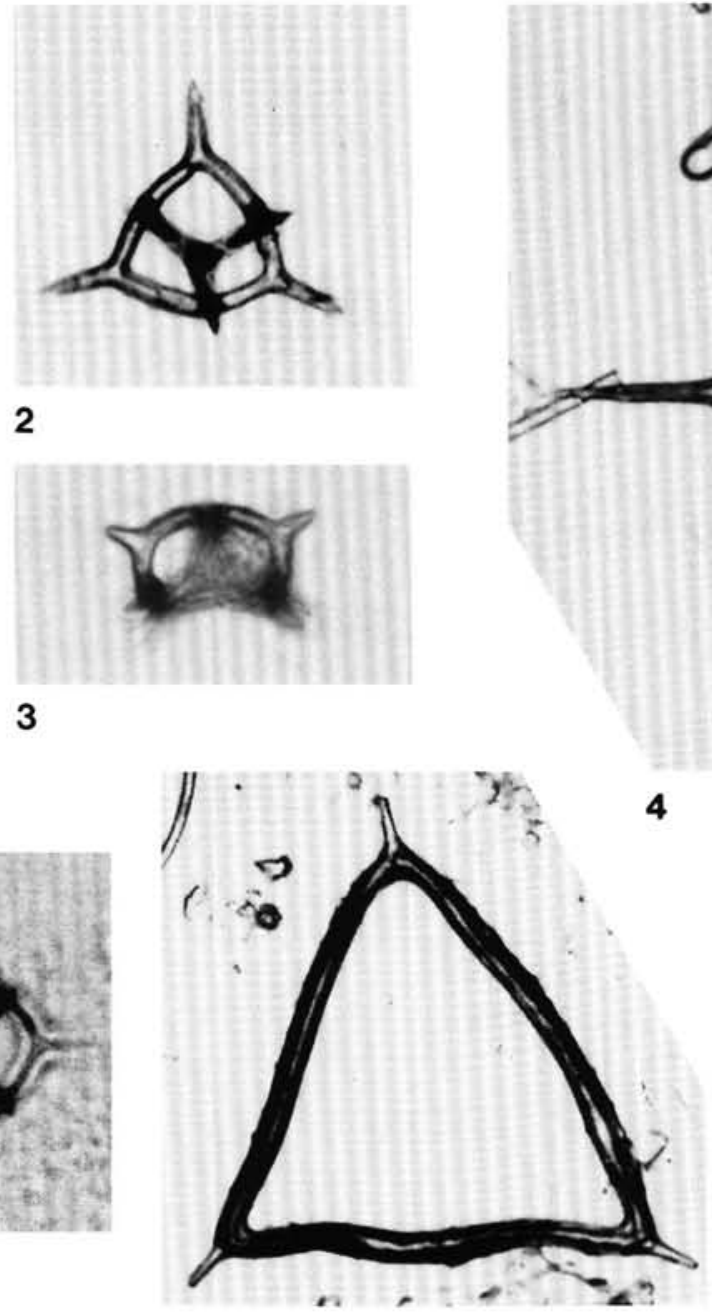

6

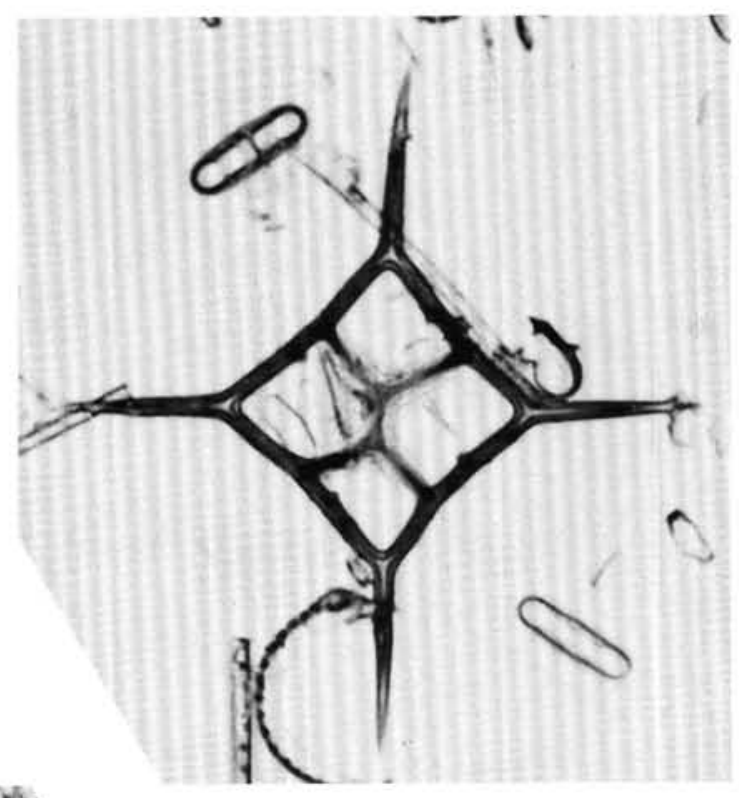

4
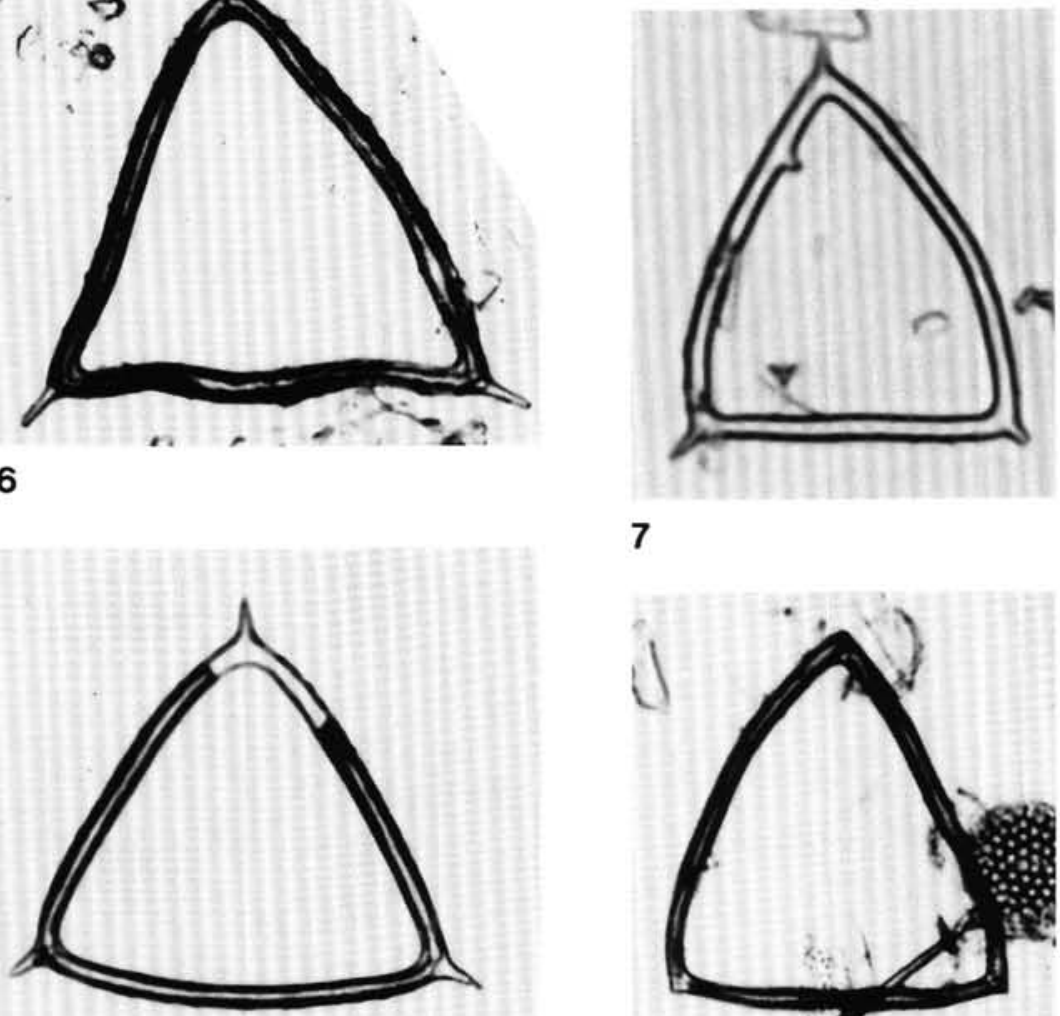

9
7

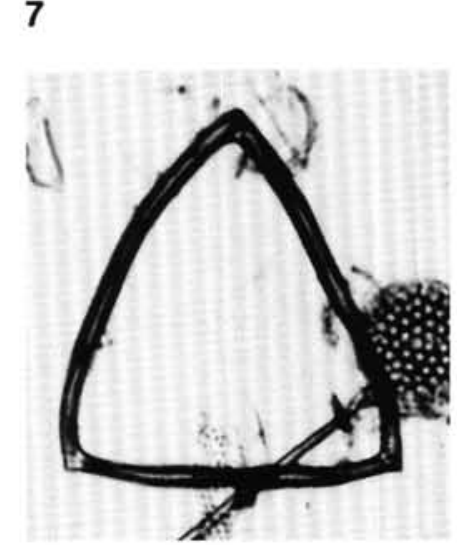

10

Plate 2. Dictyocha and Bachmannocena from ODP Leg 113, 740×. 1. Dictyocha aspera, Sample 113-690B-6H-2, 28-30 cm. 2. Dictyocha spinosa (Deflandre), Sample 113-689B-9H-2, 29-31 cm. 3. Dictyocha frenguellii Deflandre, lateral view, Sample 113-689B-9H-5, 29-31 cm. 4. Dictyocha calida Poelchau, abapical view, Sample 113-689B-5H-5, 29-31 cm. 5. Dictyocha deflandrei Frenguelli, Sample 113-689B-10H-5, 30-32 cm. 6-10. Bachmannocena apiculata Schulz, showing range of variation, (6) Sample 113-689B-11 H-2, 32-34 cm; (7) Sample 113-689B-8H-5, 30-32 cm; (8) Sample 113-689B-8H-2, 30-32 cm; (9) Sample 113-689B-8H-5, 30-32 cm; (10) Sample 113-689B-8H-2, 30-32 cm. 


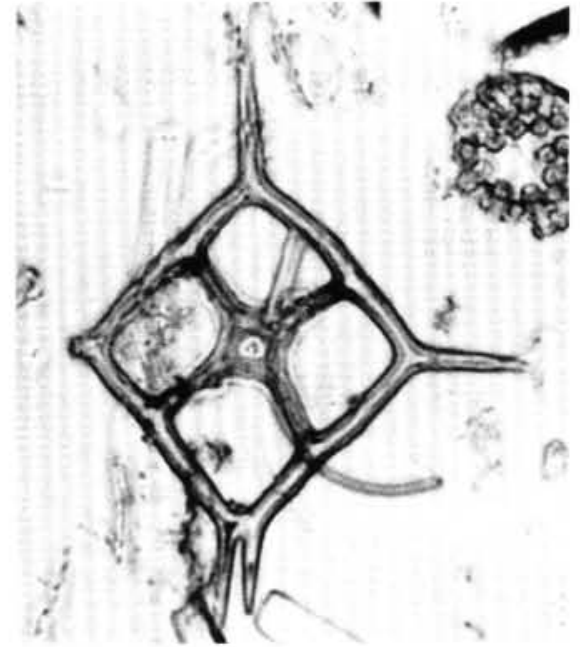

1

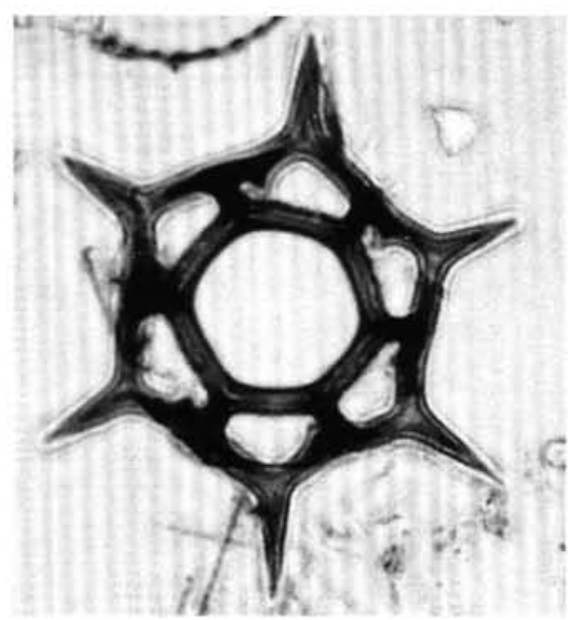

4
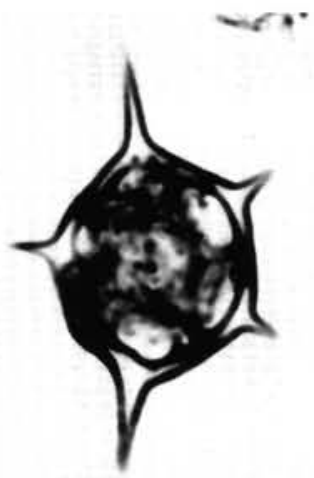

7

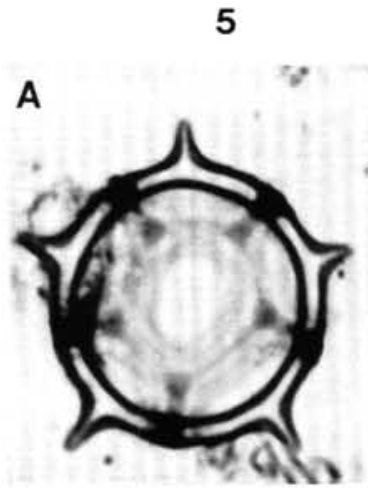

8

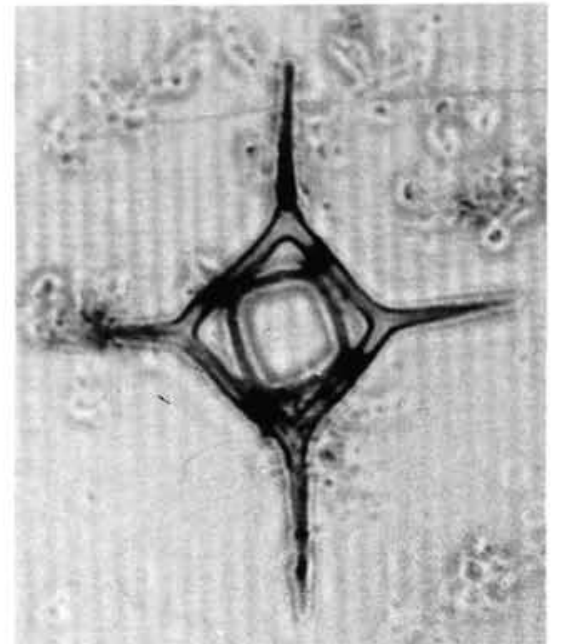

2

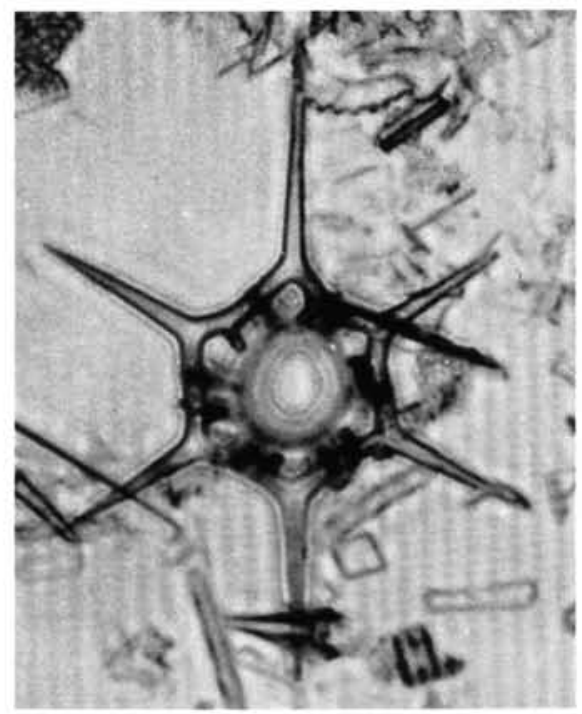

5

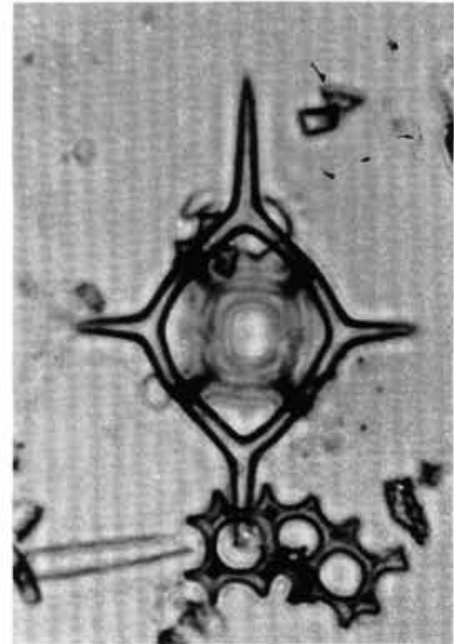

3

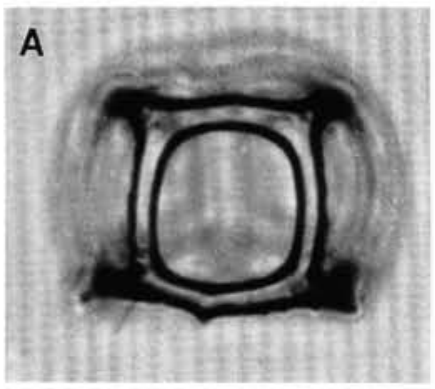

B

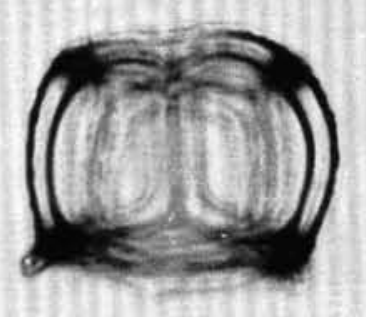

C

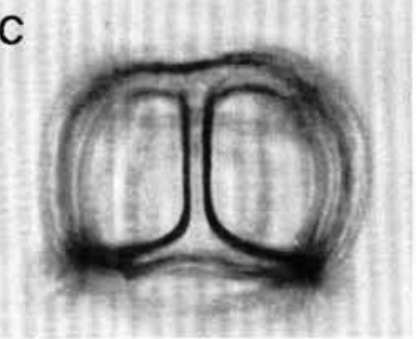

6

Plate 3. Distephanus from Hole 689B, Leg 113, 740× 1-2. Distephanus crux (Ehrenberg), (1) Sample 113-689B-6H-5, 29-31 cm; (2) Sample 113689B-13H-5, 28-30 cm. 3. Distephanus longispinus, Sample 113-689B-8H-5, 30-32 cm. 4. Distephanus boliviensis (Frenguelli), Sample 113-689B3H-5, 29-31 cm. 5. Distephanus speculum speculum (Ehrenberg), Sample 113-689B-4H-5, 30-32 cm. 6A-C. Distephanus crux/speculum, Sample 113-689B-9H-2, 29-31 cm. 7, 8A-B. Distephanus speculum pentagonus Lemmermann, Sample 113-689B-6H-5, 29-31 cm. 

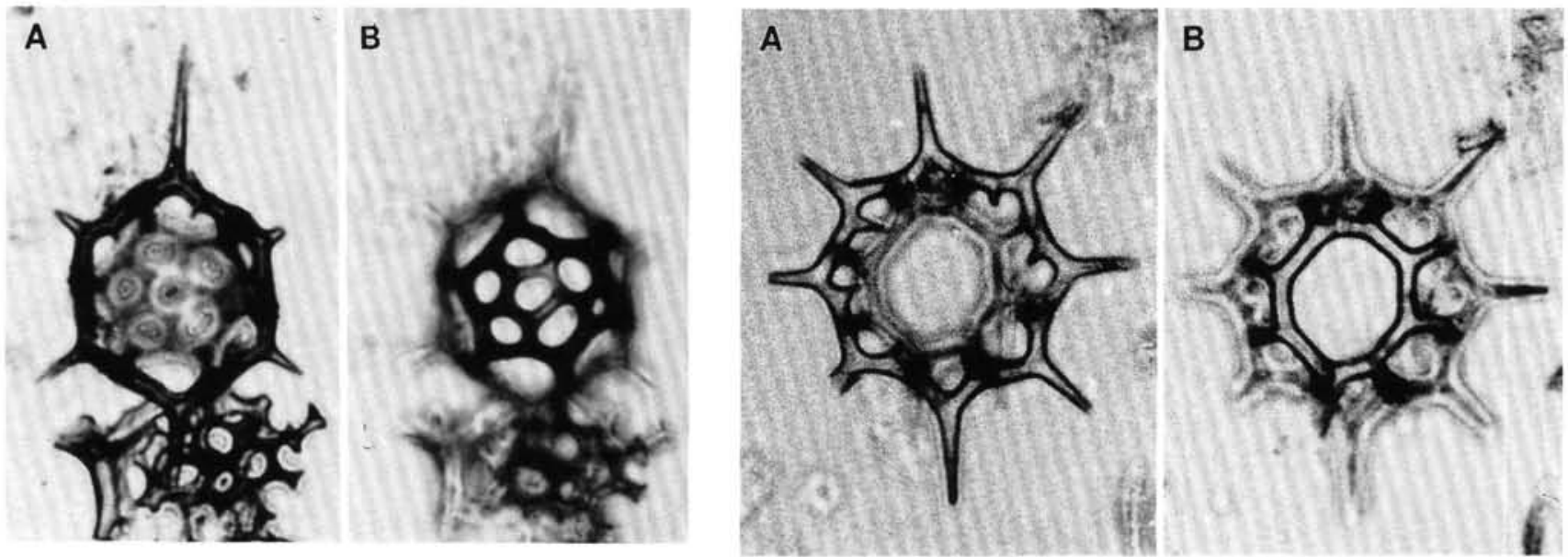

1

2
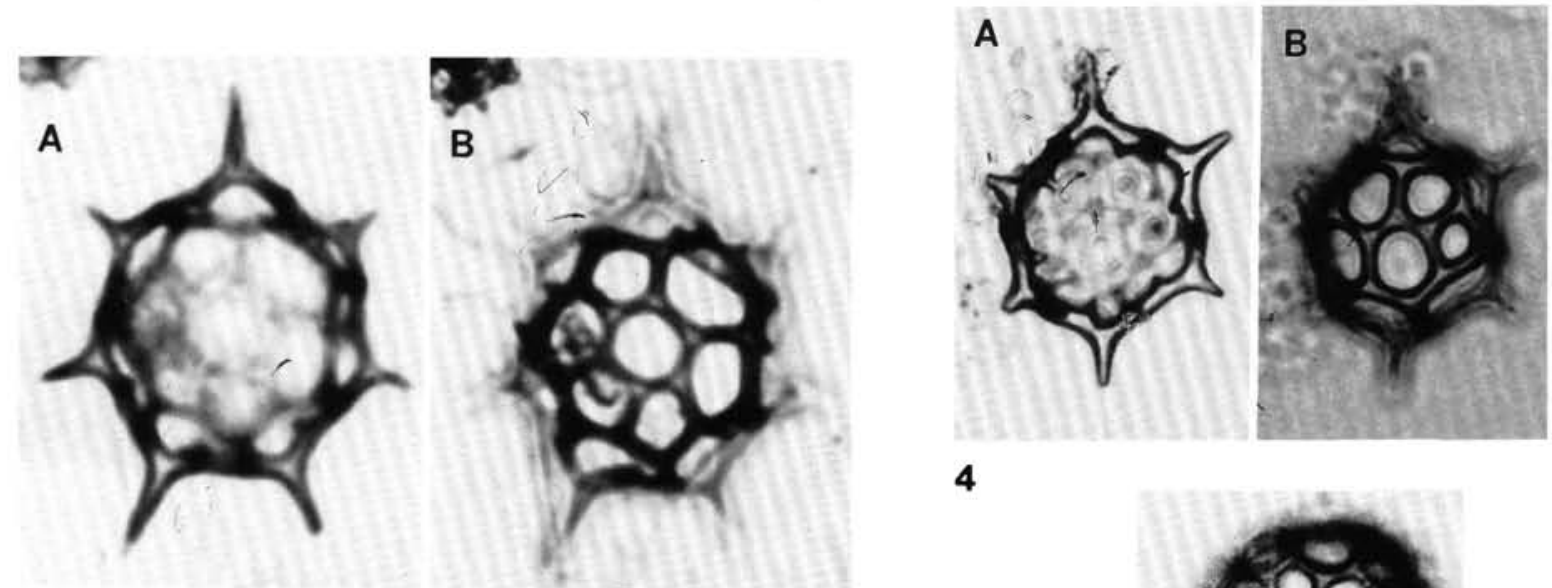

\section{4}

3

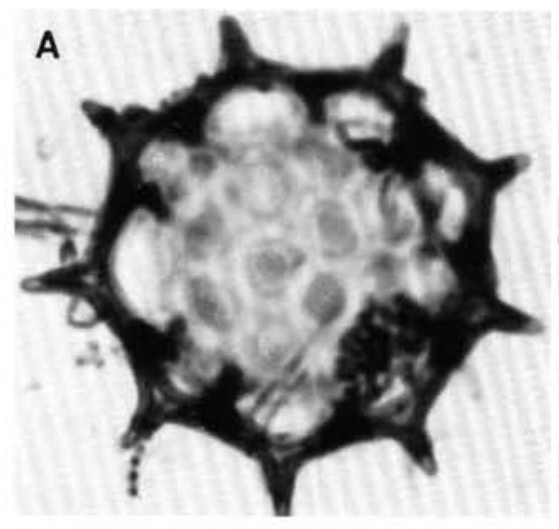

5

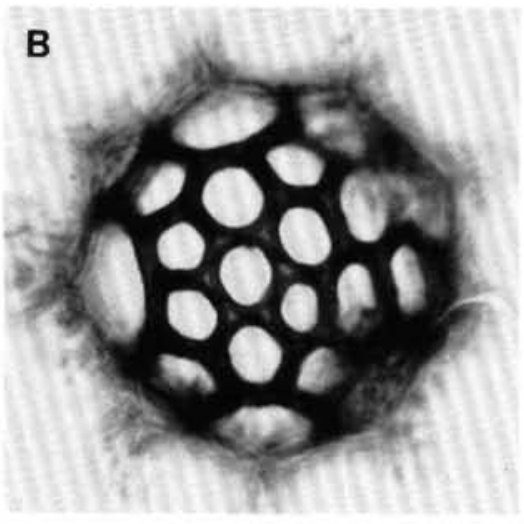

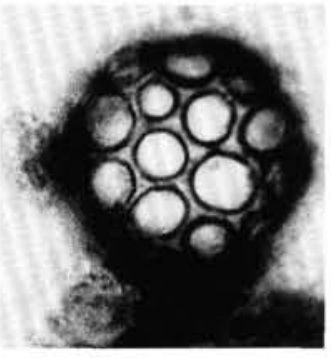

6

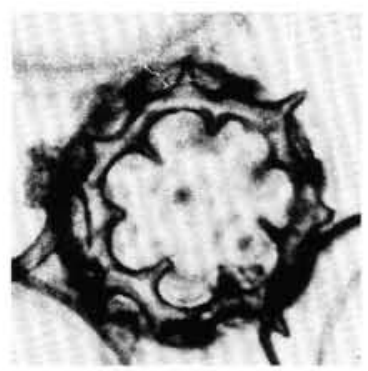

7

Plate 4. Multiwindowed and many-sided silicoflagellates from ODP Leg 113, 740 $\times$. 1A-B. Distephanus speculum hemisphaericus (high and low focus), Sample 113-689B-8H-5, 30-32 cm. 2A-B. Distephanus speculum speculum (eight-sided, high and low focus), Sample 113-690B-2H-1, 30-32 $\mathrm{cm}$. 3-7. Distephanus speculum hemisphaericus, (3A-B) (high and low focus), Sample 113-689B-7H-5, 29-31 cm. (4A-B) (high and low focus), 113-689B-10H-5, 29-31 cm. (5A-B) (high and low focus), Sample 113-689B-7H-5, 29-31 cm. (6, 7) (apical and abapical views, different specimens), Sample 113-689B-7H-5, 29-31 cm. 


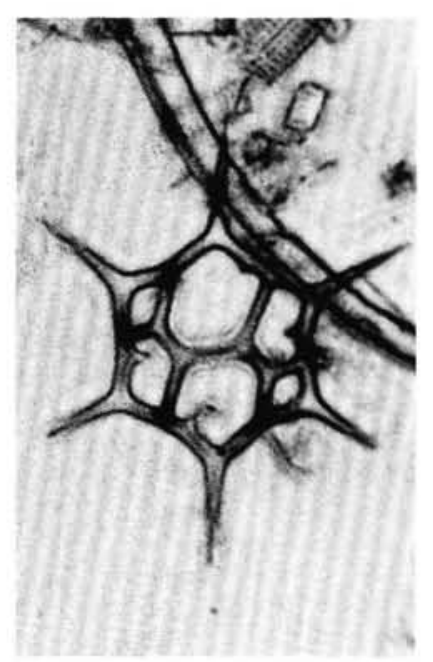

1

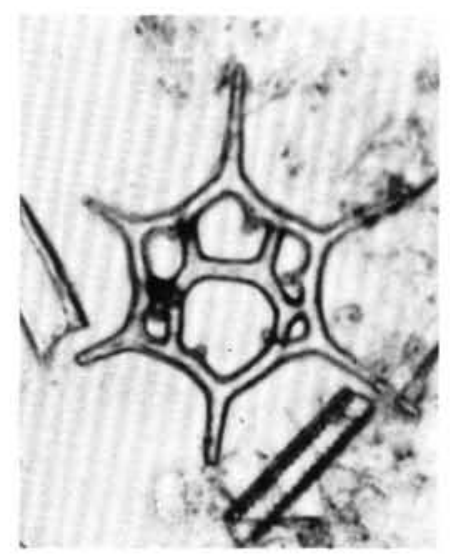

5

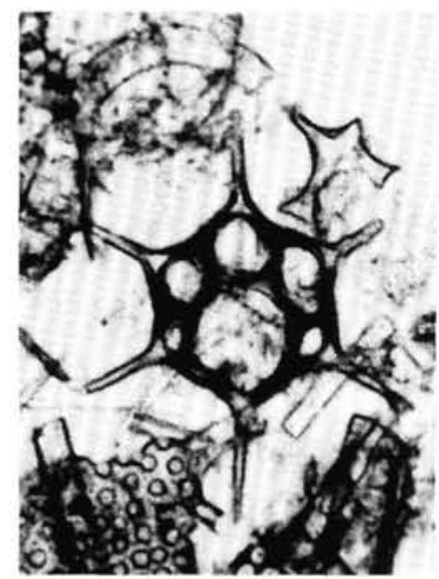

10

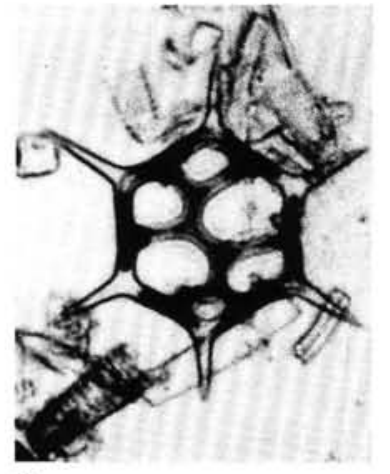

2

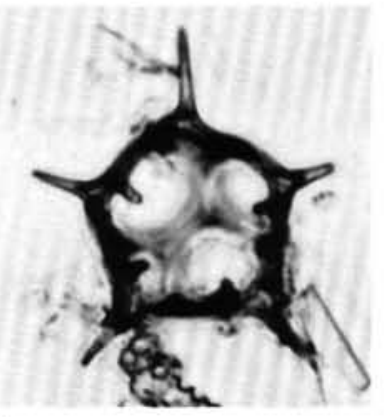

6

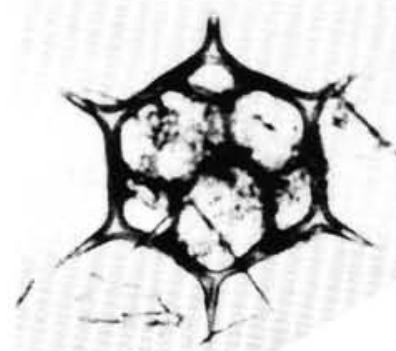

9

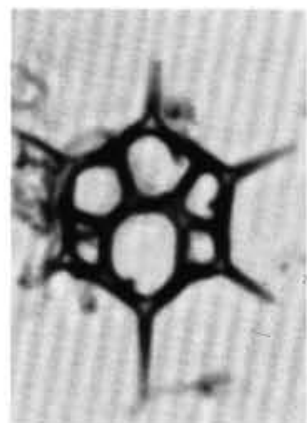

11

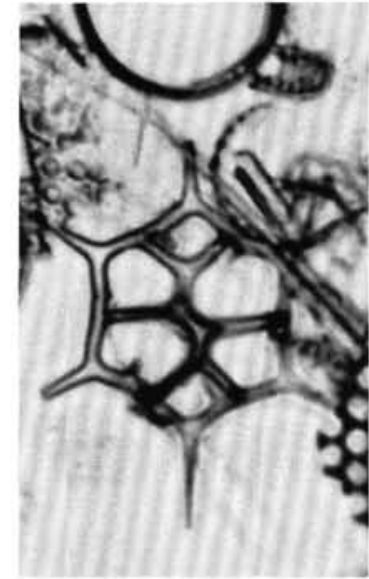

3

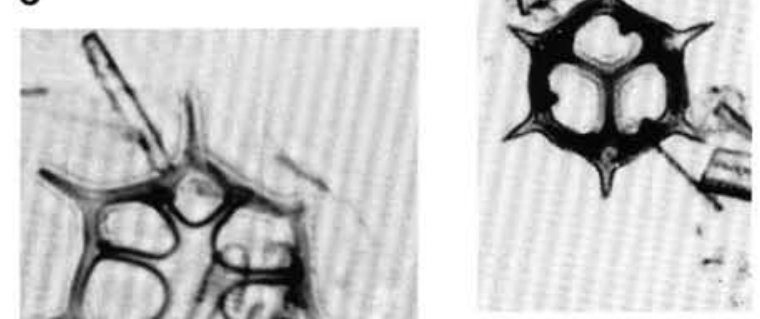

8

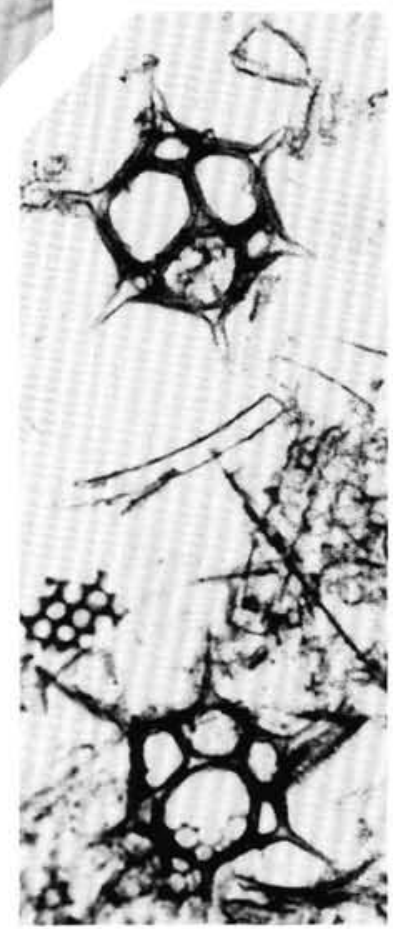

13

Plate 5. Specimens of the pseudofibula plexus of Distephanus speculum speculum from ODP Leg 113, 740 $\times$. 1-4. Distephanus speculum speculum f. pseudofibula. (1) Sample 113-690B-3H-5, 30-32; (2) Sample 113-689B-3H-5, 29-31 cm; (3) Sample 113-689B-3H-5, 29-31 cm; (4) Sample 113689B-3H-2, 30-32 cm. 5. Distephanus speculum speculum f. notabilis, Sample 113-689B-3H-2, 30-32 cm. 6. Distephanus speculum speculum f. pseudopentagonus $\mathrm{n}$. f., holotype, Sample 113-689B-2H-2, 33-35 cm. 7. Unusual and unnamed morphotype, Sample 113-689B-3H- 5, 29-31 cm. 8. Distephanus speculum speculum f. varians, Sample 693A-13R-2, 30-32 cm. 9. Distephanus speculum speculum f. varians, Sample 690B-3H-5, 30-32 cm. 10-12. Distephanus speculum speculum f. notabilis, (10) Sample 113-690B-3H-5, 30-32 cm; (11) Sample 113-689B-2H-5, 29-31 cm; (12) Sample 113-689B-3H-5, 29-31 cm. 13. Distephanus speculum speculum $\mathrm{f}$. varians (above) and Distephanus speculum speculum $\mathrm{f}$. notabilis (below), Sample 113-690B-3H-5, 30-32 cm. 


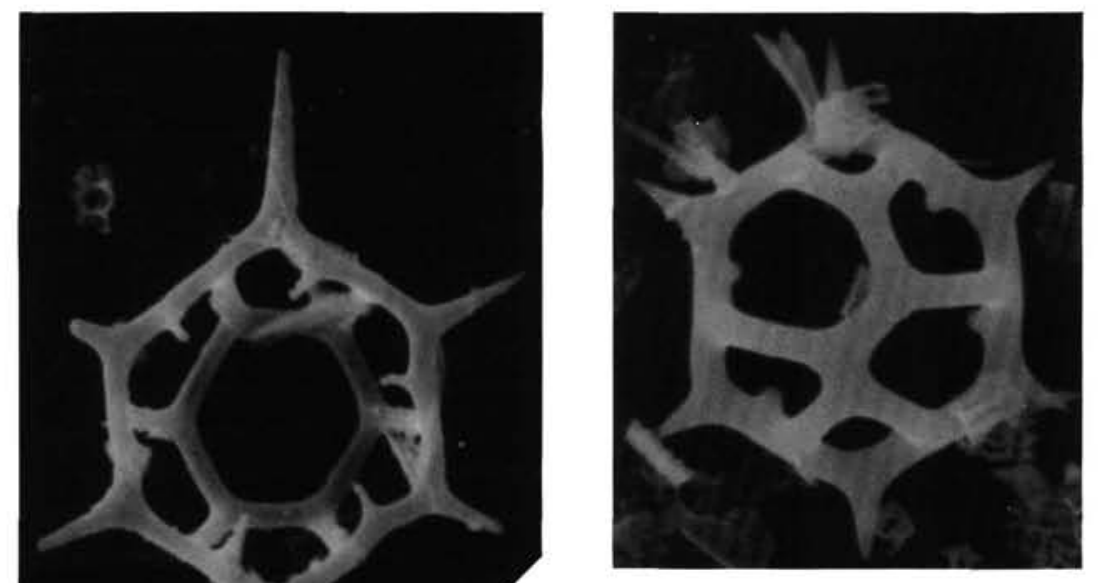

2
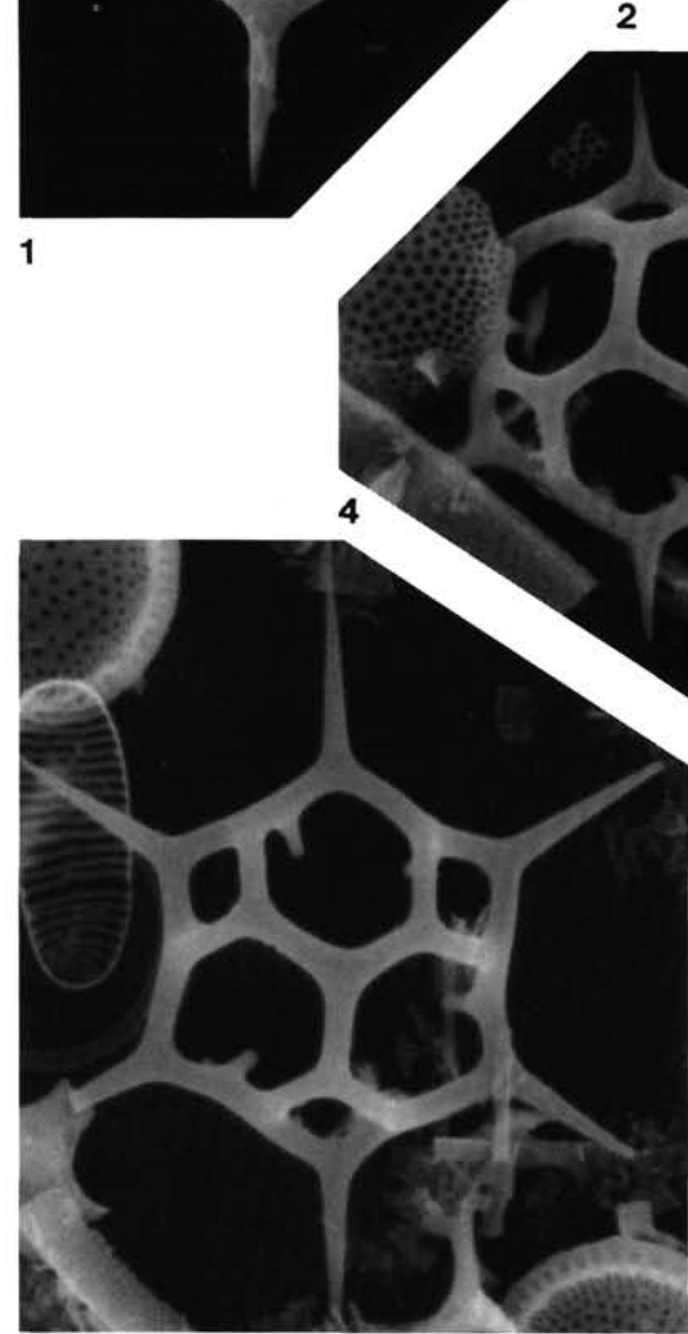

6

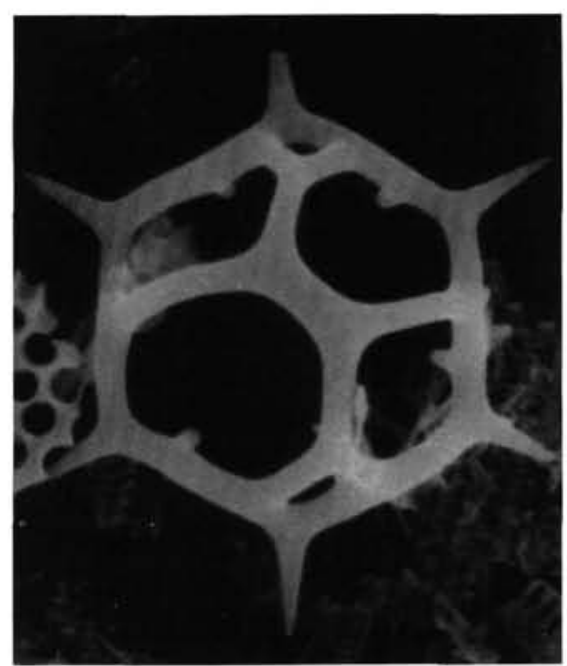

3

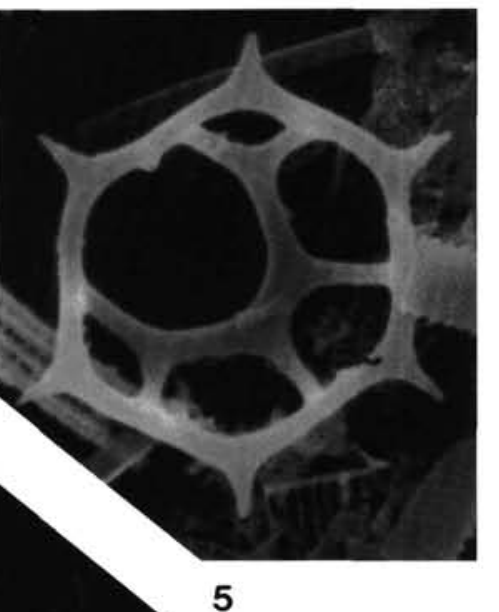

Plate 6. Scanning electron micrographs of specimens of the pseudofibula plexus from Leg 113,100 × all specimens except number 1 are from Sample 113-689B-3H-5, 29-31 cm. 1. Distephanus speculum speculum (with apical ring), Sample 113-689B-2H-5, 29-31 cm. 2, 3. Distephanus speculum speculum f. pseudofibula. 4. Distephanus speculum speculum f. varians. 5, 7. Distephanus speculum speculum f. notabilis. 6. Distephanus speculum speculum f. varians. 


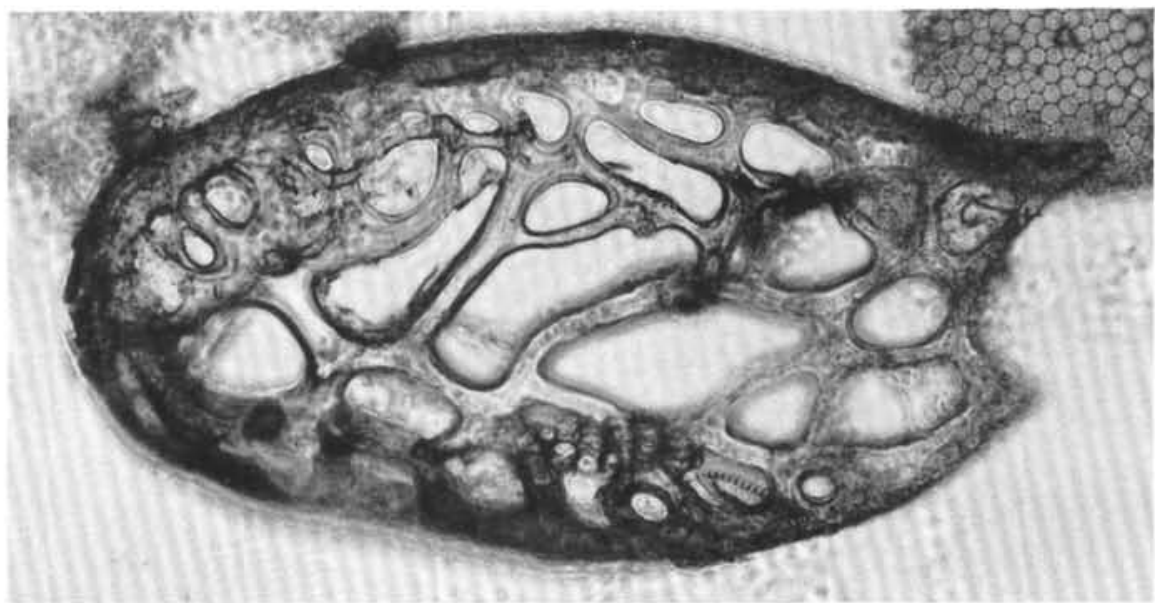

1

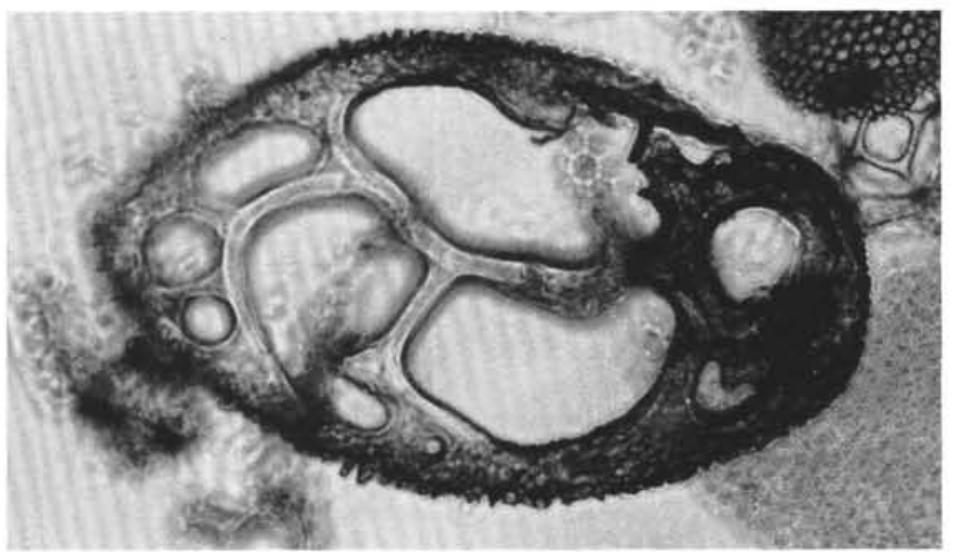

3

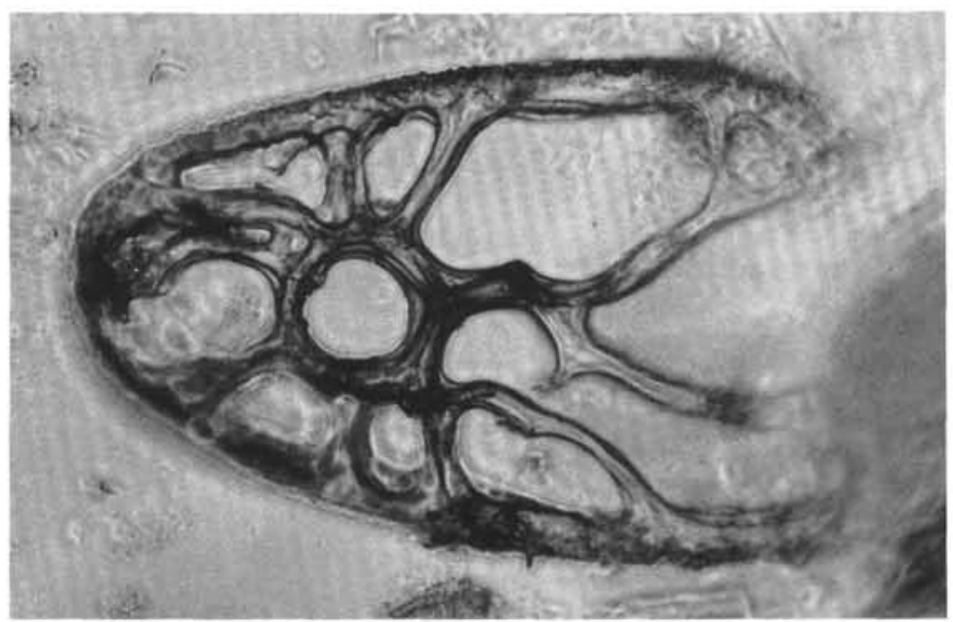

4

Plate 7. Ebridian specimens from ODP Leg 113, 435×. 1-3 Triskelion gorgon Gombos, Sample 113-689B-14H-5, 30-32 cm. 4. Adonnadonna primadonna Gombos, Sample 113-689B-14H-5, 30-32 cm. 5. Craniopsis octo Hovasse ex Frenguelli, Sample 113-689B-15H-2, $30-32 \mathrm{~cm}$. 6. Ebriopsis antiqua antiqua (Schulz), Sample 113-689B-14H-5, 30-32 cm. 7, 8. Ammodochium rectangulare (Schulz), (7) Sample 113-689B-14H-5, $30-32 \mathrm{~cm} ;(8)$ apical view, Sample 689B-15H-2, 30-32 cm.

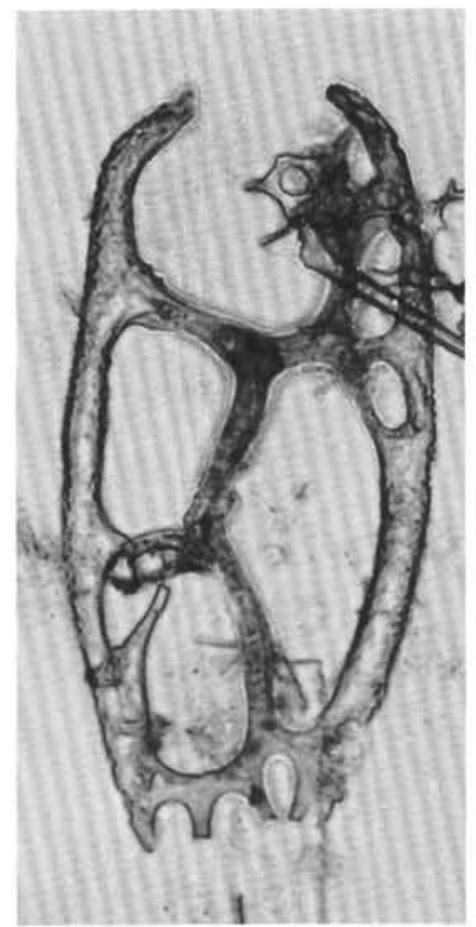

2

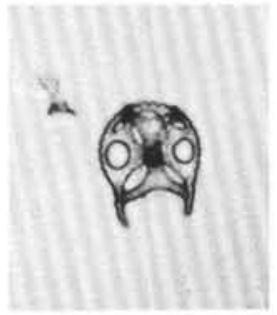

5

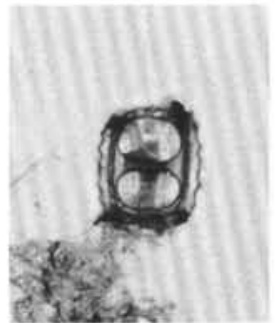

7

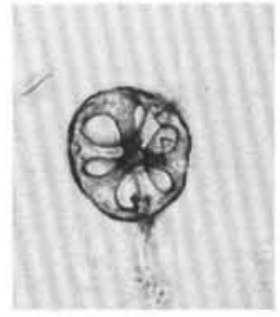

6

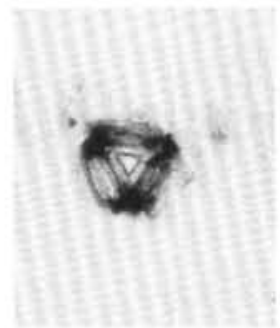

8 